\title{
18 Linguistic development studied in a general scientific corpus
}

\$1 Using the Corpus Corporum Latin language full-text collection, large amounts of data can now be processed automatically very quickly and reasonably correctly. Starting from what was described for scientific English in the previous chapter, this chapter tries to find similar characteristics of scientific Latin and to determine whether they have changed over time. At least to some extent, the development will also be considered from the other side: what features of scientific Greek were imported into Latin? As a a comparison set, some diachronic benchmark data for normal (non-scientific) Latin prose are produced first. For the scientific texts, forty of the Latin prose texts discussed in part 2 of this book from different fields and times were chosen. As a potentially interesting out-group, four translations from Greek are included, among them a non-scientific text, the Vulgate version of the New Testament. The idea is to first find characteristics that set all (or most) of the scientific texts apart from the benchmarks, and then to try to group them and to find different scientific Latin styles within them. For this, principal component analysis (PCA; see §6) will be used. This first relatively heterogeneous sample of forty texts was chosen in order to spot general trends; it will be compared to more homogeneous samples in chapter 20, which studies scientific Latin texts from some well-defined fields. Anyone familiar with such texts will know that different scientific styles or registers do exist in Latin, so one would expect them to be detectable quantitatively.

One might doubt whether much diachronic change will be detectable, given that the Latin language became fixed in the time of Cicero to such an extent that its written form stopped developing further according to normal language development and was petrified as a Hochsprache for the two thousand years to come (chap. 16 §1). Indeed, we can hardly expect scientific Latin to change in a clear manner through time. But as Latin did take up new words and constructions through these two millennia while retaining the older patterns, it is likely that new styles of writing emerged over time; these will be discussed in the next chapter (chap. 19). Besides, as Latin has a deep memory, it may be expected that old registers of scientific writing will be found to reappear again over time in some authors.

\section{Parts of speech}

The concept of PoS has, of course, its own long history (see chap. $9 \S 4$ above on Donatus, and in general Splett 2002) and is to a certain degree imposed on a language from outside. In any PoS system, there will always be cases that are hard to 
assign. The traditional system, which has been in use for Greek, Latin, and their descendent languages for more than two millennia, uses eight or nine parts of speech: noun (N), pronoun (PRON), adjective (ADJ), verb (V), adverb (ADV), conjunction (CONJ), preposition (PREP), interjection (INTER), and article (ART), the last of which is lacking in Latin. The Corpus Corporum Latin PoS tagger (TreeTagger, with language-specific data compiled by Gabriele Brandolini; http://www. cis.uni-muenchen.de/ schmid/tools/TreeTagger) uses this system and further distinguishes between some subtypes, e.g. among CONJ and PRON, verbal moods and the verb ESSE among V, and nominal cases among N. There are new approaches to defining PoS these days, designed to be applicable to all human languages. Universal PoS tags (UPOSTAG; http://universaldependencies.org/u/pos) is certainly the most successful such attempt. Table 3 compares the traditional system to UPOSTAG.

Table 3: Comparison between classical PoS and UPOSTAG.

\begin{tabular}{l|l|l|l|l|l|l|l|l|l|l|l|l|l}
\hline Classical & $\mathrm{N}$ & N:PR & PRON & ADJ & V & ESSE & ADV & CONJ & PREP & ART & INTER & NUM & \\
\hline UPOSTAG & NOUN & PROPN & PRON & ADJ & VERB & AUX & ADV & $\begin{array}{l}\text { SCONJ } \\
\text { CCONJ }\end{array}$ & ADP & DET & INTJ & NUM & PART \\
\hline
\end{tabular}

The rarer PoS italicised in table 3 (proper names, interjections, numerals) will not be used in our studies; among the rest, there is very little difference - basically only that UPOSTAG uses subordinating and coordinating CONJ as two different PoS and distinguishes particles from adverbs. Others have just received more general names (especially 'adpositions' instead of PREP). But, of course, the application of this general system to individual languages is still not always unambiguous. Thus, DET ('determiners') is wider than ART ('articles'), and it may be debated whether any words in Latin qualify as such (e.g. is, meus, omnis). Another major problem is that ADJ, PRON, N, and V sometimes overlap: for instance, omnis can behave as ADJ, PRON, DET, or even N. Another unclear case are participles (PTC): are they ADJ or V? The automated tagging is unable to differentiate between such difficult cases: it will, for instance, always assign PRON to omnis, ${ }^{1}$ and will treat lexicalised PTC as ADJ, others as V. ${ }^{2}$

In order to obtain a better understanding of precision and usability, the automated results of TreeTagger were evaluated by comparing them with the manual

1 This is clearly wrong in many instances. But it was not feasible to program a solution able to distinguish between cases.

2 Depending, obviously, on the lexicon used. In reality, cases such as the following should be differentiated: docta puella (ADJ) vs discipulus doctus est (V). Again, this could not be automated. 
PoS tagging done by the Laboratoire d'Analyse Statistique des Langues Anciennes (LASLA) ${ }^{3}$ for Cicero's De officiis. Corpus Corporum (CC) uses the Loeb edition digitised by Perseus as base text, LASLA the edition by Karl Atzert (Leipzig: Teubner, 1932). Slight differences are therefore to be expected simply because of the different base text: the Atzert edition has 386, or $1 \%$, more words than Loeb. ${ }^{4}$ Table 4 presents the numbers.

Table 4: Comparison between LASLA (manually determined) and CC (automated counting) for some features in Cicero's De officiis. The cases were counted among $\mathrm{N}$ only and are given as percentages of all $\mathrm{N}$.

\begin{tabular}{l|r|r|r|r|r|r|r|r|r|r|r}
\hline & ADJ & ADV & CONJ & N & PREP & PRON & V & $\begin{array}{r}\text { ABL/ } \\
\text { DAT }\end{array}$ & ACC & GEN & NOM \\
\hline LASLA & 2546 & 3117 & 4587 & 7530 & 2116 & 5129 & 8244 & $32.8 \%$ & $23.6 \%$ & $17.9 \%$ & $25.7 \%$ \\
\hline CC & 3453 & 3310 & 3538 & 7545 & 2381 & 4145 & 8299 & $32.3 \%$ & $24.8 \%$ & $18.3 \%$ & $24.6 \%$ \\
\hline Difference & $+36 \%$ & $+6 \%$ & $-23 \%$ & $+0 \%$ & $+13 \%$ & $-19 \%$ & $+1 \%$ & -0.4 & +1.2 & +0.4 & -1.2 \\
\hline
\end{tabular}

\begin{tabular}{l|r|r|r|r|r|r}
\hline & IND & SUB & INF & PTC & 1st SG & 3rd PAS \\
\hline LASLA & 3551 & 1777 & 1387 & 418 & 198 & 785 \\
\hline CC & 3559 & 1726 & 1299 & 1039 & 207 & 708 \\
\hline Difference & $+0 \%$ & $-3 \%$ & $-6 \%$ & $+148 \%$ & $+4 \%$ & $-10 \%$ \\
\hline
\end{tabular}

Among the cases, the rare vocative and locative were not considered, and the ablative and dative only in their combined sum (as CC distinguishes them very poorly). The figures for CC and LASLA correspond well for some of the values (N, $\mathrm{V}$, IND), but some differ conspicuously due to differences and sometimes mistakes in counting (marked in red in the table).

- There are different definitions for ADJ and PRON: CC includes e.g. alter, nullus, alius as ADJ (PRON in LASLA), besides some lexicalised PTC such as praesens. This accounts for the considerable differences. The numbers of PRON + ADJ together differ by only $1 \%$.

- LASLA counts the enclitics -que and -ve as separate CONJ. LASLA also counts autem, enim, and vero as CONJ:C. Subtracting these numbers (-que

3 The LASLA corpus data are freely available online (http://cipl93.philo.ulg.ac.be/OperaLatina/ users/MainInterface.aspx) after requesting log-in details. Their manual PoS tagging is based on the Forcellini lexicon. I thank LASLA, Université de Liège, for sharing their excellent data.

4 This is also partly to do with different definitions of words. For instance, filioque counts as two words for LASLA, as one for CC. 
108 , igitur 103, autem 266, vero 43, enim 269) renders the values at least closer $(-7 \%)$.

- The difference in PREP is largely due to the word cum, which CC wrongly always counts as PREP. 234 of the cums should be CONJ according to LASLA. This would leave a difference of $2 \%$. It would be very hard for software to tell the two cums apart.

- Participles (PTC) used as N (such as docti, adeptos) are counted as N in LASLA, as V:PTC or ADJ in CC. LASLA has 621 such cases; in the list, I subtracted them from LASLA's number for $\mathrm{N}$ and added them to the LASLA number for $\mathrm{V}$, which produced the practically identical numbers.

- The difference among the third person passives is due to the fact that CC cannot count synthetic forms such as factum est.

In conclusion, it seems that the results are reasonable for a fully automated counting and should be acceptable as a basis for the studies to come (although there is certainly room for improvement). As the same system was used for all texts, the results are at least consistent among themselves: the same errors will apply to all of them equally.

\section{Corpus approach}

\$2 As the main basis of comparison, five large Latin ad hoc prose corpora were generated for five important time periods of Latin. The corresponding texts were loaded as TEI xml files into Corpus Corporum (CC), where automatic part of speech (PoS) tagging was performed using TreeTagger, and the resulting data downloaded again from the server as PoS-tagged TEI xml files which could be studied further with linguistic Unix tools (sed, grep, and the like). The tagging produces data of the form: $<$ w type="PRON:DEM" lemma="hic" $>$ his $</$ w $>$ for each word in the text, thus assigning each word (here his) its lemma and a type including its PoS. If the lemma in question is not known to the database, the type will be unreliable, for instance $<\mathrm{w}$ type="ADJ" lemma="unknown" $>$ Chananaea $</ \mathrm{w}>$ (type should be N:nom, not ADJ). 'Unknown' words are mostly proper names; they were removed from the samples for our studies. The entire process was based exclusively on open-source software.

Basic data about these ad hoc corpora is presented in table $5 .^{5}$ Additionally, some specific, non-scientific corpora were compiled in order to permit compari-

5 All texts are in Corpus Corporum, where further information about them, including references, can be found. These ad hoc corpora contain all prose texts from the respective corpora and time ranges (in 2018); any lines of verse in corpora 1-5 were removed. Only texts of at least 1,000 words in length were included. The author's death date or floruit (as available in CC) was used as an ap- 
son with potentially interesting other registers. A large corpus of metrical texts (mostly hexametric), and smaller ones of the Digesta (see chap. 8 §3), a small collection of charters from the High Middle Ages, and the New Testament Vulgate (as a non-scientific text that was translated from the Greek) were used. It became clear above how important jurisprudence was for the development of Latin as a language of science, so the Digesta may reveal interesting traits. The Vulgate is included for its great importance for Christian Latin in general (see chap. 9 §2). The language of scientific poetry will be compared below, as will that of historiography, whose language may exhibit similarities with charters (both in chap. 20). The language of these additional text collections differs greatly from that of the diachronic prose texts.

Table 5: Information on the five benchmark samples and out-groups to be used below.

\begin{tabular}{|c|c|c|c|c|c|c|c|}
\hline & Name & Source & Time range & Authors & Texts & Million words & Avg word length ${ }^{6}$ \\
\hline 1 & Classical Latin & $\begin{array}{l}\text { Perseus } \\
\text { collection }\end{array}$ & 100 BC-AD 200 & 15 & 57 & 2.215 & 6.38 \\
\hline 2 & Late Antiquity & PL & $200-450$ & 57 & 430 & 11.102 & 5.63 \\
\hline 3 & $\begin{array}{l}\text { Carolingian } \\
\text { times }\end{array}$ & PL & $780-900$ & 78 & 331 & 10.447 & 5.75 \\
\hline 4 & Twelfth century & PL & $1100-1220$ & 162 & 738 & 26.182 & 5.77 \\
\hline 5 & Early modern & $\begin{array}{l}\text { CroaLA } \\
\text { (Croatian authors) }\end{array}$ & $1500-1820$ & 40 & 50 & 1.877 & 6.21 \\
\hline 6 & Poetry & $\begin{array}{l}\text { Perseus collec- } \\
\text { tion and others }{ }^{7}\end{array}$ & $\begin{array}{l}50 \mathrm{BC}-\mathrm{AD} \\
1793^{8}\end{array}$ & 162 & 593 & 3.188 & 5.49 \\
\hline 7 & Roman law & Digesta ${ }^{9}$ & 1st-6th century & 38 & 9,139 & 0.840 & 5.72 \\
\hline
\end{tabular}

proximation to date the texts. Obviously, undated texts were not used (CC currently has date tags for only some $85 \%$ of its texts).

6 The average of the five prose samples is $5.95 \pm 0.32$ letters. Mediaeval and poetic texts seem to have a shorter average word length than classical and early modern ones.

7 Comprising the texts in corpus 15 'Poetica' on CC, plus Vergil and Ovid. The longest texts are (in descending order): Michael Hospitalis, Carmina; Carmina Burana; Marcello Palingenio Stellato, Zodiacus vitae; Ugolino Verino, Carlias; Francesco Petrarca, Africa; Rafael Landívar, Rusticatio mexicana. Many of these texts were written by Italian humanists.

8 Metrical Latin poetry is very conservative, so it was decided to bring texts from different times together. We will compare the numbers to numbers obtained for Vergil and Ovid only. They are surprisingly similar.

9 The Digesta were collected by order of Justinian in 530-533. 9,139 is the number of excerpts, 38 is the number of authors cited in the Florentinus list. See chap. $8 \S 12$ above. 
Table 5: (continued)

\begin{tabular}{l|l|l|l|r|r|r|r}
\hline 8 & Charters & $\begin{array}{l}\text { Arezzo (ed. in } \\
\text { Pasqui 1899-1937) }\end{array}$ & 1130-1222 & $\mathrm{n} / \mathrm{a}$ & 115 & 0.081 & 5.81 \\
\hline 9 & Bible & $\begin{array}{l}\text { Vulgate, Novum } \\
\text { testamentum }^{11}\end{array}$ & ca. 400 & $\mathrm{n} / \mathrm{a}$ & 1 & 0.127 & 5.32 \\
\hline
\end{tabular}

Parameters that may be important for scientific language were determined for the five large benchmark samples: distribution of PoS, moods, cases, suffixes, and subordinate clauses. To begin, the PoS distribution was calculated for each text within the five large diachronic samples, then values were averaged per group. This made it possible to determine standard deviations within samples, which indicate how much variation there is. The PoS distribution has changed only little over time (table 6 and fig. 31) - mostly not beyond the standard deviation range for the classical sample. The amount of verbs employed diminished somewhat through this timespan, but the amount of nominal PoS (defined as N + ADJ + PRON) rises perceptibly only in the early modern period - thus, Mediaeval Latin can hardly be said to become more 'nominal', even though the numbers of $\mathrm{N}$ do rise somewhat. Only the numbers of PREP rise after the first sample to a value beyond 1 stdev of the deviation within that sample. This is the only PoS consistently outside the standard deviation ranges. ADJ become much more frequent in early modern times, $\mathrm{N}$ become slightly more common with time, while $\mathrm{V}$ and PRON become less so. Averaging the PoS values for the five samples shows that the classical sample differs most strongly from the average and thus from the (mostly Christian) rest.

Table 6: Average and standard deviation for PoS over all texts in the five ad hoc prose samples as percentages of the total.Values more than $1 \mathrm{stdev}$ outside the antique value are highlighted in colour: ${ }^{12}$ red means less than the antique average minus 1 stdev, green more than the average plus 1 stdev

\begin{tabular}{l|r|r|r|r|r|r|r|r|r|r}
\hline & $\begin{array}{r}100 \mathrm{BC}- \\
\mathrm{AD} 200\end{array}$ & stdev & $200-450$ & stdev & $780-900$ & stdev & $1100-1220$ & stdev & $1500-1820$ & stdev \\
\hline ADJ & 11.12 & 1.54 & 9.67 & 3.97 & 10.79 & 2.42 & 10.52 & 2.52 & 13.95 & 3.00 \\
\hline ADV & 9.22 & 1.10 & 8.61 & 1.98 & 7.90 & 1.59 & 8.66 & 1.96 & 8.79 & 1.78 \\
\hline
\end{tabular}

10 Digitised by the ALIM project, http://it.alim.unisi.it. The non-standardised spelling complicates the automatic tagging, and the quality of the results will be worse than usual.

11 Ed. Tweedale; see chap. 8 §2 above.

12 The values add up to only $98 \%-99 \%$ of all words because TreeTagger also tags the few abbreviations, interjections, and Roman numerals in the text. 
Table 6: (continued)

\begin{tabular}{l|r|r|r|r|r|r|r|r|r|r}
\hline CONJ & 9.34 & 1.56 & 10.37 & 1.65 & 9.84 & 1.98 & 9.42 & 1.47 & 8.27 & 1.88 \\
\hline $\mathrm{N}$ & 23.34 & 3.84 & 25.19 & 4.31 & 26.90 & 4.02 & 26.16 & 4.01 & 25.42 & 2.98 \\
\hline PREP & 6.73 & 1.04 & 7.82 & 1.48 & 8.56 & 1.14 & 8.50 & 1.27 & 7.87 & 1.87 \\
\hline PRON & 15.23 & 2.97 & 13.93 & 2.62 & 13.01 & 2.28 & 13.28 & 2.24 & 12.36 & 2.44 \\
\hline $\mathrm{V}$ & 23.93 & 2.12 & 23.12 & 2.94 & 21.67 & 2.43 & 21.91 & 2.43 & 21.77 & 2.30 \\
\hline $\mathrm{N}+\mathrm{ADJ}+$ & 49.69 & 2.51 & 48.80 & 4.82 & 50.71 & 4.11 & 49.96 & 4.13 & 51.73 & 2.93 \\
PRON & & & & & & & & & & \\
\hline
\end{tabular}

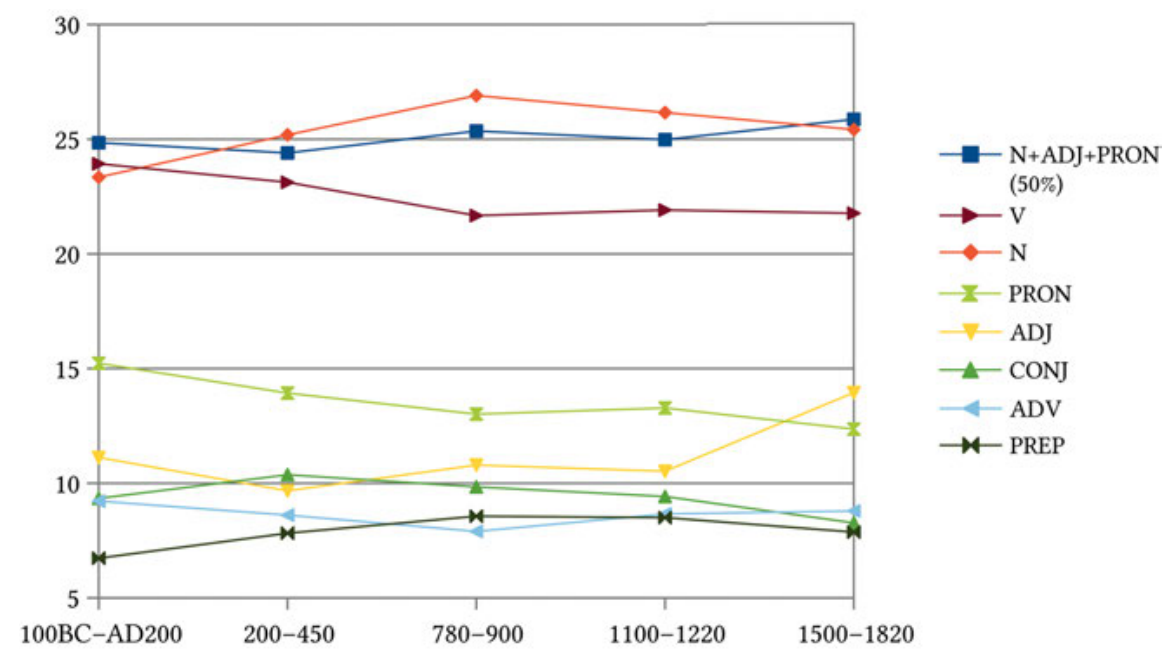

Fig. 31: PoS distribution over time. The $\mathrm{N}+\mathrm{ADJ}+\mathrm{PRON}$ value was halved to fit the scale. Values on the $y$-axis in percent.

The PoS values provide a first impression of how much (or rather, how little) variability is to be expected in Latin over the centuries. A second table (table 8 below) will compare the cases, the most important moods of the verb, and some other specific features that may be revealing for scientific language. Above (chap. 17), it was observed that scientific English tends to favour passive constructions and noun phrases. The latter correspond mostly to compounds in Greek and German (see chap. 9 §5), but in Latin they are often expressed by relative clauses. Therefore, the relative pronoun qui (REL) and the verb esse, which is often involved in such constructions, were also counted. In the wake of Greek usage, sentence-modifying or discourse particles may be another feature typical 
of scientific Latin: ${ }^{13}$ at, autem, enim, ergo, igitur, nam, and vero were counted. Kroon, who studied these particles in great detail, categorises them in a table (see table 7). ${ }^{14}$

Table 7: Latin sentence-modifying particles, from Kroon (1995: 373).

\begin{tabular}{llll}
\hline & $\begin{array}{l}\text { Presentational } \\
\text { 'connective' }\end{array}$ & $\begin{array}{l}\text { Interactional } \\
\text { 'situating' }\end{array}$ & $\begin{array}{l}\text { Interactional } \\
\text { 'connective' }\end{array}$ \\
\hline Causal/consecutive & nam, igitur & enim & ergo \\
\hline Adversative & autem & vero & at \\
\hline
\end{tabular}

Another feature of some kinds of Latin often criticised by humanists (chap. 12 §2) is the use of suffixation, which will be discussed below as a surrogate for compounding (chap. 24 §7). In order to obtain numbers, a sample of seven especially common nominal suffixes (-tio/-sio, -tas, -itia, -ntia, -mentum, -tor/-sor, -tudo) and eight adjectival ones (-alis/-aris, -bilis, -eus, -icus, -inus, -ivus, -orius, -osus) was used. Promising suffixes for this purpose had already been selected in Roelli (2013: 334-335, based on Leumann 1944). ${ }^{15}$ The suffixes' rôles can be briefly described as follows. For the nominal suffixes:

- -tio/-sio: expresses a process (e.g. actio);

- -tas, -ia (including -itia, -ntia), -tudo: express a quality (e.g. gravitas);

- -mentum: expresses a tool or the means to achieve something (e.g. monumentum);

- $\quad$ tor/-sor: expresses the agent (e.g. actor). For the adjective suffixes:

- -ivus, -orius (added to verb roots): assert that something possesses the verbal action inherently (e.g. activus);

- $\quad$-bilis (added to verb roots): expresses the possibility of undergoing the verbal action (e.g. habitabilis);

- -osus, -eus (added to nominal roots): express what something is endowed with, or what it consists of (e.g. gratiosus);

13 Ramshorn (1842) presents larger groups of such modifiers. Adversative particles: sed, verum, vero, at, atqui, autem; explanatory ones: nam, namque, enim, etenim; concluding ones: itaque, igitur, ergo, eo, ideo, idcirco, propterea, (pro)inde, quare, quamobrem, quapropter. I used only the seven most important ones, those studied by Kroon (1995).

14 Some caveats in Langslow (2000b: 559), especially for medical Latin.

15 Helander (2014: 47) studies a similar list for the adjective suffixes. 
- $\quad$-icus, -alis/-aris, -inus (added to nominal roots): express a relationship (e.g. pluvialis). ${ }^{16}$

The use of these suffixes has, on the whole, remained constant in Latin. Only very rarely did special, new constructions for some of them arise in Post-Classical Latin. For instance, the suffix -ivus plus objective genitive is often used in the High Middle Ages in a special syntagm (e.g. novum testamentum est veteris impletivum). ${ }^{17}$ There are also suffixes derived from roots, such as -ficus, -formis, -oides, that become frequent in early modern times. It is obvious that a set of such suffixes that can enhance language systematically is very useful for scientific expression. In fact, most of these suffixes are still very common in scientific English today.

Ablativi absoluti may be another useful feature for scientists, as they make it possible to state the circumstances of the main action in a condensed way. An attempt was made to estimate their numbers automatically. Of course, counting this special feature of the Latin language automatically is not a trivial matter; in some cases it even remains unclear to Latinists whether an ablative construction is to be read as an ablativus absolutus or as another kind of ablative (especially an instrumental one). My tentative approach was to count all occurrences of two ablative forms with a maximum of two other words in between and no strong punctuation, where one of the two was a participle. Checking a small sample shows that most ablativi absoluti were indeed found, but of course there were also some false positives. A comparison with manually determined values by LASLA shows that our amounts tend to represent between $80 \%$ and $150 \%$ of the true value. However, as the determined numbers can, for instance, differ by a factor of ten between Pliny and Anselm of Canterbury, the numbers may still be of some relative value. The numbers are included here in the knowledge that they are not very accurate. ${ }^{18}$ Late Antiquity has lower numbers than the rest, which have rather similar average numbers, although stdevs are large, indicating that authors differ considerably in their frequency of employing the ablativus absolutus.

The last feature in the table is textual entropy. ${ }^{19}$ As in entropy defined in physics, the degree of orderliness of a text string is measured. Whereas very orderly

16 Words with these suffixes were counted using the Linux grep tool; they were required to be the correct PoS and have at least two more letters (thus excluding words such as divus, vivus, binus, etc.). For -icus, words in -ficus (compounds with facere) were excluded. Some false positives, such as semivivus, had to remain in the count and will distort the result slightly.

17 As Stotz (1996-2004: VI, §86 = vol. 2, p. 356) points out.

18 I thank Alexandra Bünzli for writing the perl script I used.

19 Proposed by Shannon (1951), then used by Evrard (1967: 81-85), this number is calculated as the negative sum $-\Sigma\left[\mathrm{fj} \times \log _{2}(\mathrm{fj})\right]$ over all lemmata $\mathrm{j}$, where $\mathrm{fj}$ is the relative frequency of lemma 
sequences of strings are at best very uninteresting and predictable texts, say est est est est, real and interesting texts are less predictable and monotonous. The entropy value is a measure that indicates the unpredictability of words in a text, the 'news value' of the next word in the sequence; it is measured in bits of information per word. Values tend to range between 7 and 11 bits. The entropy value rises slightly with more tokens; for texts with too few tokens, the value depends significantly on the text's length and therefore makes little sense. Bentz et al. (2017:14) feel that the problems are not present above 50,000 tokens. The values below were calculated only for texts longer than 10,000 words. ${ }^{20}$ The graphic in figure 32 makes this choice plausible.

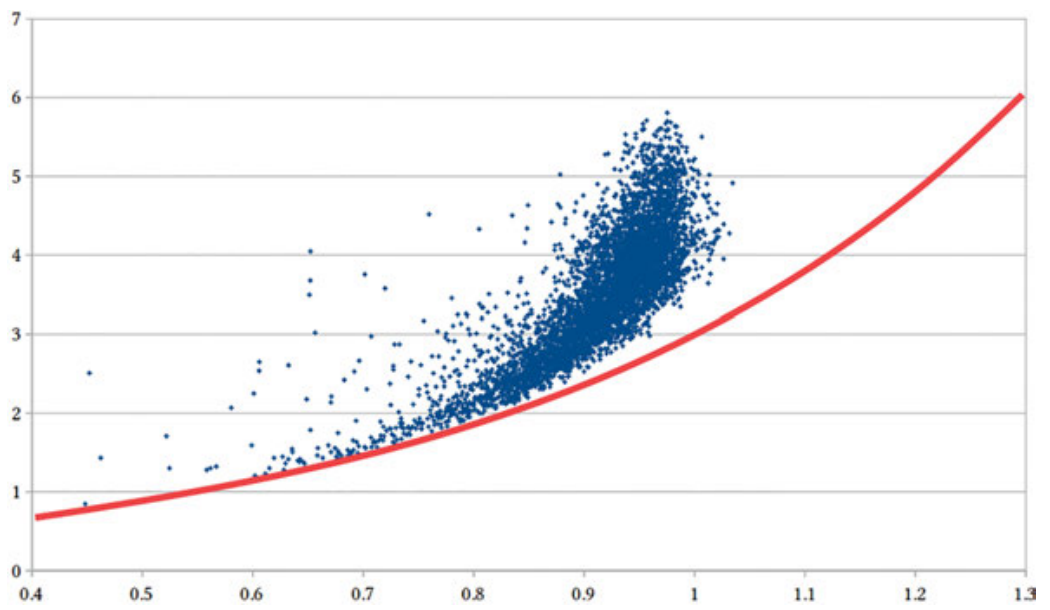

Fig. 32: Doubly logarithmic $\left(\log _{10}\right)$ plot of entropy values for all texts in PL: the $x$-axis shows the entropy value, the $y$-axis the number of tokens (words). The red curve shows the maximum possible entropy value ( $\log _{2}$ of the number of tokens), representing the case that each token is used exactly once. Above $y$-value 4 (10,000 tokens), the real values no longer seem to follow the maximum possible value closely.

The first thing that hits the eye in table 8 is that the standard deviations for all moods and cases are relatively high: different authors differ strongly, even within the same period. Nonetheless, there are some parameters that deviate on average more than 1 stdev from the classical value (again, highlighted in colour). The

j. The same formula is used to calculate entropy in information theory, hence the name. More precisely, this is uni-gram entropy, as defined in Bentz et al. (2017: 5, formula 3).

20 For the five corpora in ascending chronological order, this means 40, 242, 161, 375, and 18 texts respectively. 
amounts of IND rise significantly in the Middle Ages and drop even more afterwards, when humanists overcompensate in employing this un-classical usage. SUB exhibit the inverse behaviour. INF drop in the Middle Ages while PTC rise. The former change is very conspicuous, such that lower numbers of infinitives might be a typical characteristic of Mediaeval Latin. As ACC numbers also drop somewhat, this may be connected with the rarer use of the accusativus cum infinitivo. Interestingly, the humanists have somewhat higher numbers of INF again, but use PTC even more than Antiquity. This may be an attempt to imitate Greek, a language very fond of participle constructions. In the Middle Ages, the high PTC numbers may point to influence from biblical Latin (although the value for our NT Vulgate sample is only slightly higher than the benchmarks).

Table 8: Some more specific, potential characteristics for scientific language. The verbal forms are given as percentages of all $\mathrm{V}$, the nominal ones as percentages of all $\mathrm{N}$. For details, see the main text.

\begin{tabular}{|c|c|c|c|c|c|c|c|c|c|c|}
\hline & $\begin{array}{r}100 \mathrm{BC}- \\
\mathrm{AD} 200\end{array}$ & stdev & $200-450$ & stdev & $780-900$ & stdev & $1100-1220$ & stdev & $1500-1820$ & stdev \\
\hline IND ( $\%$ of $V)$ & 45.60 & 4.70 & 50.40 & 7.39 & 47.49 & 8.60 & 50.88 & 7.86 & 42.64 & 7.30 \\
\hline SUB (\% of V) & 16.71 & 3.26 & 14.90 & 3.71 & 14.40 & 5.47 & 13.58 & 5.82 & 14.87 & 4.52 \\
\hline INF (\% of V) & 15.07 & 2.92 & 11.50 & 3.96 & 11.34 & 3.29 & 11.04 & 3.61 & 12.71 & 3.51 \\
\hline PTC (\% of V) & 17.52 & 5.24 & 18.03 & 5.31 & 21.25 & 7.04 & 19.38 & 6.22 & 23.50 & 5.42 \\
\hline NOM (\% of N) & 26.52 & 3.57 & 30.02 & 6.06 & 27.12 & 4.66 & 28.36 & 4.66 & 25.49 & 4.55 \\
\hline GEN (\% of $N)$ & 12.46 & 1.97 & 14.93 & 2.97 & 16.78 & 3.06 & 16.32 & 3.06 & 15.74 & 2.76 \\
\hline$A C C(\%$ of $N)$ & 27.42 & 4.00 & 26.58 & 3.68 & 24.10 & 3.80 & 24.56 & 3.55 & 26.28 & 3.65 \\
\hline $\mathrm{ABL} / \mathrm{DAT}(\%$ of $\mathrm{N})$ & 33.60 & 3.43 & 28.47 & 4.68 & 32.00 & 4.70 & 30.76 & 4.71 & 32.48 & 4.5 \\
\hline ESSE (\%) & 3.80 & 0.99 & 3.82 & 1.26 & 3.15 & 1.15 & 3.29 & 1.31 & 2.63 & 0.95 \\
\hline REL (\%) & 3.98 & 0.75 & 3.68 & 0.79 & 3.38 & 0.73 & 3.49 & 0.71 & 2.96 & 0.83 \\
\hline CONJ:S (\%) & 2.86 & 0.69 & 3.20 & 0.92 & 2.91 & 0.86 & 2.88 & 0.79 & 2.29 & 0.61 \\
\hline 1st SG (\% of V) & 6.79 & 4.29 & 4.26 & 3.42 & 3.10 & 2.77 & 3.38 & 2.53 & 5.43 & 5.00 \\
\hline 3rd PAS ( $\%$ of $V$ ) & 6.38 & 2.43 & 8.05 & 2.67 & 8.41 & 3.16 & 9.01 & 3.80 & 6.09 & 2.68 \\
\hline ADJ-SUF (\%) & 1.29 & 0.40 & 1.14 & 0.47 & 1.57 & 0.73 & 1.51 & 0.57 & 1.52 & 0.69 \\
\hline N-SUF (\%) & 3.80 & 1.02 & 4.19 & 1.25 & 4.86 & 1.16 & 5.03 & 1.42 & 4.02 & 1.48 \\
\hline Modifiers (\%) & 1.42 & 0.62 & 1.78 & 0.75 & 1.28 & 0.56 & 1.40 & 0.59 & 1.02 & 0.49 \\
\hline $\begin{array}{l}\text { ABL ABS per } \\
1,000 \text { words }\end{array}$ & 3.45 & 2.48 & 2.56 & 1.80 & 3.77 & 2.68 & 3.17 & 2.30 & 3.95 & 1.82 \\
\hline Entropy & 9.36 & 0.50 & 8.83 & 0.53 & 9.11 & 0.42 & 9.16 & 0.47 & 9.27 & 0.61 \\
\hline
\end{tabular}


The use of cases remains relatively stable for NOM and ACC, except that the sample from Late Antiquity exhibits a rather high percentage of NOM and a correspondingly low number of ABL/DAT, possibly because these cases were becoming rarer in the still-spoken language. The number rises again from Carolingian times onward (when grammar was learned in school). Most interesting for scientific language may be GEN, which is the only case that becomes more than 1 stdev more common as time passes, and even remains so in the humanist sample. This may be one of the reasons why Mediaeval Latin gives the impression of being more 'nominal', ${ }^{21}$ as 'nominal' GEN constructions contrast with 'verbal' ACC constructions. Unexpectedly, both ESSE and the REL pronoun become rarer as time passes, although strongly so only in the humanist sample. Constructions with relative clauses and a high concentration of the copula will have been perceived as 'scholastic' and worth avoiding, although it can now be seen that they were even more common in classical times. The third person passive (singular and plural) is somewhat more common in the Middle Ages than before or after, but it is the first person singular that surprises by being a lot less common in the Middle Ages. Interestingly, the numbers of the third person passive are highest for the twelfthcentury sample, at the beginning of scholastic writing. Nominal suffixes are clearly another feature that was perceived as 'scholastic' and avoided in the humanist sample, though adjective suffixes apparently were not.

More detailed data about these suffixes are shown in table 9 and figure 33. Because their occurrences are usually low in number, and chance fluctuations are thus to be expected, averages across all texts were used (thus, no longer counting per text then averaging the numbers obtained for each text in one corpus), except for the first corpus (Antiquity) in order to still have a stdev value. The first and third rows in table 9 thus reflect the difference one will generally have to expect between these two ways of counting. It is mostly small (very small for the PoS in table 6 above, numbers not printed); only -bilis, -ivus, -osus; -itia, -ntia exhibit a difference of more than $10 \%$, indicating that their frequencies were especially variable among antique authors and texts. Standard deviations are in general high; only -alis and -tio consistently reach values more than 1 stdev higher than in the classical samples in the Middle Ages and early modern times. But, presumably due to criticism by humanists, some frequencies drop significantly in early modern times compared to the Middle Ages, especially for -tas, -ntia, -bilis - most abruptly for the first. ${ }^{22}$ Some others, which were apparently not consciously asso-

21 See chap. 11 §2 above: Stotz on scholasticism’s ‘ausgesprochenen Nominalstil' ('pronounced nominal style').

22 This suffix was especially targeted by the humanists, e.g. Lorenzo Valla, quoted in chap. $12 \S 2$ above. 
ciated with 'scholasticism', such as -tio, -alis, keep rising through the entire sample period; a few (-eus, -ivus) rise decidedly in early modern times; and some remain quite constant (-mentum, -osus, -tudo, -itia). ${ }^{23}$

Table 9: Occurrences of suffixes in \%o of words. The first row was measured per text and then averaged; the second row contains the stdev over all texts in the same corpus. All further rows are simple averages over all texts in the respective corpus. Thus, the second 100 BC-AD 200 row is the one that should be compared to the numbers from the other corpora.

\begin{tabular}{|c|c|c|c|c|c|c|c|c|c|c|c|c|c|c|c|}
\hline & $\begin{array}{l}\text {-alis/ } \\
\text {-aris }\end{array}$ & -bilis & -eus & -icus & -inus & -ivus & -orius & -osus & -itia & $\begin{array}{r}- \text { men- } \\
\text { tum }\end{array}$ & -ntia & -tas & -tio & -tor & -tudo \\
\hline $\begin{array}{l}100 \mathrm{BC}- \\
A D 200\end{array}$ & 2.24 & 1.29 & 0.75 & 3.30 & 1.12 & 0.32 & 0.26 & 1.89 & 1.76 & 1.88 & 4.42 & 10.73 & 9.69 & 4.08 & 2.26 \\
\hline stdev & 0.81 & 0.73 & 0.81 & 2.05 & 0.71 & 0.23 & 0.24 & 1.04 & 1.30 & 1.00 & 2.08 & 3.76 & 4.97 & 2.42 & 1.62 \\
\hline $\begin{array}{l}100 B C- \\
A D 200\end{array}$ & 2.24 & 1.08 & 0.81 & 3.08 & 1.10 & 0.28 & 0.28 & 1.61 & 1.41 & 1.85 & 4.01 & 10.10 & 10.20 & 4.24 & 2.26 \\
\hline $\begin{array}{l}200- \\
450\end{array}$ & 2.94 & 1.69 & 0.93 & 1.99 & 1.30 & 0.19 & 0.09 & 1.10 & 1.99 & 2.03 & 4.97 & 11.78 & 12.76 & 3.66 & 1.90 \\
\hline $\begin{array}{l}780- \\
900\end{array}$ & 3.61 & 2.08 & 1.08 & 2.93 & 1.98 & 0.44 & 0.33 & 1.06 & 1.91 & 2.07 & 5.51 & 12.78 & 16.08 & 4.66 & 2.12 \\
\hline $\begin{array}{l}1100- \\
1220\end{array}$ & 4.08 & 2.09 & 1.05 & 3.09 & 1.58 & 0.37 & 0.33 & 1.26 & 2.06 & 2.01 & 6.05 & 13.40 & 17.18 & 3.86 & 2.37 \\
\hline $\begin{array}{l}1500- \\
1820\end{array}$ & 4.30 & 1.02 & 1.47 & 2.93 & 1.25 & 0.70 & 0.24 & 1.01 & 1.85 & 2.14 & 4.65 & 9.10 & 16.64 & 3.64 & 1.82 \\
\hline avg & 3.44 & 1.59 & 1.07 & 2.81 & 1.44 & 0.40 & 0.25 & 1.21 & 1.85 & 2.02 & 5.04 & 11.44 & 14.56 & 4.01 & 2.09 \\
\hline stdev & 0.85 & 0.52 & 0.25 & 0.46 & 0.35 & 0.19 & 0.10 & 0.24 & 0.25 & 0.11 & 0.78 & 1.80 & 3.00 & 0.43 & 0.23 \\
\hline
\end{tabular}

Finally, a closer look is taken at subordination, which seems to be of special importance for the well-structured, syntactic connection of scientific thoughts. Total numbers are counted in table 10 below. ${ }^{24}$ The general numbers above (table 6) have shown that numbers of subordinating CONJ remain quite constant, before dropping significantly in the early modern sample. A selection of eight CONJ:S

23 These results are comparable to those in Roelli (2013), which were acquired from a much smaller sample.

24 The common ones in the benchmark samples are antequam, cum/quum, donec, dum, dummodo, etiamsi, etsi, igitur, ne, neve, ni, nisi, nonnisi, posteaquam, postquam, priusquam, prout, quam, quamquam, quamvis, quando, quandoquidem, quanquam, quasi, quia, quo, quod, quominus, quoniam, si, sin, siquidem, tametsi, tamquam, tanquam, ut. 
and two conspicuous PREP, sometimes in semantic groups, was counted to this end (qui was already included in table 8 above as REL).

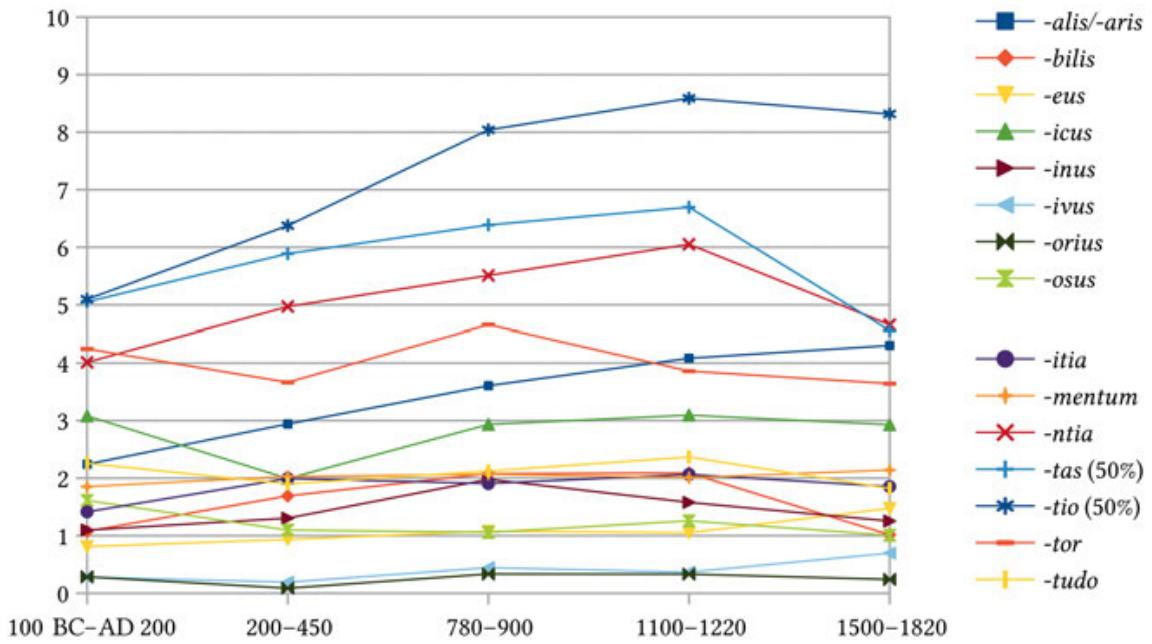

Fig. 33: Suffixes in the five samples in \%o. The values of the two most common nominal suffixes, -tas and -tio, have been halved to fit the scale.

Table 10: Numbers for some subordinating CONJ in \%o. For the differences between the first and third rows, see the comments on table 9 .

\begin{tabular}{|c|c|c|c|c|c|c|c|c|c|c|c|}
\hline \multirow[t]{2}{*}{$\begin{array}{l}\text { Main } \\
\text { function }\end{array}$} & \multirow{2}{*}{$\begin{array}{r}\begin{array}{r}\text { Condi- } \\
\text { tional }\end{array} \\
\text { si(-qui- } \\
\text { dem })\end{array}$} & \multirow{2}{*}{\begin{tabular}{r|} 
Final \\
ut (and \\
$n e)$
\end{tabular}} & \multirow{2}{*}{$\begin{array}{c}\text { Causal } \\
\text { quia }\end{array}$} & \multirow{2}{*}{$\begin{array}{r}\text { Excep- } \\
\text { tive } \\
\text { nisi }\end{array}$} & \multirow{2}{*}{\begin{tabular}{|r|}
$\begin{array}{r}\text { Com- } \\
\text { parative }\end{array}$ \\
$\begin{array}{r}\text { quasi, } \\
\text { tam- } \\
\text { quam }\end{array}$
\end{tabular}} & \multirow{2}{*}{$\begin{array}{r}\begin{array}{r}\text { Tem- } \\
\text { poral }\end{array} \\
\text { donec, } \\
\text { dum }\end{array}$} & \multirow{2}{*}{$\begin{array}{r}\text { Several } \\
\begin{array}{r}\text { quo- } \\
\text { niam }\end{array}\end{array}$} & \multirow{2}{*}{\begin{tabular}{|r|}
$\begin{array}{r}\text { Conces- } \\
\text { sive }\end{array}$ \\
quam- \\
quam, \\
quam- \\
vis, licet
\end{tabular}} & \multirow{2}{*}{$\begin{array}{r}\begin{array}{r}\text { Sever- } \\
\mathrm{al}^{25}\end{array} \\
\begin{array}{r}\text { cum } \\
(\mathrm{CON})\end{array}\end{array}$} & \multicolumn{2}{|c|}{$\begin{array}{r}\text { PREP of } \\
\text { conformity }\end{array}$} \\
\hline & & & & & & & & & & quoad $^{26}$ & $\begin{array}{r}\text { secun- } \\
\text { dum }\end{array}$ \\
\hline $\begin{array}{l}100 \mathrm{BC}- \\
\mathrm{AD} 200\end{array}$ & 7.33 & 13.26 & 1.00 & 1.56 & 1.55 & 0.69 & 0.79 & 0.84 & 6.31 & 0.075 & 0.14 \\
\hline stdev & 3.00 & 4.27 & 0.83 & 0.63 & 1.39 & 0.58 & 0.63 & 0.47 & 2.20 & 0.101 & 0.20 \\
\hline $\begin{array}{l}100 \mathrm{BC}- \\
A D 200\end{array}$ & 7.26 & 13.88 & 1.08 & 1.63 & 1.29 & 0.64 & 0.67 & 0.81 & 6.94 & 0.098 & 0.16 \\
\hline
\end{tabular}

25 Corpus Corporum cannot distinguish between the two cums. Roelli (2013) determined that an average $74 \%$ of occurrences are of the conjunction; the total number has been multiplied by this value. The accuracy of these numbers is, therefore, worse than that of the others, as the ratio is likely to change depending on the author.

26 Both quoad and quo ad were counted, the latter orthography being rather rarer in our texts. 
Table 10: (continued)

\begin{tabular}{l|r|r|r|r|r|r|r|r|r|r|r}
\hline $\begin{array}{l}200- \\
450\end{array}$ & 6.26 & 12.03 & 5.32 & 1.90 & 1.29 & 0.81 & 1.21 & 0.56 & 5.03 & 0.004 & 1.19 \\
\hline $\begin{array}{l}780- \\
900\end{array}$ & 4.78 & 11.24 & 6.38 & 1.39 & 1.16 & 1.17 & 1.05 & 0.50 & 4.49 & 0.005 & 1.33 \\
\hline $\begin{array}{l}1100- \\
1220\end{array}$ & 4.97 & 10.46 & 5.69 & 1.49 & 1.51 & 1.24 & 0.95 & 0.51 & 5.01 & 0.020 & 1.26 \\
\hline $1500-$ & 2.99 & 11.20 & 2.12 & 0.63 & 0.95 & 0.94 & 0.35 & 0.41 & 4.98 & 0.113 & 0.51 \\
1820 & & & & & & & & & & \\
\hline avg & $\mathbf{5 . 2 3}$ & $\mathbf{1 1 . 7 6}$ & $\mathbf{4 . 1 2}$ & $\mathbf{1 . 4 1}$ & $\mathbf{1 . 2 4}$ & $\mathbf{0 . 9 6}$ & $\mathbf{0 . 8 5}$ & $\mathbf{0 . 5 6}$ & $\mathbf{5 . 2 9}$ & $\mathbf{0 . 0 4 8}$ & $\mathbf{0 . 8 9}$ \\
\hline stdev & 1.65 & 1.31 & 2.36 & 0.48 & 0.21 & 0.25 & 0.34 & 0.15 & 0.95 & 0.053 & 0.52 \\
\hline
\end{tabular}

As expected, many of these numbers are reasonably stable through time (e.g. for quasi, tamquam and cum; slightly falling for ut), but often the stdev is high. Some seem to be especially 'mediaeval': quia, quoniam, and secundum, although the values from the early modern corpus are still a lot higher than the classical ones (except quoniam); quoad behaves in exactly the opposite manner, although it is much rarer. Indeed, quia and quoniam are used much more profusely in the Middle Ages in situations where Antiquity would prefer other constructions, especially the AcI (it was found above that the Middle Ages have conspicuously lower numbers for INF). ${ }^{27}$ The Croatian early modern authors seem to avoid hypotaxis quite generally, possibly because it was felt to be a trait of mediaeval, 'barbarous' Latin. Thus, their numbers for relative sentences and si and nisi are much lower than the mediaeval and the antique values, which are similar to each other.

These numbers obtained from the five large corpora representing five important periods will now serve as benchmark values to compare against the scientific samples.

\section{General scientific corpus}

\$3 The table below (§4) summarises important data about the forty texts chosen for this general, diachronic scientific corpus and provides some initial linguistic information about the texts. ${ }^{28}$ The texts cover rather different topics and may thus

27 See Stotz (1996-2004: IX, §106 = vol. 4, pp. 397-398 on quia; IX, §107 = vol. 4, pp. 398-399 on quoniam). His observation (p. 398) that quoniam remains significantly rarer than quod and quia is confirmed by our numbers.

28 Details about the editions used can be found in the bibliography. All texts are in Corpus Corporum. 
serve to illustrate general trend developments. They were chosen as relatively original treatments of a scientific subject, excluding mere schoolbooks, technical manuals, translations, and texts written in verse. Some scientific texts translated from Greek are included in the study as out-groups: four texts translated under different circumstances and at different times (listed at the end of the table). After this table listing the texts used in the sample, a table with the PoS distribution and another one with the other parameters discussed above for the benchmark samples follow. Average figures of the more homogeneous samples studied in the next chapter are also displayed in the tables: text samples from arithmetic, historiography, and didactic/scientific poetry.

In order to gain an insight into the innovated technical vocabulary, words not occurring in Antiquity were counted in the last 5,000 words (thus avoiding the special language used in prefaces) of each work in this corpus. ${ }^{29}$ In order to find them, an 'Antiquity word-list' was first generated from texts by authors between $100 \mathrm{BC}$ and AD 200 from corpora 4 and 5 in CC (171 texts, yielding some 257,333 words), which was subtracted from the word-lists of later texts in the sample. ${ }^{30}$ The resulting word-lists of post-antique lemmata for each author are printed in appendix 1 at the end of this book. The 31 scientific texts after AD 200 in total contain 917 [3,032] lemmata [occurrences] not extant in the sample for before AD 200. Among these were 294 [1145] ADJ, 20 [37] ADV, 465 [1,544] N, and 138 [306] V. Thus, at least these post-antique words do show a decidedly nominal character. The numbers vary strongly depending on the scientific field. Logical or philosophical texts (Boethius, Anselm, Spinoza) only rarely use new words, unless they belong to university scholasticism (Suárez). Indeed, the author who uses by far the most such words is the twentieth-century Jesuit Boyer. Among the humanist authors, some exhibit lower numbers (Cardano, Vesalius, Descartes, Newton, Kretschmann), but even avowed classicists such as Vesalius cannot help using some late words in a field like medicine. The comparison with the out-groups shows that, interestingly, the Vulgate uses quite precisely the same number of new words as the average of the scientific texts. ${ }^{31}$ Since the numbers depend

29 A similar experiment with only classical authors (Cicero, Caesar, Cornelius Nepos, Pliny the Younger, Quintilian, Ad Herennium, Ps-Caesar, Livy, and Sallust, in total some 54 texts - compare Krebs, who recommends the 'pure' style of the later authors Pliny the Younger and Quintilian) produced too many words, many of which are seemingly completely normal Latin that just happened not to be present in the relatively small sample of 'authoritative' texts. They are not included.

30 Some manual clean-up and lemmatising of words unknown to Corpus Corporum's lemmatiser had to be done.

31 This procedure was also applied to the Vulgate text: 41 lemmata [101 instances] were found, also listed in appendix 1. 
strongly on the scientific field and possibly also on the part of the work from which the 5,000 words were taken, they are not used as a parameter for the plots below. $^{32}$

The list in the appendix shows that Latin scientific language created nouns, adjectives, and verbs in large numbers, but there are very few new uninflected words (aptota). ${ }^{33}$ The only one discussed in this book is the scholastic pseudoarticle ly (see chap. $24 \S 6$ below). It remained marginal and was no longer employed in early modern times. Nevertheless, it will become clear that some aptota are much more common in scientific writing, especially PREP and in some authors ADV. Some cases are conspicuous, for instance secundum. A look at the entries in Schütz yields, besides ly, only aptota that are adverbs derived from adjectives, in -e, -ter, and some less regular ones in -im (capitulatim, coniunctim, copulatim, disiunctim, divisim, subalternatim, summatim). It may be a generalisable finding that technical languages create new vocabulary nearly exclusively among the open (inflectable) word classes, hardly ever among the structure words of a language. The latter would constitute a much deeper intervention in language, on the way toward language engineering.

\$4 There follow the tables with information about the texts used and the figures obtained for them (tables 8-10). The texts of translations from Greek in the second part of the table are included for comparison. The first column indicates the text and edition used for the calculations. Often these are not the latest or best critical editions but those that were available in digital form. ${ }^{34}$ It is not to be expected that the differences would result in greatly differing numerical values. The scientific field with which the text is concerned and the text's style are also briefly described in the table. For deeper information, the reader is referred back to part 2, where the works were discussed. The brief assessment of the type of language used is, of course, rather subjective. It is meant merely as an indication what the Latin these texts use 'feels' like to the reader.

32 If the entire process of finding post-antique lemmata could be automated, average numbers for any sample of 5,000 words could be calculated. But at present, listing these words is still partly manual work.

33 On aptota in Indo-European, see Dunkel (2014).

34 Restrictive copyright laws and editorial policies make it impossible to work with the latest editions in many cases. Of course, the best editions available were used in part 2 above. 


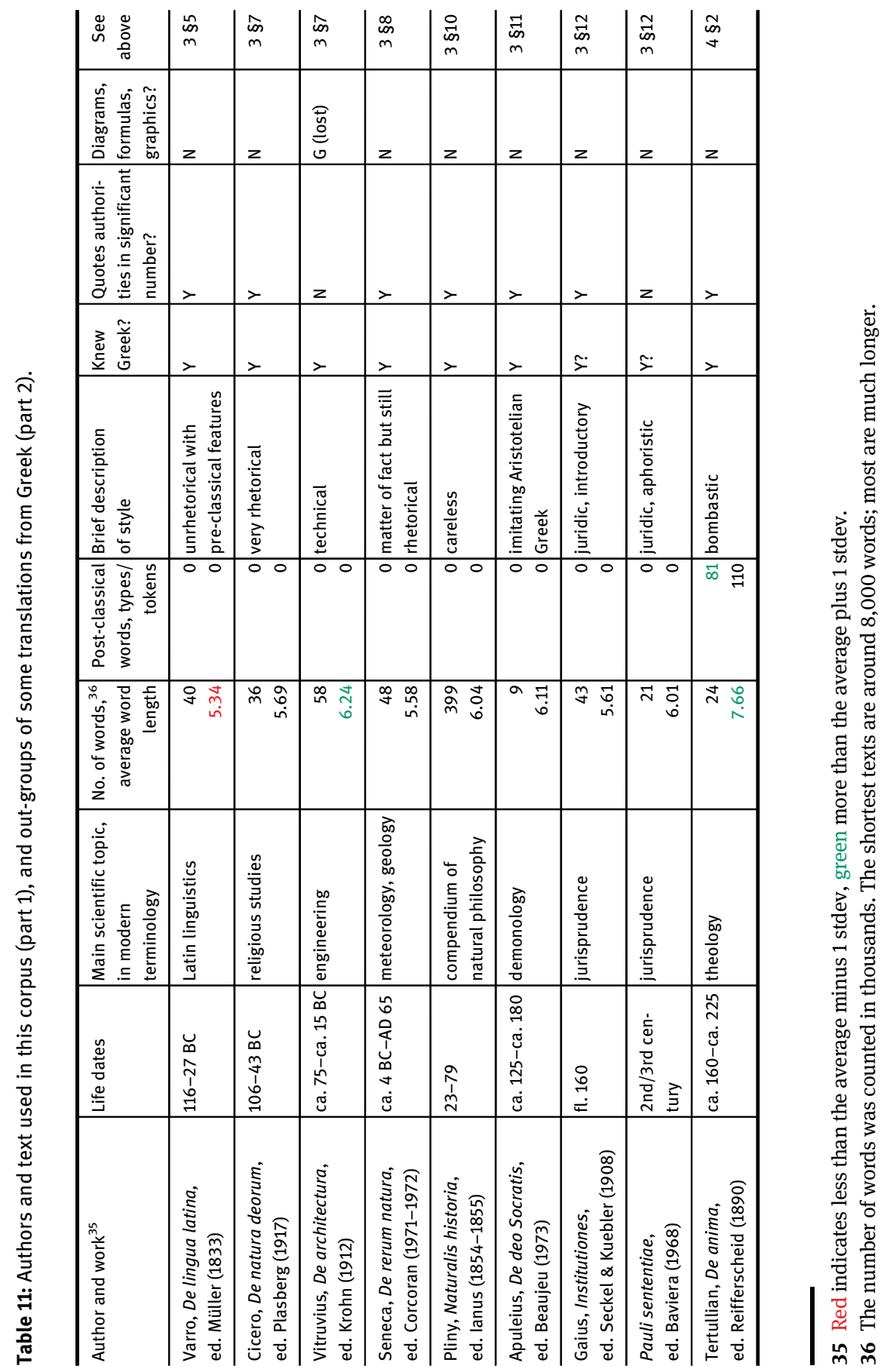




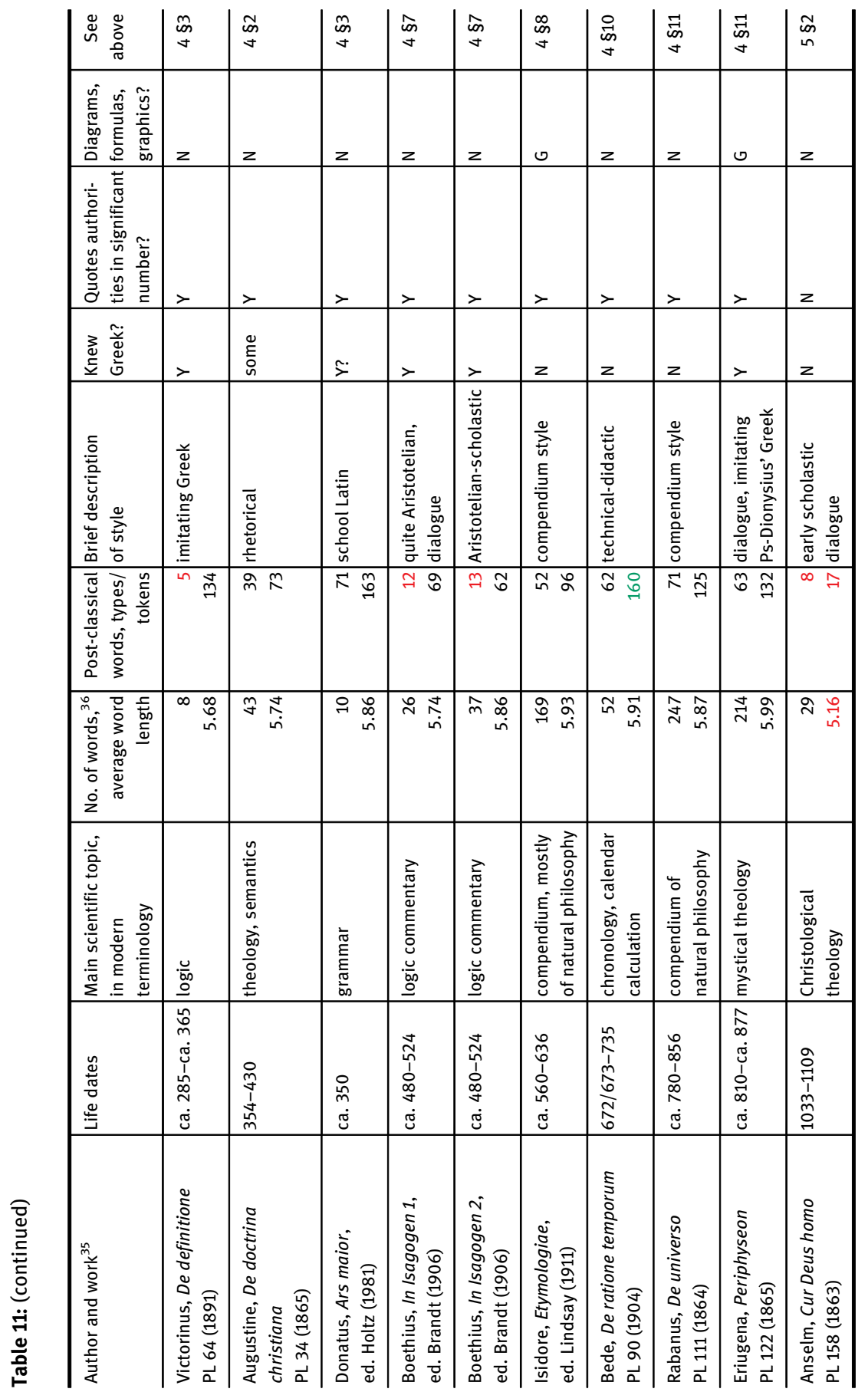




\begin{tabular}{|c|c|c|c|c|c|c|c|c|c|}
\hline 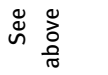 & $\begin{array}{l}\infty \\
\infty \\
\text { in }\end{array}$ & $\begin{array}{l}n \\
\infty \\
\text { in }\end{array}$ & in & in & $\begin{array}{l}\vec{c} \\
\text { in }\end{array}$ & $\begin{array}{c}\infty \\
\infty \\
0\end{array}$ & $\stackrel{\omega_{0}}{\sim}$ & $\underset{\omega}{\omega}$ & $\begin{array}{l}\omega_{0} \\
\omega \\
0\end{array}$ \\
\hline 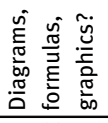 & $z$ & ט & $z$ & $z$ & $z$ & $z$ & 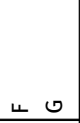 & $z$ & $z$ \\
\hline 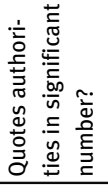 & $z$ & $>$ & $>$ & $>$ & $>$ & $>$ & $z$ & $z$ & $z$ \\
\hline 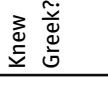 & $z$ & $z$ & $\begin{array}{l}0 \\
\text { Ẽ } \\
\text { in } \\
\end{array}$ & $z$ & $z$ & $\begin{array}{l}0 \\
\text { Eे } \\
\stackrel{n}{n}\end{array}$ & $z$ & $z$ & $z$ \\
\hline 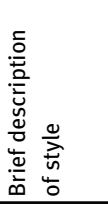 & 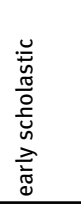 & 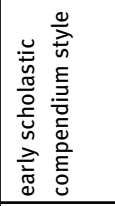 & 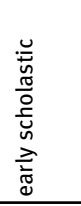 & 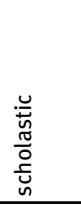 & 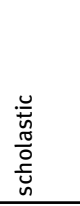 & 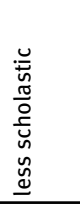 & 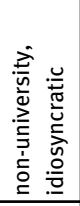 & 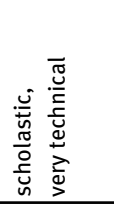 & 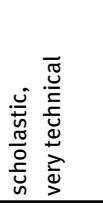 \\
\hline 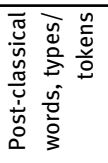 & ని & mे $\infty$ & $\tilde{m} \overrightarrow{0}$ & $\stackrel{m}{\infty}$ & 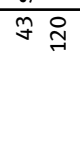 & $\stackrel{n}{n} \stackrel{n}{k}$ & ㅇํㅇㅇㅁㅇ & กิ & $\widehat{m} \vec{\exists}$ \\
\hline 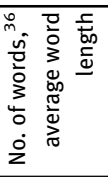 & 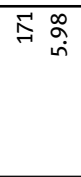 & $\approx \underset{\text { Ni }}{N}$ & ले & 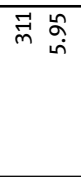 & $\stackrel{\sim}{\infty} \underset{\sim}{\stackrel{N}{\hat{n}}}$ & 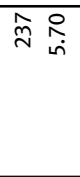 & 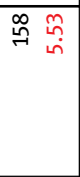 & $\begin{array}{l}\vec{N} \\
\stackrel{a}{a} \\
\text { i }\end{array}$ & à \\
\hline 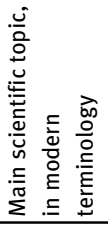 & $\begin{array}{l}\stackrel{u}{\text { ox }} \\
\underline{O}\end{array}$ & 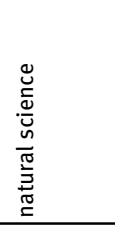 & 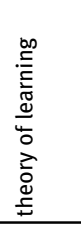 & 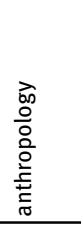 & 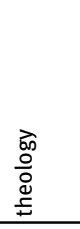 & 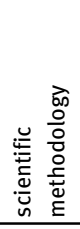 & 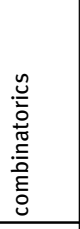 & 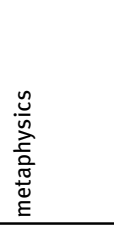 & 品 \\
\hline 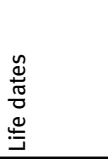 & \begin{tabular}{l}
\multirow{2}{*}{} \\
\multirow{1}{1}{} \\
$o$ \\
$\hat{0}$ \\
\end{tabular} & 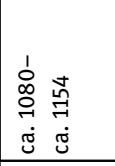 & 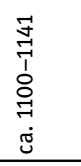 & 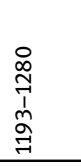 & 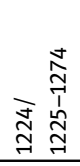 & 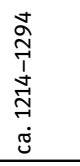 & 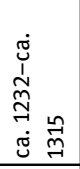 & 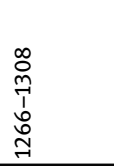 & 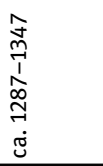 \\
\hline 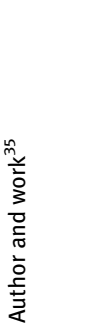 & 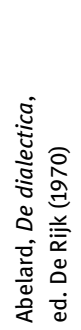 & 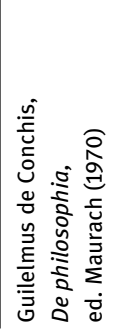 & 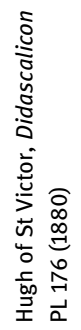 & 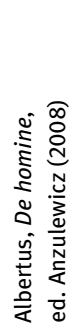 & 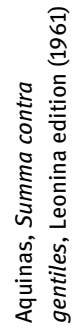 & 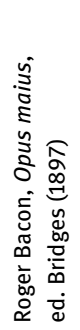 & 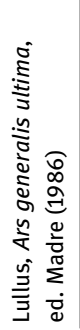 & 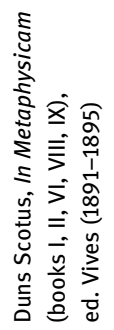 & 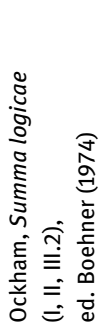 \\
\hline
\end{tabular}




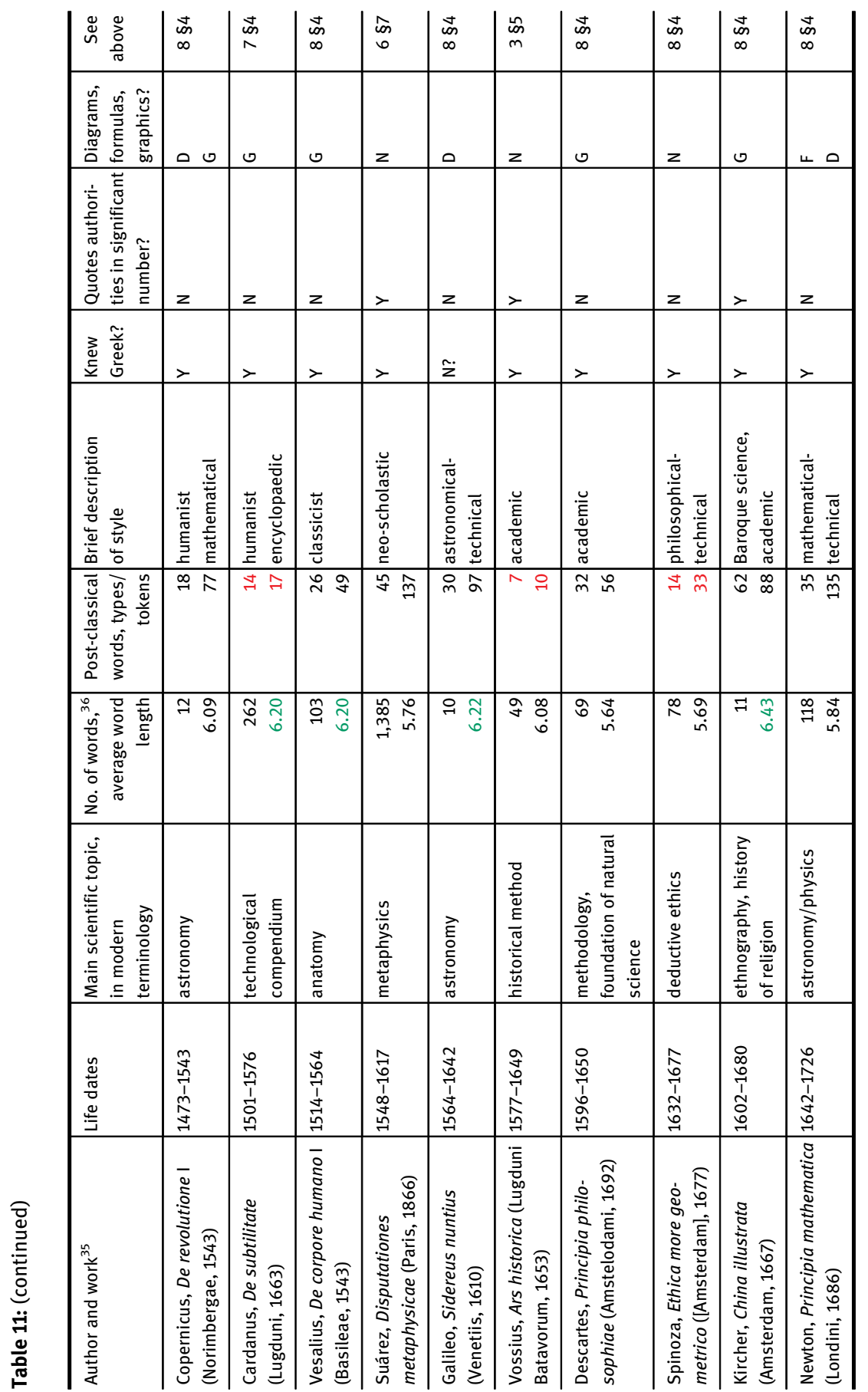




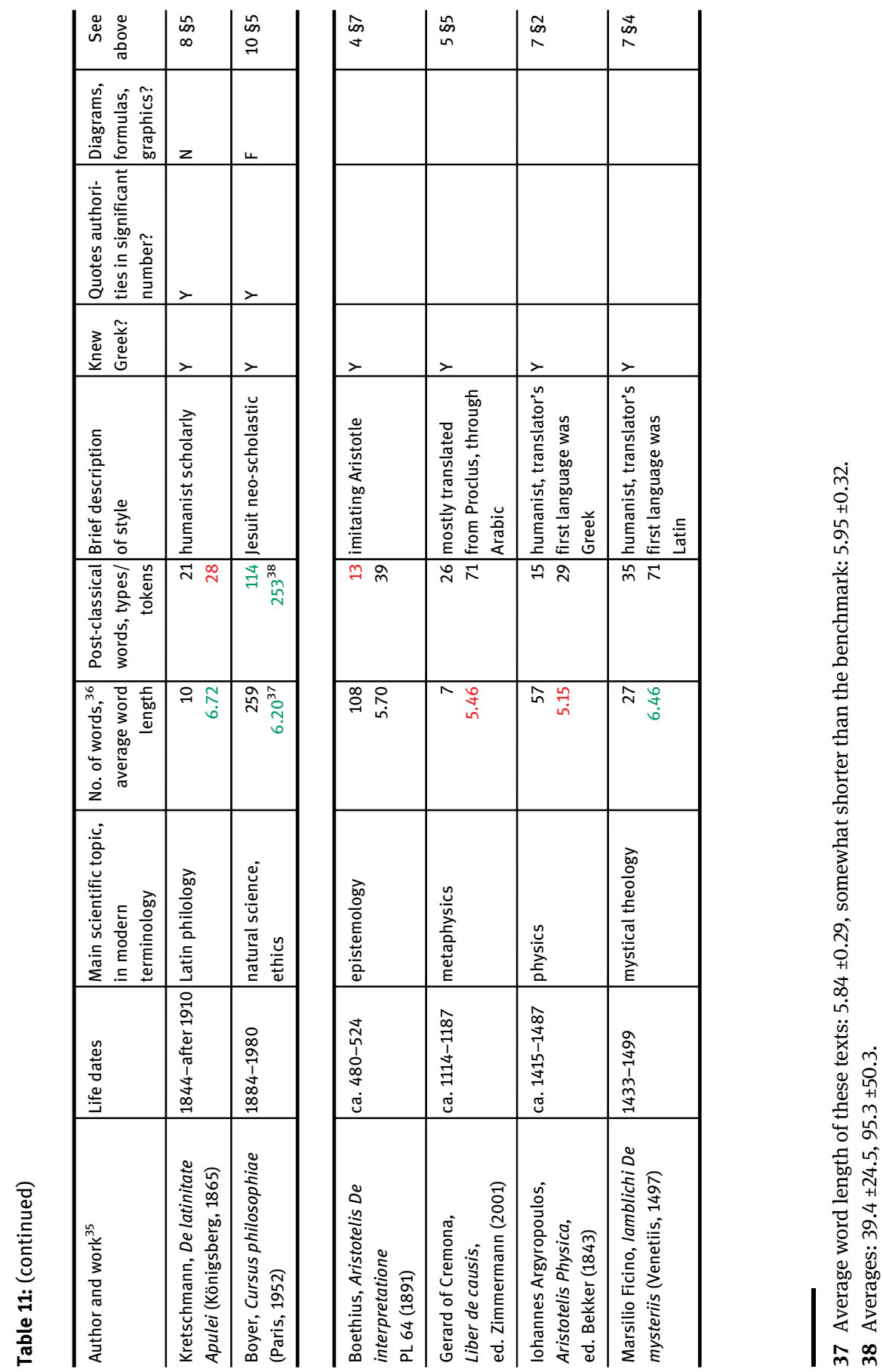


Table 12: PoS values for the scientific texts in comparison to the benchmark general texts, as percentages. The colours green and red are used to show differences from the average of the five prose benchmark texts that exceed $1 \mathrm{stdev} .{ }^{39}$ Values more than 3 times the stdev from the average are underlined. The average and stdev values from the arithmetic corpus (studied in the next chapter) are included for comparison.

\begin{tabular}{|c|c|c|c|c|c|c|c|}
\hline PoS data & ADJ & ADV & CONJ & $\mathbf{N}$ & PREP & PRON & v \\
\hline Varro & 10.85 & 9.08 & 8.97 & 24.58 & 10.56 & 14.46 & 21.50 \\
\hline Cicero & 9.91 & 9.97 & 10.24 & 24.36 & 6.50 & 15.76 & 23.25 \\
\hline Vitruvius & 11.58 & 8.45 & $\underline{8.23}$ & $\underline{30.58}$ & 10.53 & $\underline{8.72}$ & 21.91 \\
\hline Seneca & 11.23 & 10.42 & 9.34 & 22.56 & 7.20 & 13.98 & 25.27 \\
\hline Pliny & 14.40 & 7.14 & $\underline{8.10}$ & 32.75 & 8.79 & $\underline{8.78}$ & 20.03 \\
\hline Apuleius & 13.39 & 7.90 & $\underline{11.55}$ & 26.32 & $\underline{5.50}$ & 12.70 & 22.64 \\
\hline Gaius & 8.49 & 9.73 & 11.02 & 24.79 & 8.16 & 13.96 & 23.85 \\
\hline Pauli sententiae & 9.10 & 6.97 & 9.32 & 29.35 & 8.70 & 10.74 & 25.82 \\
\hline Tertullian & 10.24 & $\underline{11.01}$ & $\underline{13.34}$ & 26.63 & 8.20 & 10.98 & $\underline{19.59}$ \\
\hline Victorinus & 9.55 & 8.70 & 10.08 & 22.23 & 7.70 & 15.38 & $\underline{26.35}$ \\
\hline Augustine & 9.38 & 10.47 & $\underline{11.40}$ & $\underline{21.25}$ & 7.35 & 15.30 & 24.85 \\
\hline Donatus & $\underline{17.73}$ & $\underline{6.52}$ & $\underline{13.66}$ & 24.07 & 8.95 & $\underline{9.60}$ & $\underline{19.48}$ \\
\hline Boethius 1 & 11.04 & 10.22 & $\underline{12.34}$ & 22.29 & 7.52 & 14.58 & 22.01 \\
\hline Boethius 2 & 12.06 & $\underline{11.42}$ & 11.10 & $\underline{21.17}$ & 7.81 & 14.30 & 22.14 \\
\hline Isidore & 10.67 & 7.81 & 9.70 & 29.39 & 8.87 & 10.94 & 22.61 \\
\hline Bede & 16.56 & 7.53 & 7.41 & $\underline{31.16}$ & 8.50 & $\underline{9.66}$ & $\underline{19.19}$ \\
\hline Rabanus & 10.41 & 7.47 & 8.90 & 32.59 & 9.04 & 10.78 & 20.82 \\
\hline Eriugena & 11.67 & 9.98 & 10.90 & 23.73 & 8.85 & 13.71 & 21.16 \\
\hline Anselm & 10.90 & $\underline{12.36}$ & $\underline{12.88}$ & $\underline{16.13}$ & 7.37 & $\underline{18.44}$ & 21.92 \\
\hline Abelard & 10.70 & $\underline{13.07}$ & 10.33 & $\underline{19.52}$ & 9.15 & 14.05 & 23.17 \\
\hline Guil. de Conchis & 11.11 & 10.26 & 10.44 & 23.34 & $\underline{10.55}$ & 11.49 & 22.82 \\
\hline Hugh & 12.36 & 9.57 & 9.44 & 26.49 & 8.13 & 11.06 & 22.95 \\
\hline Albertus & 11.39 & 10.99 & 10.03 & 22.27 & $\underline{11.80}$ & 12.76 & 20.77 \\
\hline Aquinas & 10.38 & $\underline{10.92}$ & 9.07 & 22.17 & 10.52 & 14.58 & 22.36 \\
\hline Roger Bacon & 12.41 & 8.77 & 12.44 & 24.88 & 10.57 & 10.84 & 20.10 \\
\hline
\end{tabular}

39 The samples are treated as a single text. This explains the (small) differences from the numbers in table 6 above. 
Table 12: (continued)

\begin{tabular}{|c|c|c|c|c|c|c|c|}
\hline PoS data & ADJ & ADV & CONJ & $\mathbf{N}$ & PREP & PRON & v \\
\hline Lullus & 11.66 & 8.18 & 10.28 & 22.85 & $\underline{12.82}$ & 12.11 & 22.09 \\
\hline Duns Scotus & $\underline{13.23}$ & $\underline{12.47}$ & 9.60 & $\underline{19.39}$ & $\underline{11.87}$ & 13.28 & 20.16 \\
\hline Ockham & 12.63 & $\underline{11.17}$ & 10.49 & $\underline{18.13}$ & 9.21 & 15.10 & 23.26 \\
\hline Copernicus & $\underline{14.18}$ & 9.40 & $\underline{7.24}$ & 23.68 & $\underline{11.25}$ & 12.92 & 21.33 \\
\hline Cardanus & $\underline{13.00}$ & $\underline{11.59}$ & 10.05 & 26.03 & 8.54 & $\underline{9.65}$ & 21.14 \\
\hline Vesalius & $\underline{16.31}$ & $\underline{11.81}$ & $\underline{7.69}$ & 28.15 & 7.15 & $\underline{10.71}$ & $\underline{18.18}$ \\
\hline Suárez & 11.42 & $\underline{11.46}$ & 12.23 & $\underline{20.53}$ & 10.55 & 12.29 & 21.52 \\
\hline Galileo & $\underline{21.07}$ & $\underline{11.11}$ & $\underline{6.88}$ & 22.24 & 9.79 & $\underline{9.71}$ & $\underline{19.20}$ \\
\hline Vossius & 11.62 & $\underline{10.68}$ & 10.74 & 25.96 & 8.23 & $\underline{10.73}$ & 22.03 \\
\hline Descartes & 11.38 & 11.62 & 9.76 & $\underline{20.65}$ & $\underline{10.39}$ & 15.59 & 20.61 \\
\hline Spinoza & 9.38 & 8.89 & 10.10 & 22.26 & 9.19 & 16.95 & 23.22 \\
\hline Kircher & 14.67 & 8.34 & 4.50 & 27.52 & 9.43 & 13.75 & 21.80 \\
\hline Newton & 13.60 & $\underline{7.02}$ & $\underline{7.09}$ & 27.75 & $\underline{14.14}$ & $\underline{10.52}$ & 19.88 \\
\hline Kretschmann & $\underline{19.01}$ & 10.15 & $\underline{8.46}$ & 25.73 & $\underline{5.72}$ & $\underline{9.95}$ & 20.98 \\
\hline Boyer & $\underline{13.77}$ & 9.09 & 9.25 & 24.15 & 9.97 & 11.06 & 22.72 \\
\hline avg & 12.36 & 9.74 & 9.86 & 24.49 & 9.13 & 12.55 & 21.87 \\
\hline stdev & 2.73 & 1.69 & 1.89 & 3.81 & 1.82 & 2.38 & 1.83 \\
\hline De interpretatione & $\underline{14.25}$ & $\underline{15.30}$ & $\underline{12.42}$ & $\underline{18.19}$ & 6.45 & 16.69 & $\underline{16.70}$ \\
\hline Liber de causis & $\underline{12.72}$ & 9.98 & $\underline{14.07}$ & 22.61 & $\underline{11.05}$ & 16.39 & $\underline{13.18}$ \\
\hline Argyropoulos & 10.75 & $\underline{14.77}$ & $\underline{14.66}$ & $\underline{16.50}$ & 8.29 & $\underline{18.98}$ & $\underline{16.04}$ \\
\hline Ficinus & $\underline{14.85}$ & $\underline{12.05}$ & $\underline{6.87}$ & 25.40 & 9.62 & 12.55 & $\underline{18.66}$ \\
\hline avg & $\underline{13.14}$ & $\underline{13.02}$ & $\underline{12.01}$ & $\underline{20.68}$ & 8.85 & 16.15 & $\underline{16.15}$ \\
\hline stdev & 1.83 & 2.48 & 3.55 & 4.07 & 1.96 & 2.67 & 2.27 \\
\hline
\end{tabular}


Table 12: (continued)

\begin{tabular}{|c|c|c|c|c|c|c|c|}
\hline PoS data & ADJ & ADV & CONJ & $\mathbf{N}$ & PREP & PRON & v \\
\hline $\begin{array}{l}\text { Arithmetic } \\
\text { (chap. 20) }\end{array}$ & $\underline{18.60}$ & 8.86 & 10.82 & $\underline{18.18}$ & $\underline{11.86}$ & 10.98 & 20.69 \\
\hline stdev & 2.83 & 1.69 & 1.59 & 3.32 & 2.17 & 1.39 & 2.53 \\
\hline 100 BC-AD 200 & 10.40 & 9.59 & 9.43 & 23.65 & 7.09 & 15.65 & 24.19 \\
\hline $200-450$ & 8.67 & 9.13 & 10.52 & 24.90 & 7.98 & 14.87 & 23.93 \\
\hline $780-900$ & 10.10 & 8.21 & 10.20 & 27.03 & 8.81 & 13.50 & 22.15 \\
\hline $1100-1220$ & 10.09 & 8.67 & 9.79 & 26.73 & 8.82 & 13.76 & 22.14 \\
\hline $1500-1820$ & 11.05 & 9.17 & 9.73 & 26.11 & 8.82 & 12.87 & 22.25 \\
\hline avg & 10.06 & 8.95 & 9.93 & 25.69 & 8.30 & 14.13 & 22.93 \\
\hline stdev & 0.87 & 0.53 & 0.43 & 1.40 & 0.77 & 1.12 & 1.03 \\
\hline Digesta & 8.45 & 8.03 & 11.08 & 25.52 & 7.79 & 12.30 & 26.84 \\
\hline Vulgate & $\underline{5.81}$ & 8.55 & $\underline{12.26}$ & 24.83 & 9.36 & $\underline{17.73}$ & 21.46 \\
\hline Charter corpus & 11.90 & 7.86 & $\underline{11.61}$ & 26.24 & $\underline{11.10}$ & 12.90 & $\underline{18.38}$ \\
\hline Poetry corpus & $\underline{15.52}$ & $\underline{7.23}$ & $\underline{7.28}$ & $\underline{30.54}$ & $\underline{4.38}$ & $\underline{10.49}$ & 24.57 \\
\hline Vergil/Ovid & 13.90 & $\underline{6.74}$ & $\underline{6.88}$ & $\underline{31.14}$ & $\underline{4.29}$ & 10.42 & 26.62 \\
\hline $\begin{array}{l}\text { Scientific poetry } \\
\text { (chap. 20) }\end{array}$ & $\underline{14.97}$ & 8.51 & $\underline{8.05}$ & 29.67 & $\underline{5.28}$ & $\underline{9.19}$ & 24.33 \\
\hline
\end{tabular}




\begin{tabular}{|c|c|c|c|c|c|c|c|c|c|c|c|c|c|c|c|c|}
\hline 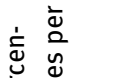 & Entropy & $\begin{array}{l}\infty \\
\infty \\
\infty\end{array}$ & oे & $\stackrel{n}{a}$ & aे & 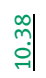 & $\stackrel{\sim}{\sim}$ & $\stackrel{+}{a}$ & $\begin{array}{l}\hat{n} \\
\infty \\
\infty\end{array}$ & $\stackrel{+}{m}$ & बे| & $\begin{array}{l}\text { oे } \\
\text { aे }\end{array}$ & $\begin{array}{l}\mathscr{L} \\
\infty \\
\infty\end{array}$ & ํํ. & 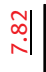 & $\begin{array}{l}\infty \\
\infty \\
\infty \\
\infty\end{array} \mid$ \\
\hline$\frac{\overline{0}}{\frac{0}{2}}$ & ABL ABS & mे| & $\stackrel{\stackrel{v}{m}}{i}$ & 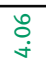 & $\begin{array}{l}\tilde{D} \\
\stackrel{m}{m}\end{array}$ & 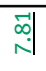 & $\begin{array}{l}\stackrel{0}{0} \\
\dot{m}\end{array}$ & $\stackrel{\substack{\infty \\
\infty}}{i}$ & 羿 & $\begin{array}{l}\text { Lo } \\
\text { in }\end{array}$ & वे| & $\begin{array}{l}\infty \\
\stackrel{\sim}{i} \\
\end{array}$ & 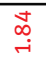 & 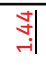 & 웜 & $\begin{array}{c}\infty \\
\substack{\infty \\
\sim}\end{array}$ \\
\hline. & Modifiers & 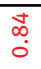 & 离 & $\stackrel{\stackrel{\sim}{\sim}}{\sim}$ & $\stackrel{\infty}{\stackrel{\infty}{+}}$ & $\begin{array}{l}5 \\
0 \\
0 \\
0\end{array}$ & $\begin{array}{l}\overrightarrow{5} \\
\stackrel{+}{+}\end{array}$ & $\begin{array}{l}? \\
i \\
i\end{array}$ & $\begin{array}{l}\begin{array}{l}0 \\
\infty \\
0 \\
0\end{array} \\
\end{array}$ & $\underset{+}{\stackrel{N}{i}}$ & 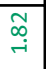 & $\stackrel{\substack{n \\
i}}{ }$ & 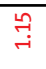 & $\begin{array}{l}\stackrel{\sim}{n} \\
m\end{array}$ & m| & 원 \\
\hline $\begin{array}{l}. \bar{\omega} \\
. \underline{n}\end{array}$ & $\mathrm{~N}$-SUF & 월 & 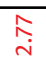 & $\begin{array}{l}\stackrel{n}{\alpha} \\
\stackrel{+}{+}\end{array}$ & ज्ञ & స్ำ & 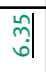 & $\begin{array}{l}\text { mit } \\
\text { in } \\
\text { in }\end{array}$ & 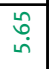 & $\underset{\sim}{\stackrel{\sim}{\checkmark}}$ & \begin{tabular}{l|l|}
$\vec{n}$ \\
$\infty$
\end{tabular} & 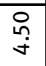 & $\stackrel{\stackrel{n}{n}}{m}$ & $\stackrel{\stackrel{\sim}{n}}{\stackrel{n}{n}}$ & 혐 & 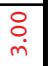 \\
\hline 产 & ADJ-SUF & $\begin{array}{l}\text { ñ } \\
\text { ò }\end{array}$ & $\underset{\sim}{\stackrel{N}{i}}$ & \begin{tabular}{l}
\multirow{2}{*}{} \\
$\stackrel{+}{+}$ \\
\end{tabular} & $\begin{array}{l}\text { के } \\
\stackrel{0}{0}\end{array}$ & शे & 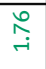 & 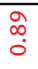 & $\overrightarrow{\vec{m}}$ & $\stackrel{\text { 의 }}{=}$ & $\stackrel{m}{\stackrel{m}{r}}$ & $\underset{\sim}{\stackrel{\sim}{\sim}}$ & mे & సָे & 윙 & $\underset{+}{\stackrel{f}{\rightarrow}}$ \\
\hline$=$ & 3rd PAS & $\begin{array}{l}0 \\
\text { m. } \\
0 \\
0\end{array}$ & $\begin{array}{l}\square \\
\dot{0} \\
\dot{9}\end{array}$ & 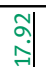 & à & นั้े & $\begin{array}{l}0 \\
\stackrel{0}{0} \\
\dot{9}\end{array}$ & 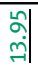 & 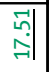 & 궁| & 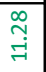 & 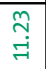 & $\begin{array}{l}\infty \\
\stackrel{\infty}{\infty} \\
\stackrel{-}{-1}\end{array}$ & 帝 & \begin{tabular}{l|l|} 
& \\
0 \\
$\vdots$
\end{tabular} & 형 \\
\hline $\overrightarrow{0}$ & 1st SG & $\begin{array}{l}\tilde{n} \\
\stackrel{n}{n}\end{array}$ & $\begin{array}{l}\infty \\
\infty \\
\dot{J}\end{array}$ & $\begin{array}{l}0 \\
\stackrel{0}{\circ} \\
\stackrel{i}{ }\end{array}$ & $\stackrel{n}{n}$ & $\underset{\rightarrow}{\stackrel{N}{r}}$ & 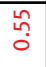 & $\begin{array}{l}\text { Oे } \\
\text { i }\end{array}$ & 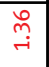 & $\begin{array}{l}\overrightarrow{\hat{m}} \\
\dot{m}\end{array}$ & \begin{tabular}{l}
\multirow{2}{*}{} \\
i \\
\end{tabular} & $\stackrel{N}{N}$ & 包 & $\underset{+}{\stackrel{J}{*}}$ & $\begin{array}{l}N \\
\tilde{O} \\
0\end{array}$ & $\begin{array}{l}\text { oे } \\
\text { o. }\end{array}$ \\
\hline Dे & PRON:POSS & 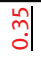 & 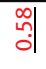 & 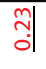 & \begin{tabular}{|l|}
$\overrightarrow{0}$ \\
0 \\
0
\end{tabular} & ले. & ज़ี & $\begin{array}{l}\mid \\
\\
\infty \\
0 \\
\vdots\end{array}$ & |: & ㅊ: & ज़| & $\begin{array}{l} \\
0 \\
\vdots \\
\vdots\end{array}$ & ma. & సֶ. & ㄱํㅇ & गे. \\
\hline 凹్ల & CONJ:S & 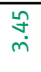 & 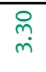 & $\begin{array}{l}\text { o } \\
\text { i }\end{array}$ & 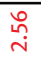 & 굑 & $\begin{array}{l}+ \\
\stackrel{+}{+}\end{array}$ & 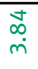 & $\stackrel{9}{\stackrel{9}{m}}$ & 윔 & $\begin{array}{l}\vec{b} \\
\dot{m}\end{array}$ & $\begin{array}{l}\vec{t} \\
\dot{m} \\
\dot{m}\end{array}$ & $\begin{array}{l}\text { of } \\
\text { जै| }\end{array}$ & $\begin{array}{l}\overrightarrow{\hat{n}} \\
\dot{m}\end{array}$ & $\begin{array}{l}\hat{\infty} \\
\dot{m}\end{array}$ & $\underset{i}{\mathbb{N}}$ \\
\hline$\frac{\varrho}{\frac{0}{E}}$ & REL & 总 & $\begin{array}{l}\overrightarrow{0} \\
\dot{\sigma} \\
\dot{J}\end{array}$ & $\begin{array}{l}\vec{m} \\
\dot{m}\end{array}$ & $\underset{m}{N}$ & 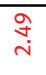 & $\underset{m}{N}$ & $\begin{array}{l}\stackrel{m}{o} \\
\dot{r}\end{array}$ & $\begin{array}{l}\text { \& } \\
\dot{m}\end{array}$ & $\begin{array}{l}\overrightarrow{9} \\
\stackrel{m}{m}\end{array}$ & \begin{tabular}{l}
\multirow{2}{*}{} \\
\multirow{4}{*}{} \\
\end{tabular} & $\begin{array}{l}\hat{N} \\
\dot{\sigma}\end{array}$ & 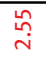 & 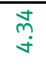 & 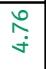 & $\underset{\substack{\text { f } \\
\text { f }}}{ }$ \\
\hline$\stackrel{\Xi}{\sigma}$ & ESSE & $\stackrel{m}{\stackrel{m}{m}}$ & 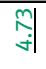 & 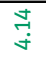 & $\begin{array}{l}\stackrel{p}{m} \\
\stackrel{\sim}{*}\end{array}$ & 곅 & 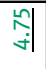 & 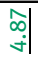 & 坴 & 年 & 위 & $\begin{array}{l}\stackrel{\sim}{\sim} \\
\dot{\forall}\end{array}$ & 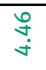 & ํํํ & 앵 & 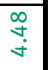 \\
\hline$\frac{d}{\frac{D}{\sigma}} \frac{0}{\frac{0}{0}}$ & ABL/DAT & $\begin{array}{l}0 \\
\stackrel{0}{0} \\
\dot{m}\end{array}$ & $\begin{array}{l}m \\
\hat{i} \\
\dot{m}\end{array}$ & $\begin{array}{l}\infty \\
0 \\
0 \\
\dot{m}\end{array}$ & $\begin{array}{l}m \\
\infty \\
\dot{\sim} \\
\dot{n}\end{array}$ & $\begin{array}{l}\text { वे } \\
\text { के }\end{array}$ & ज्ञ & $\begin{array}{l}\hat{m} \\
\hat{m} \\
m\end{array}$ & $\begin{array}{l}\alpha \\
\hat{m} \\
\vec{m}\end{array}$ & ঐે & 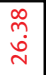 & $\begin{array}{l}\mathbf{1} \\
\stackrel{1}{0} \\
\dot{m}\end{array}$ & 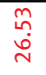 & 离 & \begin{tabular}{l}
\multirow{2}{*}{} \\
ते
\end{tabular} & 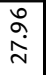 \\
\hline 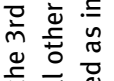 & $\overline{\mathrm{ACC}}$ & $\begin{array}{l}\widehat{0} \\
\dot{\infty} \\
\stackrel{N}{0}\end{array}$ & $\underset{\substack{n \\
\infty}}{\stackrel{\infty}{N}}$ & $\begin{array}{l}\hat{0} \\
\infty \\
\sim \\
N\end{array}$ & |⿱宀寸: & 氮 & $\begin{array}{l}\stackrel{n}{n} \\
\stackrel{m}{m}\end{array}$ & $\begin{array}{l}\text { 命 } \\
\text { in }\end{array}$ & 胥 & $\begin{array}{l}\vec{f} \\
\dot{0} \\
\stackrel{0}{0}\end{array}$ & 离| & $\stackrel{\infty}{\stackrel{\infty}{\sim}}$ & 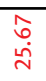 & 年: & 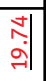 & व్d \\
\hline 8 & $\overline{\text { GEN }}$ & 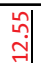 & 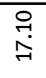 & 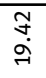 & 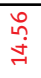 & 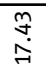 & $\begin{array}{l}\infty \\
\stackrel{\infty}{+} \\
\vec{i}\end{array}$ & 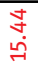 & $\begin{array}{l}\text { 守 } \\
\stackrel{A}{+}\end{array}$ & $\begin{array}{l}\hat{\alpha} \\
\vec{\lambda}\end{array}$ & \begin{tabular}{l}
$\stackrel{n}{n}$ \\
$\ddot{g}$ \\
\cline { 1 - 1 }
\end{tabular} & $\begin{array}{l}0 \\
0 \\
\infty \\
\end{array}$ & 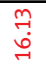 & 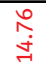 & $\begin{array}{l}\text { जे } \\
\text { जे }\end{array}$ & 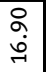 \\
\hline 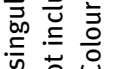 & NOM & $\begin{array}{l}\text { चै| } \\
\text { ○े }\end{array}$ & वे & กิ| & $\begin{array}{l}\stackrel{0}{*} \\
\stackrel{\leftrightarrow}{n}\end{array}$ & 웜 & $\begin{array}{l}\text { 寸े } \\
\text { aे }\end{array}$ & $\begin{array}{l}\stackrel{\infty}{m} \\
\stackrel{\sim}{\sim}\end{array}$ & $\begin{array}{l}\stackrel{0}{+} \\
\text { N }\end{array}$ & $\begin{array}{l}\tilde{m} \\
\dot{\sim}\end{array}$ & 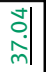 & $\begin{array}{l}\stackrel{\circ}{+} \\
\stackrel{+}{\sim}\end{array}$ & $\begin{array}{l}{ }^{\circ} \\
\text { m. } \\
\text { m. }\end{array}$ & 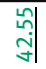 & \begin{tabular}{l|}
$\vec{b}$ \\
$\hat{m}$
\end{tabular} & $\begin{array}{l}\text { స̂. } \\
\text { ֻे }\end{array}$ \\
\hline$\dot{0}$ & PTC & $\begin{array}{l}0 \\
\stackrel{0}{0} \\
\dot{\sigma}\end{array}$ & $\begin{array}{l}m \\
\infty \\
\omega \\
n\end{array}$ & 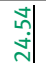 & $\begin{array}{l}a \\
\infty \\
\text { bे }\end{array}$ & $\begin{array}{l}\hat{0} \\
\text { N }\end{array}$ & ๙ু & $\begin{array}{l}\text { बे } \\
\text { जे }\end{array}$ & $\begin{array}{l}\text { ஸे } \\
\stackrel{\sim}{N}\end{array}$ & $\begin{array}{l}\text { స్ } \\
\stackrel{\infty}{\sim}\end{array}$ & $\begin{array}{l}\overrightarrow{7} \\
\vec{n}\end{array}$ & $\begin{array}{l}\underset{\infty}{\infty} \\
\stackrel{?}{0} \\
\sim\end{array}$ & $\begin{array}{l}\overrightarrow{\tilde{n}} \\
\infty \\
\sim \\
-1\end{array}$ & \begin{tabular}{l}
$\infty$ \\
\multirow{2}{0}{} \\
0
\end{tabular} & 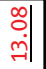 & $\begin{array}{l}\hat{b} \\
\ddot{\lambda}\end{array}$ \\
\hline $\overrightarrow{0}$ & INF & $\begin{array}{l}\overrightarrow{\vec{b}} \\
\stackrel{\sim}{\mathrm{j}}\end{array}$ & $\begin{array}{l}\tilde{N} \\
\stackrel{N}{A}\end{array}$ & $\begin{array}{l}\qquad 0 \\
0 \\
a\end{array}$ & 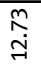 & $\begin{array}{l}\hat{N} \\
\vec{g}\end{array}$ & $\begin{array}{l}\text { aे } \\
\text { 이 }\end{array}$ & 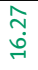 & $\underset{\stackrel{\infty}{\sim}}{\stackrel{\sim}{\sim}}$ & 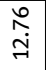 & $\begin{array}{l}\vec{\infty} \\
\vec{\forall}\end{array}$ & $\begin{array}{l}\stackrel{\infty}{\leftrightarrows} \\
\underset{\sim}{\sharp}\end{array}$ & $\begin{array}{l}\infty \\
0 \\
0 \\
0\end{array}$ & 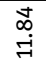 & 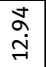 & गे. \\
\hline & SUB & $\begin{array}{l}\stackrel{N}{N} \\
\dot{M}\end{array}$ & $\begin{array}{l}\tilde{L} \\
\infty \\
\infty \\
\sim\end{array}$ & $\begin{array}{l}\hat{\alpha} \\
\hat{n}\end{array}$ & $\begin{array}{l}\hat{L} \\
\ddot{n}\end{array}$ & $\begin{array}{l}\hat{\kappa} \\
\hat{a}\end{array}$ & $\begin{array}{l}\stackrel{a}{+} \\
\dot{\sim}\end{array}$ & $\begin{array}{l}\vec{n} \\
\infty_{0}^{0} \\
\rightarrow\end{array}$ & $\begin{array}{l}\dot{\infty} \\
\ddot{q} \\
\dot{q}\end{array}$ & 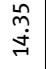 & $\begin{array}{l}\stackrel{m}{m} \\
\stackrel{f}{f}\end{array}$ & \begin{tabular}{l}
$\checkmark$ \\
\multirow{a}{*}{} \\
$\stackrel{n}{*}$
\end{tabular} & 常 & $\begin{array}{l}\infty \\
\text { مे } \\
\text { ஸे }\end{array}$ & $\begin{array}{l}\stackrel{\infty}{m} \\
\stackrel{n}{n}\end{array}$ & $\begin{array}{l}0 \\
\text { in } \\
\text { on }\end{array}$ \\
\hline & IND & $\begin{array}{l}\hat{n} \\
\text { nn }\end{array}$ & \begin{tabular}{l}
$\stackrel{\tilde{n} n}{+}$ \\
\multirow{j}{y}{}
\end{tabular} & 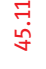 & $\begin{array}{l}n \\
0 \\
\dot{0} \\
\end{array}$ & $\underset{⿱}{\stackrel{f}{+}}$ & 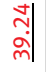 & \begin{tabular}{l}
$\hat{0}$ \\
\multirow{f}{*}{}
\end{tabular} & $\begin{array}{l}\vec{m} \\
\dot{b} \\
\dot{y}\end{array}$ & $\begin{array}{l}\text { ?े } \\
\text { on }\end{array}$ & $\begin{array}{l}\tilde{D} \\
\stackrel{\sim}{n} \\
\dot{n}\end{array}$ & $\begin{array}{l}\stackrel{2}{\sim} \\
\infty \\
\infty \\
+\end{array}$ & 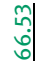 & मे. & $\begin{array}{l}\vec{\infty} \\
\stackrel{0}{n} \\
\stackrel{n}{n}\end{array}$ & 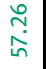 \\
\hline 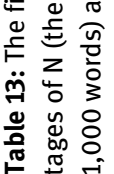 & $\underset{e}{\mathbb{x}}$ & 인 & & 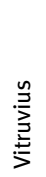 & 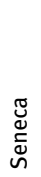 & $\stackrel{\overrightarrow{\underline{\Xi}}}{a}$ & $\frac{\frac{n}{\omega}}{\frac{a}{2}}$ & 胥 & 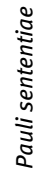 & 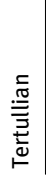 & 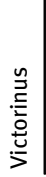 & 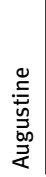 & 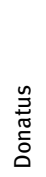 & 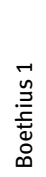 & 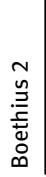 & $\begin{array}{l}\frac{0}{0} \\
\frac{0}{0} \underline{\underline{n}}\end{array}$ \\
\hline
\end{tabular}




\begin{tabular}{|c|c|c|c|c|c|c|c|c|c|c|c|c|c|c|c|c|}
\hline Entropy & 売 & | & $\begin{array}{l}\tilde{n} \\
\infty\end{array}$ & 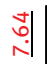 & त्र| & $\begin{array}{l}0 \\
0 \\
\infty\end{array} \mid$ & $\begin{array}{l}\text { ָे } \\
a\end{array}$ & 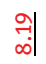 & $\begin{array}{c}\stackrel{1}{0} \\
\infty\end{array} \mid$ & $\stackrel{\infty}{a}$ & 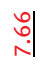 & 辛 & $\stackrel{\substack{n \\
N}}{\mid}$ & 苟 & $\begin{array}{l}\stackrel{N}{a} \\
\sigma\end{array}$ & 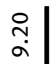 \\
\hline ABL ABS & 㺃 & $\begin{array}{l}\stackrel{\text { 年 }}{\mathrm{N}} \\
\end{array}$ & $\underset{\underset{N}{N}}{\stackrel{N}{N}}$ & $\begin{array}{c}\tilde{c} \\
0 \\
\vdots \\
0\end{array}$ & $\begin{array}{l}0 \\
\stackrel{\infty}{\sim} \\
\rightarrow \mid\end{array}$ & 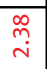 & 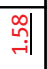 & $\begin{array}{l}\text { ñ. } \\
\text { d. }\end{array}$ & \begin{tabular}{l|l|}
\multirow{7}{*}{$\mid$} \\
|
\end{tabular} & $\stackrel{\infty}{\stackrel{\infty}{\rightarrow}}$ & ले| & 엥 & $\begin{array}{c}\stackrel{m}{N} \\
-1\end{array}$ & $\stackrel{\stackrel{n}{m}}{m}$ & $\begin{array}{l}\dot{m} \\
\dot{m}\end{array}$ & $\begin{array}{l}m \\
\dot{m} \\
\dot{m}\end{array}$ \\
\hline Modifiers & 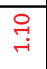 & 각 & $\begin{array}{l}\stackrel{\stackrel{N}{N}}{\sim} \\
\end{array}$ & $\begin{array}{l}\text { I্ } \\
\text { il }\end{array}$ & $\begin{array}{l}\stackrel{\text { ㄱ}}{\sim} \\
\text {. }\end{array}$ & ঐ̊ & $\stackrel{\text { 옥 }}{\rightarrow}$ & స̂̀ & 윔 & $\begin{array}{l}\hat{m} \\
\stackrel{\leftrightarrow}{r}\end{array}$ & $\begin{array}{l}\text { Ju } \\
\text { : } \\
0\end{array}$ & $\underset{\sim}{\stackrel{\leftrightarrow}{\sim}}$ & $\begin{array}{l}\stackrel{0}{\circ} \\
\stackrel{\rightarrow}{0}\end{array}$ & 궉 & 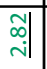 & $\underset{\sim}{\stackrel{\sim}{\rightarrow}}$ \\
\hline $\mathrm{N}$-SUF & $\begin{array}{l}\infty \\
\stackrel{0}{\sim} \\
\dot{N}\end{array}$ & $\underset{\sim}{\vec{\forall}}$ & 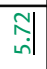 & $\begin{array}{l}\infty \\
\stackrel{\infty}{+} \\
\dot{f}\end{array}$ & 곗 & $\stackrel{\text { 움 }}{\stackrel{4}{+}}$ & $\begin{array}{l} \\
\text { in } \\
\text { in }\end{array}$ & 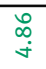 & $\begin{array}{l}\tilde{N} \\
\dot{\omega}\end{array}$ & 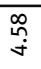 & 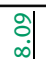 & $\begin{array}{l} \\
0 \\
0 \\
0\end{array} \mid$ & $\begin{array}{l}\vec{m} \\
\dot{\omega n}\end{array}$ & $\begin{array}{l}\stackrel{N}{n} \\
\stackrel{m}{n}\end{array}$ & 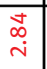 & $\begin{array}{l} \\
\stackrel{2}{\rightarrow} \\
\rightarrow \mid\end{array}$ \\
\hline ADJ-SUF & 엥 & $\begin{array}{l}\text { 우 } \\
\text { G } \\
\text { - }\end{array}$ & जै| & $\begin{array}{l}\text { ڤ̊ } \\
\vdots \\
0\end{array}$ & \begin{tabular}{l|l} 
\\
\end{tabular} & 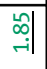 & ํํำ & 号| & \begin{tabular}{ll} 
& \multicolumn{2}{|c|}{$\mid$} \\
$\vdots$ &
\end{tabular} & $\stackrel{\mathrm{m}}{\mathrm{N}}$ & $\overrightarrow{\mathrm{j}} \mid$ & 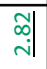 & $\begin{array}{l}\overrightarrow{0} \\
\dot{m} \mid\end{array}$ & 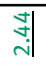 & $\begin{array}{l}\stackrel{\circ}{\rightarrow} \\
\stackrel{\leftrightarrow}{\rightarrow}\end{array}$ & $\underset{\sim}{\stackrel{ }{\sim}}$ \\
\hline 3rd PAS & $\begin{array}{l}\infty \\
0 \\
0 \\
0\end{array}$ & 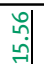 & \begin{tabular}{l} 
Na \\
\multirow{7}{*}{}
\end{tabular} & $\begin{array}{l}\hat{f} \\
\sigma\end{array}$ & 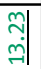 & $\begin{array}{l}\overrightarrow{1} \\
\infty \\
\rightarrow \\
ت\end{array}$ & $\begin{array}{l}\vec{v} \\
m \\
\stackrel{a}{0}\end{array}$ & \begin{tabular}{l} 
\\
0 \\
0 \\
$\vdots$ \\
\hdashline
\end{tabular} & $\begin{array}{l}\text { mo| } \\
\stackrel{n}{\mid} \mid\end{array}$ & $\begin{array}{l}{ }^{\infty} \\
\stackrel{-}{\rightarrow}\end{array}$ & 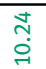 & $\begin{array}{l}0 \\
0 \\
0 \\
0\end{array}$ & $\begin{array}{l}0 \\
0 \\
\dot{m}\end{array}$ & $\begin{array}{l}\text { वे| } \\
\text { वे| }\end{array}$ & 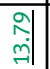 & $\begin{array}{ll} & \\
0 & \\
0\end{array}$ \\
\hline 1st SG & $\begin{array}{l}\stackrel{2}{\sim} \\
\rightarrow\end{array}$ & $\underset{\rightarrow}{\stackrel{m}{\sim}}$ & 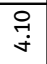 & 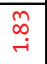 & बू. & $\begin{array}{l}\text { ָे } \\
\text { Oे }\end{array}$ & $\underset{\mathcal{N}}{\mathcal{N}}$ & $\begin{array}{l}0 \\
\stackrel{0}{0} \\
0\end{array}$ & $\begin{array}{l}\hat{n} \\
\tilde{0} \\
0\end{array}$ & $\underset{\sim}{\stackrel{N}{r}}$ & ָ̦ & $\underset{\sim}{\stackrel{N}{r}}$ & $\underset{\rightarrow}{\mathcal{F}}$ & $\begin{array}{l}\overrightarrow{+} \\
\overrightarrow{+}\end{array}$ & 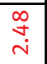 & $\begin{array}{l}\checkmark \\
\dot{v} \\
\dot{m}\end{array}$ \\
\hline PRON:POSS & $\begin{array}{l}\text { n̂n } \\
\text { d. }\end{array}$ & 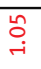 & $\begin{array}{l}\hat{O} \\
\stackrel{+}{1}\end{array}$ & ली. & m. & กี้ & $\begin{array}{l}\hat{0} \\
0 \\
0\end{array}$ & 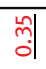 & \begin{tabular}{ll|} 
& \multicolumn{1}{|c}{$\mid$} \\
0 \\
0
\end{tabular} \mid & $\begin{array}{l}\text { mof } \\
0\end{array}$ & $\stackrel{\stackrel{\sim}{m}}{\rightarrow}$ & స્| & 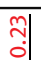 & $\begin{array}{c}\infty \\
\text { ô. } \\
0\end{array}$ & 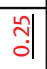 & $\begin{array}{l} \\
\vdots \\
\vdots\end{array}$ \\
\hline CONJ:S & $\begin{array}{l}\vec{t} \\
\stackrel{\rightarrow}{+}\end{array}$ & 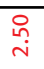 & ঐे & $\begin{array}{l}\text { f) } \\
\stackrel{y}{*} \mid\end{array}$ & $\stackrel{\hat{N}}{m}$ & $\begin{array}{l}\infty \\
0 \\
\stackrel{m}{0}\end{array}$ & $\begin{array}{l}\circ \\
\dot{m}\end{array}$ & $\begin{array}{l}\vec{J} \\
\stackrel{i}{i}\end{array}$ & 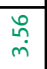 & $\begin{array}{l}\stackrel{0}{\infty} \\
\stackrel{\sim}{N}\end{array}$ & 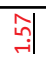 & $\begin{array}{l}\stackrel{P}{1} \\
m\end{array}$ & $\begin{array}{l}\stackrel{8}{ } \\
\dot{m}\end{array}$ & 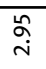 & 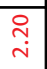 & 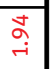 \\
\hline REL & $\stackrel{\substack{m \\
m}}{.}$ & $\begin{array}{l}\stackrel{P}{\infty} \\
\dot{m}\end{array}$ & $\begin{array}{l}\text { Oे } \\
\text { min }\end{array}$ & 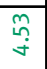 & 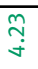 & $\begin{array}{l}\hat{f} \\
\dot{m}\end{array}$ & $\begin{array}{l}\vec{G} \\
\dot{\square}\end{array}$ & $\begin{array}{l}\text { ò } \\
\text { in }\end{array}$ & $\begin{array}{l}\vec{H} \\
\dot{n}\end{array}$ & $\begin{array}{l}\hat{m} \\
\stackrel{m}{m}\end{array}$ & \begin{tabular}{l} 
১े \\
\multirow{্}{*}{}
\end{tabular} & $\begin{array}{l}\stackrel{t}{\infty} \\
\dot{m}\end{array}$ & $\begin{array}{l}\text { సे } \\
\text { ( }\end{array}$ & $\begin{array}{l}\hat{0} \\
\dot{\mathrm{n}}\end{array}$ & $\stackrel{\substack{\infty \\
m}}{ }$ & 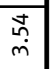 \\
\hline ESSE & ה. & $\hat{\hat{m}}$ & 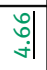 & 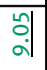 & ఫे| & $\begin{array}{l}\text { Nin } \\
\text { | }\end{array}$ & $\begin{array}{l}2 \\
0 \\
+\end{array}$ & 忒 & $\begin{array}{l}\text { ?ำ } \\
0\end{array}$ & $\begin{array}{l}\vec{m} \\
\dot{\sim}\end{array}$ & 劣 & $\vec{m} \mid$ & 이 & 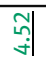 & $\begin{array}{l}\stackrel{ }{\rightarrow} \\
\stackrel{+}{*}\end{array}$ & \begin{tabular}{l|} 
걱 \\
|
\end{tabular} \\
\hline$\overline{A B L / D A T}$ & 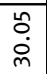 & $\begin{array}{l}\text { Ņ} \\
\text { N }\end{array}$ & $\begin{array}{l}\infty \\
\infty \\
\stackrel{\sim}{N}\end{array}$ & $\begin{array}{l}\underset{N}{N} \\
\stackrel{N}{N}\end{array}$ & 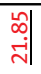 & $\begin{array}{l}\overrightarrow{1} \\
\stackrel{0}{0} \\
m\end{array}$ & $\begin{array}{l}\text { 守 } \\
\stackrel{\text { id }}{N}\end{array}$ & $\begin{array}{l}\stackrel{2}{2} \\
\dot{0}\end{array}$ & 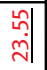 & $\begin{array}{l}\stackrel{\rho}{2} \\
\dot{\omega} \\
N\end{array}$ & $\begin{array}{l}\stackrel{0}{\sim} \\
\stackrel{\sim}{n}\end{array}$ & $\begin{array}{l}0 \\
\infty \\
\infty \\
0 \\
N\end{array}$ & $\begin{array}{l}\stackrel{M}{N} \\
\stackrel{\sim}{N}\end{array}$ & \begin{tabular}{l}
$\tilde{N}$ \\
\multirow{\sim}{*}{}
\end{tabular} & $\begin{array}{l}+ \\
0 \\
\stackrel{j}{m} \\
\tilde{m}\end{array}$ & 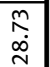 \\
\hline $\mathrm{ACC}$ & $\begin{array}{l}\text { in } \\
\text { Lे } \\
\text { N. }\end{array}$ & 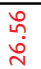 & 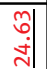 & $\begin{array}{l}\stackrel{2}{2} \\
\stackrel{2}{\mathrm{~N}}\end{array}$ & 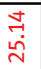 & $\begin{array}{l}\overrightarrow{7} \\
\vec{j}\end{array}$ & $\begin{array}{l}\text { Na } \\
\text { ¿ें } \\
\text { N. }\end{array}$ & \begin{tabular}{l} 
त̃ \\
\multirow{N}{*}{}
\end{tabular} & $\begin{array}{l}\text { ñ } \\
\stackrel{n}{n} \\
\end{array}$ & $\begin{array}{l}\stackrel{\tilde{n}}{\hat{n}} \\
\stackrel{\sim}{N}\end{array}$ & \begin{tabular}{l} 
Na \\
\multirow{\sim}{*}{}
\end{tabular} & \begin{tabular}{l|} 
\\
$\vec{n}$ \\
$\vec{\lambda}$
\end{tabular} & $\begin{array}{l}2 \\
0 \\
0 \\
\oplus \mid\end{array}$ & $\begin{array}{l}\text { नै। } \\
\text { ले| }\end{array}$ & $\begin{array}{l}\text { वे } \\
\text { जो| }\end{array}$ & \begin{tabular}{l|} 
? \\
$\stackrel{+}{*}$
\end{tabular} \\
\hline GEN & $\begin{array}{l}\stackrel{m}{\mathrm{~N}} \\
\stackrel{\sim}{\mathrm{N}}\end{array}$ & \begin{tabular}{l}
\multirow{N}{*}{} \\
$\dot{\sim}$
\end{tabular} & $\begin{array}{l}0 \\
\stackrel{\sim}{\mathbf{N}} \\
\end{array}$ & $\begin{array}{l} \\
\text { oे } \\
\text { gे|l }\end{array}$ & $\begin{array}{l}\stackrel{2}{\wedge} \\
\stackrel{\sim}{\sim}\end{array}$ & 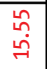 & \begin{tabular}{l|} 
\\
$\infty$ \\
$\infty$ \\
$\sim$ \\
$\sim$
\end{tabular} & $\begin{array}{l}\square \\
0 \\
0 \\
\dot{\sigma}\end{array}$ & $\begin{array}{l}0 \\
m \\
\infty \\
\sim\end{array}$ & 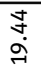 & \begin{tabular}{l}
$\dot{0}$ \\
\multirow{y}{*}{} \\
$\dot{y}$
\end{tabular} & $\begin{array}{l}\stackrel{\sim}{\sim} \\
\stackrel{\sim}{\sim}\end{array}$ & $\begin{array}{l}\infty \\
\stackrel{\infty}{ } \\
\rightarrow \\
\Rightarrow\end{array}$ & $\begin{array}{l}{ }_{\stackrel{\infty}{ }} \\
\stackrel{\sim}{N}\end{array}$ & 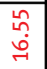 & 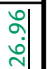 \\
\hline NOM & $\begin{array}{l}\hat{0} \\
\dot{\lambda}\end{array}$ & $\begin{array}{l}\text { fे } \\
\text { Dे }\end{array}$ & $\begin{array}{l}\text { 岁 } \\
\text { 嵓 }\end{array}$ & $\begin{array}{l}\text { 占 } \\
\hat{n} \\
m\end{array}$ & 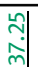 & $\begin{array}{l}\text { 尚 } \\
\text { ○े }\end{array}$ & $\begin{array}{l}0 \\
\text { D. } \\
\sim\end{array}$ & $\begin{array}{l}0 \\
0 \\
\text { ôm. }\end{array}$ & $\begin{array}{l}\tilde{\alpha} \\
\grave{m} \\
\text { (n) }\end{array}$ & $\begin{array}{l}\tilde{m} \\
\text { ָे }\end{array}$ & $\begin{array}{l}\vec{n} \\
\infty \\
\infty \\
m\end{array}$ & 윙 & 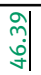 & 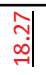 & $\begin{array}{l}\text { 岕 } \\
\text { ஸे }\end{array}$ & $\begin{array}{l}\overrightarrow{0} \\
\vdots \\
\vdots\end{array} \mid$ \\
\hline $\begin{array}{l}\text { PTC } \\
\text {. }\end{array}$ & $\begin{array}{l}\stackrel{p}{\sim} \\
\stackrel{\sim}{v}\end{array}$ & $\begin{array}{l}\tilde{n} \\
\stackrel{\lambda}{\vec{n}}\end{array}$ & $\begin{array}{l}\tilde{\alpha} \\
\infty \\
\sim\end{array}$ & $\begin{array}{l}\text { ㄱ: } \\
\text { : }\end{array}$ & \begin{tabular}{l}
$\stackrel{8}{\circ}$ \\
\multirow{\sim}{*}{}
\end{tabular} & $\begin{array}{l}m \\
\stackrel{m}{a} \\
\end{array}$ & 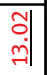 & $\begin{array}{l}\infty \\
0 \\
0 \\
\end{array}$ & $\begin{array}{l}\text { aे } \\
\dot{\theta}\end{array}$ & $\begin{array}{l}\text { 움 } \\
\text { in }\end{array}$ & $\begin{array}{l}\text { 离 } \\
\stackrel{\sim}{\sim}\end{array}$ & $\begin{array}{l}\hat{\lambda} \\
\dot{\sigma} \\
0\end{array}$ & $\begin{array}{l}\stackrel{\leftrightarrow}{+} \\
\stackrel{+}{A}\end{array}$ & 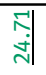 & $\begin{array}{l}\infty \\
\infty \\
\infty \\
0 \\
\end{array}$ & 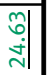 \\
\hline INF & 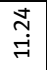 & $\vec{N}$ & $\begin{array}{l}\hat{\alpha} \\
\hat{m} \\
\dot{\eta}\end{array}$ & $\begin{array}{l}\text { ने } \\
\vec{b} \\
\rightarrow-1\end{array}$ & $\begin{array}{l}\stackrel{\circ}{a} \\
\text { mे }\end{array}$ & $\begin{array}{l}\hat{n} \\
\stackrel{-}{-1}\end{array}$ & $\begin{array}{l}\hat{0} \\
\dot{\theta}\end{array}$ & $\begin{array}{l}\infty \\
\stackrel{\infty}{\infty} \\
\infty\end{array}$ & 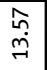 & $\begin{array}{l}\stackrel{p}{2} \\
a\end{array}$ & $\begin{array}{l}\stackrel{\sim}{\sim} \\
\infty \\
\infty\end{array}$ & $\begin{array}{l}m \\
a \\
o \\
0\end{array}$ & $\begin{array}{l}\text { ने } \\
\text { Oे }\end{array}$ & 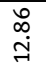 & $\begin{array}{l}\text { 年 } \\
\stackrel{-}{-}\end{array}$ & \begin{tabular}{l}
$\vec{F}$ \\
\multirow{a}{*}{}
\end{tabular} \\
\hline SUB & $\begin{array}{l}\text { Lo } \\
\dot{-}\end{array}$ & 윙 & $\begin{array}{l}\stackrel{2}{2} \\
\sigma\end{array}$ & $\begin{array}{l}\tilde{O} \\
\dot{a}\end{array}$ & $\begin{array}{l}\text { ô } \\
\text { gे }\end{array}$ & $\begin{array}{l}0 \\
\infty \\
\dot{\omega} \\
\dot{\sigma}\end{array}$ & $\begin{array}{l}m \\
\stackrel{m}{a} \\
\end{array}$ & $\begin{array}{l}\text { ָे } \\
\stackrel{\sim}{+}\end{array}$ & $\begin{array}{l}\stackrel{m}{i} \\
\underset{ }{\mid}\end{array}$ & $\begin{array}{l}\text { no } \\
\dot{-}\end{array}$ & $\begin{array}{l}\infty \\
\infty \\
a\end{array}$ & 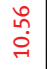 & $\begin{array}{l}\hat{N} \\
a\end{array}$ & $\begin{array}{l}\text { הֶ } \\
\text { }\end{array}$ & $\begin{array}{l}\overrightarrow{1} \\
b \\
\vec{b}\end{array}$ & $\begin{array}{l}\vec{n} \\
\ddot{\eta}\end{array}$ \\
\hline IND & 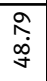 & ปี & $\begin{array}{l}\hat{n} \\
\tilde{n} \\
\stackrel{n}{n}\end{array}$ & \begin{tabular}{l}
$\sqrt{5}$ \\
\multirow{4}{*}{}
\end{tabular} & $\begin{array}{l}\vec{D} \\
\dot{b} \\
\stackrel{n}{n}\end{array}$ & $\begin{array}{l}\vec{b} \\
\stackrel{-1}{n}\end{array}$ & $\begin{array}{l}\overrightarrow{7} \\
\text { 呙 } \\
\text { n. }\end{array}$ & \begin{tabular}{l|}
$\overrightarrow{0}$ \\
$\overrightarrow{0}$ \\
\end{tabular} & 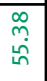 & वैं & $\begin{array}{l}\hat{\infty} \\
\infty \\
\infty \\
n\end{array}$ & 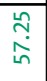 & 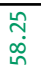 & 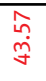 & $\begin{array}{l}\text { \& } \\
\text { in }\end{array}$ & $\begin{array}{l} \\
\dot{0} \\
\dot{\sigma}\end{array}$ \\
\hline × & वँّ & 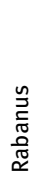 & 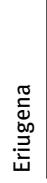 & $\begin{array}{l}\frac{E}{D} \\
\stackrel{n}{<} \\
\frac{5}{<}\end{array}$ & $\begin{array}{l}\frac{0}{0} \\
\frac{\pi}{0} \\
\frac{0}{\alpha}\end{array}$ & 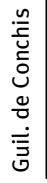 & 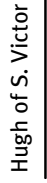 & 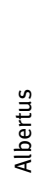 & 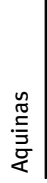 & 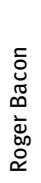 & $\frac{\mathfrak{n}}{\bar{\Xi}}$ & 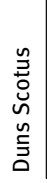 & $\begin{array}{l}\frac{E}{\pi} \\
\frac{\tilde{J}}{\frac{5}{\zeta}}\end{array}$ & 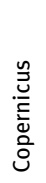 & $\begin{array}{l}\frac{n}{2} \\
\frac{1}{\pi} \\
\frac{\pi}{2} \\
0\end{array}$ & 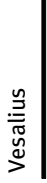 \\
\hline
\end{tabular}




\begin{tabular}{|c|c|c|c|c|c|c|c|c|c|c|c|c|c|c|c|c|c|}
\hline Entropy & $\begin{array}{l}\infty \\
\text { of } \\
\text { ol }\end{array}$ & $\begin{array}{l}\infty \\
\infty \\
\infty \\
\infty\end{array}$ & ָ̊ & $\begin{array}{l}\stackrel{n}{n} \\
\infty^{0}\end{array}$ & 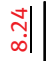 & $\begin{array}{l}\hat{n} \\
\dot{\sigma} \\
\sigma\end{array}$ & $\begin{array}{c}\widehat{c} \\
\text { co } \\
\infty\end{array}$ & $\begin{array}{l}a \\
\stackrel{a}{0}\end{array}$ & 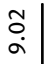 & $\begin{array}{l}\text { D. } \\
\infty \\
\infty\end{array}$ & $\begin{array}{l}0 \\
0 \\
0\end{array}$ & 윙 & $\begin{array}{l}0 \\
0 \\
0 \\
0\end{array}$ & $\stackrel{a}{\wedge} \mid$ & $\begin{array}{l}\stackrel{N}{N} \\
\sigma\end{array}$ & 윗| & $\stackrel{\text { จे }}{\text { I }}$ \\
\hline ABL ABS & $\begin{array}{l}\stackrel{0}{ } \\
\text { i }\end{array}$ & $\underset{m}{m}$ & 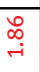 & $\begin{array}{l}\infty \\
\infty \\
\stackrel{\sim}{\rightarrow}\end{array}$ & $\begin{array}{l}\text { b्| } \\
\text { | }\end{array}$ & $\begin{array}{l}\stackrel{\sim}{\sim} \\
\underset{\sigma}{\sigma}\end{array}$ & 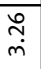 & \begin{tabular}{|c|c|} 
\\
|
\end{tabular} & 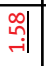 & 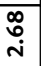 & $\begin{array}{ll}5 \\
3\end{array}$ & 齐 & 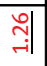 & 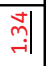 & $\stackrel{\substack{\hat{n} \\
\sim}}{ }$ & 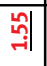 & $\begin{array}{l}0 \\
: \\
0\end{array}$ \\
\hline Modifiers & 今े & ํํㅇ & $\begin{array}{l}\underset{\sim}{\sim} \\
\text { त्र }\end{array}$ & 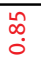 & $\underset{\rightarrow}{m}$ & $\begin{array}{l}\stackrel{p}{o} \\
\dot{0}\end{array}$ & 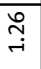 & $\begin{array}{l}\infty \\
\infty \\
0 \\
0\end{array}$ & 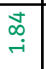 & $\begin{array}{l} \\
\infty \\
\infty \\
\rightarrow+\end{array}$ & \begin{tabular}{|c|}
$\overrightarrow{0}$ \\
0 \\
0
\end{tabular} & ભે & $\begin{array}{l}\infty \\
\stackrel{\sim}{\sim} \\
\end{array}$ & 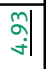 & $\underset{\sim}{m}$ & ఫેl & $\underset{i}{N}$ \\
\hline $\mathrm{N}$-SUF & $\begin{array}{l}\text { 於 } \\
\dot{b}\end{array}$ & 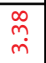 & $\underset{\sim}{\stackrel{n}{n}}$ & $\begin{array}{l}\stackrel{2}{i} \\
\text { in }\end{array}$ & ㄱํํ| & $\begin{array}{l}\mathcal{H} \\
\dot{m}\end{array}$ & 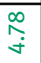 & $\begin{array}{l}\text { fे } \\
\text { in }\end{array}$ & 育 & 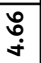 & 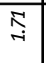 & 멕 & 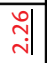 & 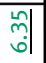 & $\begin{array}{l}\vec{r} \\
\text { m. } \\
\dot{\omega n}\end{array}$ & $\begin{array}{c}\hat{\alpha} \\
\dot{m}\end{array}$ & $\begin{array}{l}\bar{N} \\
\text {. }\end{array}$ \\
\hline ADJ-SUF & 겍| & $\begin{array}{l}\text { Na } \\
\text { N }\end{array}$ & 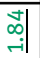 & $\begin{array}{l}\underset{\infty}{\rightarrow} \\
\rightarrow\end{array}$ & \begin{tabular}{l|} 
\\
영 \\
$\vdots$
\end{tabular} & 웍 & స్ & $\begin{array}{l} \\
\stackrel{\infty}{\sim} \\
\rightarrow\end{array}$ & 出 & $\begin{array}{l}\text { : } \\
\text { : }\end{array}$ & $\begin{array}{l}\hat{O} \\
\hat{0}\end{array}$ & î & 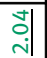 & $\vec{m}$ & ㅇำ & 이 & $\begin{array}{l}n \hat{n} \\
0 \\
0\end{array}$ \\
\hline 3rd PAS & 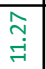 & 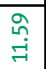 & $\begin{array}{l}\stackrel{\circ}{\infty} \\
\infty \\
\infty\end{array}$ & $\begin{array}{l}m \\
\infty \\
\stackrel{-}{r} \\
\end{array}$ & 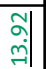 & 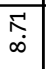 & 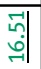 & 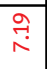 & 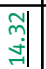 & \begin{tabular}{l} 
مी \\
\multirow{2}{*}{}
\end{tabular} & \begin{tabular}{l}
\multirow{\infty}{*}{} \\
$\stackrel{1}{ }$
\end{tabular} & $\begin{array}{l} \pm \\
\alpha \\
\alpha\end{array}$ & $\begin{array}{l}\text { 定 } \\
\infty\end{array}$ & \begin{tabular}{l|l} 
& \\
0 \\
$\mathfrak{d}$
\end{tabular} \mid & $\begin{array}{l}\circ \\
\stackrel{0}{0} \\
\stackrel{+}{r}\end{array}$ & $\begin{array}{l}\overrightarrow{0} \\
\dot{H}\end{array}$ & $\underset{\sim}{\overrightarrow{7}}$ \\
\hline 1st SG & 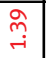 & बे & $\stackrel{\vec{m}}{\vec{m}}$ & 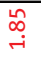 & $\begin{array}{l}\overrightarrow{0} \\
\dot{m}\end{array}$ & $\begin{array}{l}0 \\
\infty \\
\dot{m} \\
m\end{array}$ & $\widehat{\grave{\alpha}}$ & $\begin{array}{l}\infty \\
\infty \\
\infty \\
\vdots\end{array}$ & $\begin{array}{l}\text { 号 } \\
\stackrel{m}{m}\end{array}$ & $\begin{array}{l}\text { O̦ } \\
\text { N. }\end{array}$ & $\begin{array}{l}n \\
\stackrel{n}{n} \\
\tilde{N}\end{array}$ & 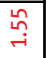 & $\begin{array}{l} \\
m \\
0\end{array}$ & \begin{tabular}{l}
$\stackrel{\text { \} }{\rightarrow}} \\
{ }$ & 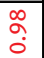 & 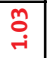 & वें \\
\hline PRON:POSS & 尚 & वी & a! & :ै। & \begin{tabular}{|c|}
$\overrightarrow{0}$ \\
0 \\
0
\end{tabular} & 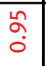 & गे। & $\begin{array}{l}0 \\
0 \\
0\end{array}$ & $\begin{array}{l}0 \\
0 \\
0 \\
0\end{array}$ & 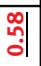 & $\begin{array}{l}\text { กิ } \\
\end{array}$ & $\begin{array}{l}0 \\
0 \\
0\end{array}$ & 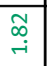 & \begin{tabular}{l|l} 
& 0 \\
0 & 1
\end{tabular} & $\begin{array}{l}0 \\
0 \\
0\end{array}$ & $\begin{array}{ll} \\
0 \\
0\end{array}$ & $\begin{array}{l}\infty \\
\stackrel{\infty}{\infty} \\
0\end{array}$ \\
\hline CONJ:S & $\begin{array}{l}\infty \\
\infty \\
\infty \\
m\end{array}$ & $\begin{array}{l}\stackrel{8}{\circ} \\
\text { i }\end{array}$ & $\begin{array}{l}\text { oे } \\
\stackrel{N}{N}\end{array}$ & $\begin{array}{l}\tilde{m} \\
\dot{m}\end{array}$ & $\begin{array}{l}\stackrel{N}{N} \\
\end{array}$ & $\begin{array}{l}\text { Ni } \\
\text { - }\end{array}$ & $\begin{array}{l}\stackrel{n}{\infty} \\
\dot{m}\end{array}$ & 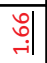 & $\begin{array}{l} \\
\\
i \\
N\end{array}$ & $\begin{array}{l} \\
\dot{m}\end{array}$ & कू & 윔 & $\begin{array}{l}\text { 윅 } \\
\text { f }\end{array}$ & $\begin{array}{l}0 \\
\vdots \\
\text { m.l }\end{array}$ & $\stackrel{\stackrel{\sim}{\sim}}{i}$ & $\begin{array}{l}\stackrel{P}{0} \\
\dot{m}\end{array}$ & aे \\
\hline$\overline{\text { REL }}$ & $\begin{array}{l}\stackrel{a}{\infty} \\
\stackrel{\sim}{i}\end{array}$ & $\underset{\sim}{\stackrel{N}{N}}$ & 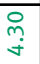 & 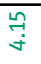 & 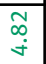 & $\begin{array}{l}\infty \\
\infty \\
\dot{m} \\
\dot{m}\end{array}$ & $\begin{array}{l}\vec{b} \\
\stackrel{\sim}{0}\end{array}$ & $\stackrel{\substack{n \\
\sim}}{i}$ & $\vec{m}$ & $\begin{array}{l} \pm \\
\text { mे }\end{array}$ & $\begin{array}{l} \\
\end{array}$ & $\underset{f}{\mathbb{f}}$ & $\begin{array}{l}\hat{m} \\
\dot{\sigma} \\
\dot{0}\end{array}$ & $\begin{array}{l}\underset{f}{\sim} \\
\underset{J}{*}\end{array}$ & 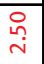 & $\begin{array}{l}\underline{n} \\
\dot{y}\end{array}$ & $\begin{array}{l}n \\
\stackrel{n}{r}\end{array}$ \\
\hline ESSE & 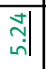 & $\begin{array}{l}\overrightarrow{0} \\
\dot{m}\end{array}$ & 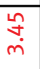 & $\begin{array}{l}\stackrel{\circ}{b} \\
\dot{m}\end{array}$ & 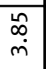 & 귕 & $\vec{\sigma}$ & 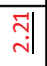 & 엥 & 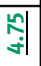 & $\begin{array}{l}\underset{\infty}{\infty} \\
\underset{\sim}{*}\end{array}$ & 규: & 임 & $\begin{array}{l}0 \\
0 \\
0 \\
0\end{array}$ & 워 & 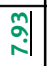 & $\stackrel{F}{\tilde{m}}$ \\
\hline ABL/DAT & $\begin{array}{l}\vec{F} \\
\text { ปें }\end{array}$ & 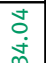 & $\begin{array}{l}\dot{8} \\
\dot{m}\end{array}$ & $\begin{array}{l}\infty \\
\stackrel{\infty}{\infty} \\
\infty \\
\sim\end{array}$ & 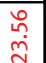 & $\begin{array}{l} \\
\infty \\
m \\
m\end{array}$ & 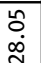 & $\begin{array}{l}\stackrel{\sim}{\tilde{j}} \\
\dot{m}\end{array}$ & $\begin{array}{l}\hat{o} \\
\dot{1} \\
\dot{v}\end{array}$ & 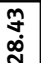 & \begin{tabular}{l|}
\multirow{b}{*}{} \\
$\dot{m}$ \\
$m$
\end{tabular} & 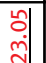 & 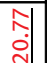 & 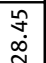 & $\begin{array}{l}\stackrel{\sim}{\infty} \\
\stackrel{\infty}{\sim}\end{array}$ & $\begin{array}{l}\text { 옴 } \\
\text { ஸे }\end{array}$ & $\begin{array}{l}\bar{D} \\
\dot{m} \\
\dot{m}\end{array}$ \\
\hline $\mathrm{ACC}$ & 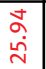 & $\begin{array}{l}\tilde{N} \\
\stackrel{N}{ }\end{array}$ & $\begin{array}{l}\stackrel{\sim}{\sim} \\
\stackrel{\sim}{N}\end{array}$ & $\begin{array}{l}m \\
0 \\
\infty \\
\sim \\
\sim\end{array}$ & 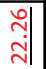 & 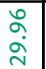 & 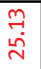 & $\begin{array}{l}\infty \\
\stackrel{\omega}{\sim} \\
\stackrel{\sim}{N}\end{array}$ & $\begin{array}{ll}\text { y } \\
\text { ஸे }\end{array}$ & $\begin{array}{l}\infty \\
\infty \\
\\
\text { ஸे }\end{array}$ & \begin{tabular}{l|}
$\bar{m}$ \\
$m$ \\
$m$
\end{tabular} & \begin{tabular}{l|} 
ते \\
\multirow{2}{*}{}
\end{tabular} & $\begin{array}{l}\begin{array}{l}n \\
\hat{D} \\
\dot{\sim}\end{array} \\
\end{array}$ & 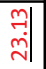 & $\begin{array}{l}\infty \\
0 \\
0 \\
\stackrel{m}{0}\end{array}$ & $\begin{array}{l}\overrightarrow{1} \\
\text { ำ }\end{array}$ & 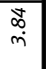 \\
\hline$\overline{\text { GEN }}$ & $\begin{array}{l}\infty \\
\stackrel{\infty}{0} \\
\dot{b} \\
\end{array}$ & 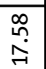 & 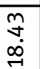 & $\begin{array}{l}\stackrel{0}{\circ} \\
\stackrel{\sim}{\sim}\end{array}$ & 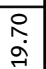 & 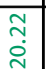 & 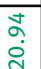 & 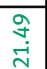 & 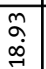 & $\begin{array}{l}\tilde{\infty} \\
\stackrel{\infty}{A}\end{array}$ & $\begin{array}{l}\stackrel{\infty}{\sim} \\
\text { n}\end{array}$ & \begin{tabular}{l|} 
\\
0 \\
0
\end{tabular} & $\begin{array}{l}\text { m̂n } \\
\text { ?. }\end{array}$ & $\begin{array}{l}0 \\
\stackrel{n}{0} \\
0\end{array}$ & $\begin{array}{l}\stackrel{n}{\stackrel{n}{\approx}} \\
\end{array}$ & $\begin{array}{l}\text { 원 } \\
\text { ज्ञl }\end{array}$ & $\begin{array}{l}\infty \\
\stackrel{\infty}{0} \\
m\end{array}$ \\
\hline NOM & $\begin{array}{l}\text { नी } \\
\text { D. } \\
\sim\end{array}$ & $\begin{array}{l}1 \\
0 \\
0 \\
0\end{array}$ & 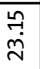 & 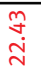 & $\begin{array}{l}\text { f } \\
\dot{m}\end{array}$ & \begin{tabular}{l|l|l|} 
\\
0 \\
0
\end{tabular} & $\begin{array}{l}\text { के } \\
\text { ஸे }\end{array}$ & \begin{tabular}{l} 
위 \\
\multirow{0}{*}{}
\end{tabular} & $\begin{array}{l}\text { वี } \\
\text { ஸे }\end{array}$ & 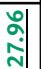 & 疋 & \begin{tabular}{l}
0 \\
$\infty$ \\
0 \\
\hdashline
\end{tabular} & $\begin{array}{l}\text { ज्: } \\
\text { ते }\end{array}$ & $\begin{array}{l}a \\
0 \\
\hat{m}\end{array}$ & $\begin{array}{l}\stackrel{m}{\sim} \\
\stackrel{\sim}{\sim}\end{array}$ & 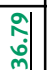 & \begin{tabular}{l}
$\infty$ \\
\multirow{2}{\alpha}{} \\
$\alpha$
\end{tabular} \\
\hline PTC & 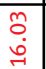 & 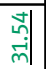 & 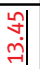 & $\begin{array}{l}\tilde{\alpha} \\
\text { ڤे }\end{array}$ & \begin{tabular}{l|} 
\\
0 \\
0 \\
0
\end{tabular} & 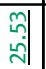 & 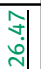 & 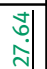 & \begin{tabular}{l|}
$\vec{f}$ \\
$\dot{0}$ \\
$\overrightarrow{-1}$
\end{tabular} & 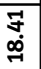 & $\begin{array}{l}\text { जे } \\
\text { जी }\end{array}$ & 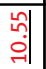 & $\begin{array}{l}\text { N̂ } \\
\stackrel{-}{N}\end{array}$ & 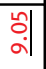 & $\begin{array}{l}\overrightarrow{0} \\
\stackrel{\sim}{N}\end{array}$ & 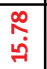 & वें \\
\hline INF & 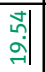 & $\begin{array}{c}\hat{m} \\
\infty\end{array}$ & 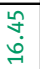 & $\begin{array}{l}\overrightarrow{0} \\
\infty \\
\infty \\
\sim\end{array}$ & 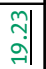 & \begin{tabular}{l} 
\\
\multirow{J}{J}{} \\
$\stackrel{y}{*}$
\end{tabular} & 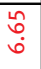 & $\begin{array}{l}\vec{i} \\
0 \\
0\end{array}$ & 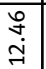 & 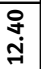 & 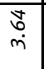 & $\begin{array}{l}\infty \\
\infty \\
\dot{\sim} \\
\dot{\sim}\end{array}$ & 斊 & $\begin{array}{l}\stackrel{n}{\sim} \\
\stackrel{7}{7}\end{array}$ & $\begin{array}{l}\stackrel{\text { }}{\sim} \\
\underset{H}{\forall}\end{array}$ & 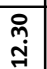 & $\begin{array}{l}\vec{y} \\
\text { in }\end{array}$ \\
\hline SUB & 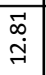 & $\begin{array}{l}\infty \\
\stackrel{0}{ } \\
\vdots \\
0\end{array}$ & $\begin{array}{l}\infty \\
\stackrel{0}{0} \\
\stackrel{1}{1}\end{array}$ & \begin{tabular}{l|l} 
& \multicolumn{1}{|c}{} \\
0 \\
$\vdots$ \\
$\vdots$
\end{tabular} & \begin{tabular}{l} 
\\
\multirow{J}{0}{} \\
$\stackrel{\ddots}{0}$
\end{tabular} & 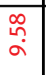 & $\begin{array}{l}m \\
\stackrel{\infty}{a} \\
\stackrel{\sim}{\sim}\end{array}$ & $\begin{array}{l}\infty \\
m \\
\omega^{\infty}\end{array}$ & $\begin{array}{l}\vec{t} \\
\stackrel{m}{H} \\
\vec{H}\end{array}$ & న్ ָి & 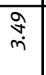 & 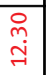 & $\begin{array}{l}\text { जे } \\
\text { जी }\end{array}$ & $\begin{array}{l}\stackrel{0}{1} \\
\stackrel{\text { In }}{ }\end{array}$ & 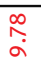 & $\begin{array}{l}0 \\
0 \\
\dot{9}\end{array}$ & $\begin{array}{c}\bar{m} \\
m\end{array}$ \\
\hline IND & 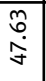 & $\begin{array}{l}m \\
\hat{n} \\
b \\
\forall\end{array}$ & $\begin{array}{l}8 \\
\text { aे } \\
\dot{q}\end{array}$ & \begin{tabular}{l|} 
\\
0 \\
0 \\
0 \\
0
\end{tabular} & 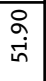 & \begin{tabular}{l} 
aे \\
bे \\
\multirow{y}{*}{}
\end{tabular} & $\begin{array}{l}\text { ণे } \\
\text { ซे }\end{array}$ & $\begin{array}{l}\tilde{\sigma} \\
\stackrel{\sigma}{\sigma}\end{array}$ & $\begin{array}{l}\stackrel{m}{\sim} \\
\stackrel{n}{n}\end{array}$ & जै & $\begin{array}{l}\text { बुे } \\
\text { के }\end{array}$ & 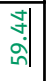 & 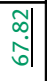 & 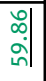 & $\stackrel{\tilde{N}}{i}$ & 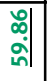 & $\begin{array}{l}m \\
\substack{n \\
b}\end{array}$ \\
\hline × & 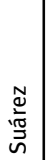 & 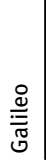 & 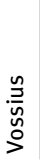 & 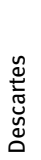 & $\begin{array}{l}\frac{N}{0} \\
\text { : } \\
\text { in }\end{array}$ & 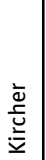 & 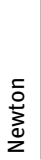 & 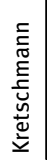 & $\begin{array}{l}\bar{\Phi} \\
\overline{0} \\
\bar{\infty}\end{array}$ & $\sum_{\substack{\infty \\
0}}^{\infty}$ & $\overrightarrow{\tilde{v}}$ & 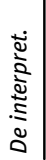 & $\begin{array}{l}\tilde{n} \\
\bar{s} \\
\overline{0} \\
\Sigma\end{array}$ & 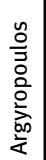 & 号 & 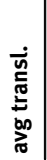 & छे \\
\hline
\end{tabular}
\end{tabular}




\begin{tabular}{|c|c|c|c|c|c|c|c|c|c|c|c|c|c|c|c|}
\hline Entropy & $\underset{N}{N}$ & : & $\begin{array}{l}\stackrel{m}{m} \\
a\end{array}$ & $\begin{array}{l}\infty \\
\infty \\
\infty \\
\infty\end{array}$ & ন্ & $\stackrel{?}{\stackrel{2}{a}}$ & $\stackrel{\widehat{N}}{a}$ & $\stackrel{2}{2}$ & ঙุ. & $\begin{array}{l}\circ \\
\infty \\
\infty\end{array}$ & $\begin{array}{l}\hat{\infty} \\
\infty \\
\infty\end{array}$ & $\begin{array}{l}\circ \\
b \\
\infty\end{array}$ & مૂ & ㅇํ & $\begin{array}{l}\infty \\
\stackrel{\infty}{a} \\
\stackrel{0}{0}\end{array}$ \\
\hline ABL ABS & 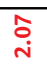 & 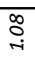 & 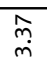 & 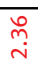 & $\stackrel{m}{\grave{v}}$ & 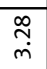 & $\underset{\sim}{\stackrel{N}{N}}$ & $\stackrel{m}{\dot{m}}$ & $\begin{array}{l}\overrightarrow{7} \\
0\end{array}$ & 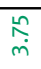 & ה્ઞ & $\begin{array}{l} \\
\stackrel{0}{+} \\
\dot{x}\end{array}$ & जे| & 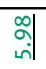 & $\begin{array}{l}\text { mे| } \\
\stackrel{y}{*}\end{array}$ \\
\hline Modifiers & $\underset{\sim}{N}$ & 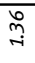 & $\underset{\sim}{\stackrel{n}{+}}$ & $\begin{array}{l}\widehat{\infty} \\
\overrightarrow{+} \\
+\end{array}$ & $\vec{\Re}$ & $\underset{\sim}{\stackrel{N}{+}}$ & $\begin{array}{l}\hat{\circ} \\
\stackrel{+}{1}\end{array}$ & 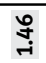 & 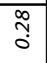 & $\stackrel{\text { 亗 }}{\rightarrow}$ & 구이 & 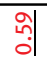 & $\begin{array}{l}\mid \overrightarrow{0} \\
\vdots \\
\vdots\end{array}$ & $\begin{array}{l}\text { ㅇํ. } \\
\text { مी }\end{array}$ & $\begin{array}{l}\text { مी } \\
0 \\
0\end{array}$ \\
\hline $\mathrm{N}$-SUF & $\begin{array}{l}\stackrel{0}{\circ} \\
\dot{j}\end{array}$ & $\stackrel{i}{i}$ & $\begin{array}{l}\stackrel{\bullet}{+} \\
\text { m. }\end{array}$ & $\begin{array}{l}\text { ฉ̆ } \\
\dot{m}\end{array}$ & 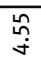 & 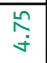 & $\underset{\sim}{\stackrel{J}{\sim}}$ & 강 & $\begin{array}{l}\overline{5} \\
0\end{array}$ & $\vec{g}$ & $\stackrel{\stackrel{\sim}{\alpha}}{\stackrel{\alpha}{\sigma}}$ & $\begin{array}{l}\tilde{D} \\
\stackrel{m}{m}\end{array}$ & $\begin{array}{c}0 \\
0 \\
0 \\
0\end{array}$ & 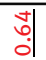 & $\begin{array}{l}0 \\
0 \\
0 \\
0\end{array}$ \\
\hline ADJ-SUF & 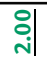 & $\stackrel{7}{7}$ & $\begin{array}{l}\hat{\circ} \\
\text { i }\end{array}$ & $\underset{+}{+}$ & $\underset{\sim}{\stackrel{m}{r}}$ & 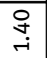 & $\stackrel{\substack{\infty \\
\sim}}{r}$ & $\stackrel{\sim}{\mathfrak{n}}$ & $\begin{array}{l}\infty \\
0 \\
0\end{array}$ & $\begin{array}{l}\hat{N} \\
0\end{array}$ & $\begin{array}{l}\text { of } \\
\text { o. }\end{array}$ & $\begin{array}{l}0 \\
\infty \\
\end{array}$ & $\stackrel{\stackrel{n}{m}}{\rightarrow}$ & $\underset{\sim}{\stackrel{\sim}{\sim}}$ & $\underset{\sim}{\sim}$ \\
\hline 3rd PAS & $\begin{array}{l}0 \\
\stackrel{0}{0} \\
\text { m. }\end{array}$ & $\underset{\forall}{\forall}$ & $\begin{array}{l}\stackrel{\leftrightarrow}{\stackrel{n}{\wedge}} \\
\end{array}$ & $\begin{array}{l}\text { no } \\
\text { o. }\end{array}$ & $\begin{array}{l}\stackrel{ \pm}{\prime} \\
\stackrel{-}{0}\end{array}$ & $\begin{array}{l}\infty \\
\stackrel{\infty}{ } \\
\alpha\end{array}$ & $\stackrel{\substack{n \\
\sigma}}{\sigma}$ & స: & बें & aे & of & क्षे| & 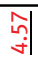 & స్ఫે & $\begin{array}{l}\infty \\
\stackrel{\infty}{\sim} \\
\stackrel{\sim}{\sim}\end{array}$ \\
\hline 1st SG & $\begin{array}{c}\infty \\
\rightarrow \\
\rightarrow\end{array}$ & I & $\begin{array}{l}\stackrel{N}{N} \\
\stackrel{n}{N}\end{array}$ & $\underset{\sim}{\mathcal{F}}$ & à & $\underset{m}{\stackrel{+}{m}}$ & $\begin{array}{l}\infty \\
\infty \\
m \\
m\end{array}$ & $\underset{J}{J}$ & స్ & ने & $\begin{array}{l}\tilde{O} \\
\text { Oे }\end{array}$ & 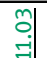 & 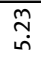 & $\stackrel{?}{?}$ & 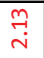 \\
\hline PRON:POSS & $\begin{array}{l}\vec{m} \\
\vdots\end{array}$ & $\stackrel{n}{n}$ & $\underset{\sim}{\stackrel{\sim}{r}}$ & $\stackrel{R}{\stackrel{R}{r}}$ & $\underset{\sim}{\stackrel{n}{\sim}}$ & $\underset{r}{\stackrel{9}{r}}$ & $\underset{\sim}{\stackrel{\sim}{\sim}}$ & นึ้ & $\bar{z}$ & f) & $\begin{array}{l}\checkmark \\
\text { d } \\
\text { S }\end{array}$ & $\underset{+}{\vec{r}}$ & 峁 & $\begin{array}{l}\text { \& } \\
\text { \& }\end{array}$ & 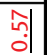 \\
\hline CONJ:S & 굴 & స్. & $\underset{\sim}{\tilde{N}}$ & $\vec{m}$ & $\begin{array}{l}\tilde{m} \\
\dot{m}\end{array}$ & ঐे & $\stackrel{\substack{n \\
N}}{N}$ & నू & $\begin{array}{l}\hat{n}_{n} \\
0\end{array}$ & $\begin{array}{l}\$ \\
\infty \\
0 \\
0\end{array}$ & 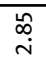 & 式 & 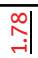 & 웜 & $\underset{\sim}{\stackrel{\sim}{\sim}}$ \\
\hline REL & 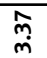 & $\begin{array}{l}0 \\
0 \\
0\end{array}$ & $\underset{\dot{J}}{\stackrel{J}{u}}$ & $\begin{array}{l}\vec{\infty} \\
\dot{m}\end{array}$ & $\begin{array}{l}\tilde{n} \\
\dot{m}\end{array}$ & $\begin{array}{l}\stackrel{\rho}{h} \\
\stackrel{n}{m}\end{array}$ & $\begin{array}{l}0 \\
\text { ర0 } \\
\text { in }\end{array}$ & $\stackrel{\substack{n \\
m}}{n}$ & $\begin{array}{l}\hat{n} \\
0\end{array}$ & 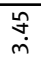 & $\begin{array}{l}\infty \\
\stackrel{m}{m}\end{array}$ & $\begin{array}{l}\text { I }_{\sim}^{\infty} \\
\stackrel{1}{*}\end{array}$ & 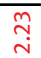 & $\begin{array}{l}\infty \\
\stackrel{\infty}{0} \\
\stackrel{N}{n}\end{array}$ & $\underset{\text { ì }}{\text { in }}$ \\
\hline ESSE & 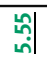 & $\underset{\sim}{\tilde{r}}$ & $\vec{a}$ & $\begin{array}{l}\infty \\
\stackrel{\infty}{+} \\
\dot{y}\end{array}$ & $\begin{array}{l}\stackrel{\circ}{m} \\
\stackrel{n}{n}\end{array}$ & $\underset{m}{\stackrel{f}{m}}$ & $\stackrel{\substack{\infty \\
\\
m}}{n}$ & $\underset{\dot{m}}{\mathbb{N}}$ & 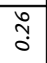 & 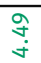 & 강 & $\stackrel{\sim}{n}$ & ঐे| & 离 & 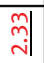 \\
\hline ABL/DAT & $\begin{array}{l}\underset{N}{N} \\
\text { م) }\end{array}$ & रे & $\begin{array}{l}\hat{\sim} \\
\stackrel{\sim}{v}\end{array}$ & 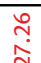 & $\begin{array}{l}\hat{n} \\
\infty \\
\infty\end{array}$ & 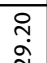 & స్ర & \ْ. & बे & $\begin{array}{l}\stackrel{N}{N} \\
\stackrel{N}{*}\end{array}$ & $\begin{array}{l}\text { ڤn } \\
\text { b. }\end{array}$ & $\begin{array}{l}0 \\
0 \\
0\end{array}$ & $\stackrel{1}{\infty}_{0}^{\infty}$ & $\begin{array}{l}\stackrel{\vec{f}}{\mathrm{~m}} \\
\end{array}$ & $\stackrel{2}{\dddot{2}}$ \\
\hline$\overline{A C C}$ & $\begin{array}{l}\infty \\
\text { m. } \\
\text { on }\end{array}$ & $\begin{array}{l}\hat{\omega}_{0} \\
\dot{\forall}\end{array}$ & $\begin{array}{l}\hat{\widehat{a}} \\
\mathrm{a}\end{array}$ & 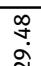 & 今. & 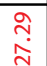 & 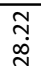 & $\begin{array}{l}f \\
\text { fo } \\
\infty\end{array}$ & $\underset{4}{4}$ & 寸) & ơ & $\begin{array}{l}0 \\
\text { m. } \\
\omega \\
\end{array}$ & م్ & $\begin{array}{l}\text { 님 } \\
\text { m. }\end{array}$ & $\begin{array}{l}n \\
\hat{n} \\
\dot{m}\end{array}$ \\
\hline GEN & $\begin{array}{l}\text { ฉ } \\
\text { ஸे }\end{array}$ & 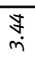 & $\begin{array}{l}\text { ڤn } \\
\sim \\
\sim\end{array}$ & 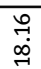 & $\begin{array}{l}\text { ¿̊ } \\
\text { : }\end{array}$ & $\begin{array}{l}\text { ڤ̊ } \\
\text { aे }\end{array}$ & $\begin{array}{l}\hat{n} \\
\infty \\
\sim\end{array}$ & $\begin{array}{c}\underset{\infty}{\infty} \\
\infty \\
\infty\end{array}$ & $\stackrel{n}{n}$ & $\begin{array}{l}\vec{f} \\
\underset{\sim}{\sim}\end{array}$ & 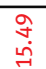 & $\begin{array}{l}\text { هั } \\
\text { స̊ }\end{array}$ & 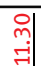 & $\begin{array}{l}m \\
m \\
0 \\
0\end{array}$ & $\begin{array}{l}\vec{y} \\
\dot{d}\end{array}$ \\
\hline NOM & $\begin{array}{l}\text { fol} \\
\text { pr| }\end{array}$ & 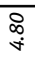 & $\begin{array}{l}\text { 命 } \\
\stackrel{\sim}{n}\end{array}$ & 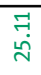 & $\begin{array}{l}\stackrel{m}{\infty} \\
\stackrel{\sim}{N}\end{array}$ & $\begin{array}{l}\text { ஃั } \\
\ddot{\sim}\end{array}$ & $\begin{array}{l}\hat{\text { ஸे }} \\
\text { N }\end{array}$ & $\begin{array}{l}\vec{n} \\
\stackrel{n}{n}\end{array}$ & $\underset{7}{\stackrel{2}{7}}$ & $\begin{array}{l}\overrightarrow{\hat{N}} \\
\ddot{\sim}\end{array}$ & $\begin{array}{l}\underset{\sim}{\sim} \\
\stackrel{\sim}{\sim}\end{array}$ & 웜 & $\begin{array}{l}\vec{m} \\
\stackrel{\sim}{N}\end{array}$ & 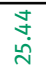 & $\begin{array}{l}\infty \\
0 \\
\stackrel{\sim}{d}\end{array}$ \\
\hline PTC & $\begin{array}{l}\stackrel{n}{\tilde{N}} \\
\stackrel{N}{N}\end{array}$ & $\begin{array}{l}\text { aे } \\
6\end{array}$ & $\begin{array}{l}\text { 욱 } \\
\text { ㄱ. }\end{array}$ & 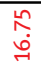 & $\begin{array}{l}\overrightarrow{7} \\
\text { ㄱ. }\end{array}$ & $\begin{array}{l}\hat{m} \\
\text { aे }\end{array}$ & $\begin{array}{l}\infty \\
\infty \\
\infty \\
\dot{N}\end{array}$ & $\begin{array}{l}\text { ț } \\
\text { ه̊ }\end{array}$ & 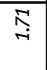 & $\begin{array}{l}\text { ్ָ } \\
\underset{\sim}{\sim}\end{array}$ & $\begin{array}{l}\text { రे } \\
\text { aे }\end{array}$ & $\begin{array}{l}\text { बे } \\
\dot{\vec{\lambda}}\end{array}$ & 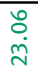 & $\begin{array}{l}\text { مू } \\
\vec{\lambda}\end{array}$ & 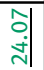 \\
\hline INF & $\begin{array}{l}\stackrel{9}{6} \\
6\end{array}$ & $\begin{array}{l}\text { जo } \\
\dot{m}\end{array}$ & $\begin{array}{l}m \\
m \\
\omega_{n}^{n}\end{array}$ & $\begin{array}{l}\text { वे } \\
\text { ᄋ్ }\end{array}$ & $\begin{array}{l}0 \\
\infty \\
0 \\
\dot{9}\end{array}$ & $\begin{array}{l}\stackrel{n}{1} \\
0 \\
0\end{array}$ & \begin{tabular}{l}
0 \\
$\stackrel{7}{d}$ \\
\multirow{d}{*}{}
\end{tabular} & $\begin{array}{l}\text { Iี } \\
\text { ปุ }\end{array}$ & $\stackrel{\vec{A}}{i}$ & $\begin{array}{l}\overrightarrow{+} \\
\vec{f}\end{array}$ & $\begin{array}{l}\infty \\
0 \\
0 \\
0\end{array}$ & 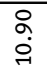 & $\begin{array}{l}\infty \\
\stackrel{\infty}{0} \\
\stackrel{\sim}{0}\end{array}$ & $\begin{array}{l}\infty \\
\infty \\
\alpha \\
\alpha\end{array}$ & $\begin{array}{l}\stackrel{\sim}{n} \\
\ddot{\sim}\end{array}$ \\
\hline SUB & 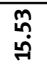 & in & $\begin{array}{l}\text { 움 } \\
\stackrel{-}{-}\end{array}$ & 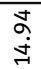 & $\stackrel{\stackrel{J}{m}}{\stackrel{m}{\rightarrow}}$ & $\begin{array}{l}\stackrel{9}{+} \\
\stackrel{+}{9}\end{array}$ & $\begin{array}{l}\hat{N} \\
\stackrel{ }{\sim}\end{array}$ & $\begin{array}{l}\vec{m} \\
\dot{\sim}\end{array}$ & $\underset{\sim}{n}$ & 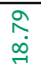 & $\begin{array}{l}\vec{b} \\
\dot{0}\end{array}$ & $\begin{array}{l}\text { No } \\
\infty \\
0 \\
0\end{array}$ & $\begin{array}{l}\text { ¿ } \\
\text { تُ }\end{array}$ & à & $\begin{array}{l}\hat{n} \\
\vec{f} \\
\vec{f}\end{array}$ \\
\hline IND & $\begin{array}{l}\overrightarrow{\tilde{n}} \\
\text { กิ }\end{array}$ & న్ & $\begin{array}{l}\infty \\
\stackrel{\infty}{ } \\
\dot{\gamma} \\
\dot{\gamma}\end{array}$ & $\begin{array}{l}\tilde{N} \\
\text { in } \\
\end{array}$ & $\begin{array}{l}\text { St } \\
\text { in }\end{array}$ & $\begin{array}{l}\overrightarrow{9} \\
\text { ก }\end{array}$ & 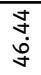 & $\begin{array}{l}\bar{m} \\
\stackrel{g}{\sigma}\end{array}$ & $\stackrel{\overrightarrow{9}}{m}$ & $\begin{array}{l}0 \\
\stackrel{9}{1} \\
0 \\
+\end{array}$ & 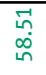 & $\begin{array}{l}\stackrel{\sim}{ } \\
\text { Nh}\end{array}$ & $\begin{array}{l}\hat{\sim} \\
\text { aे }\end{array}$ & $\begin{array}{l}\text { ㄱ. } \\
\stackrel{M}{n} \\
\text { nn }\end{array}$ & \begin{tabular}{l}
$\hat{n}$ \\
$\hat{\sigma}$ \\
\multirow{\sigma}{*}{}
\end{tabular} \\
\hline 区 & 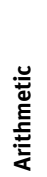 & $\frac{\overrightarrow{\tilde{z}}}{\vec{n}}$ & 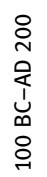 & 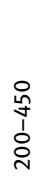 & $\begin{array}{l}8 \\
0 \\
i \\
\infty \\
\infty\end{array}$ & $\begin{array}{l}\stackrel{2}{ } \\
\underset{7}{1} \\
\stackrel{0}{0} \\
\stackrel{7}{7}\end{array}$ & $\begin{array}{l}0 \\
\text { D } \\
\stackrel{1}{1} \\
\stackrel{1}{0} \\
\stackrel{0}{n}\end{array}$ & $\sum_{\pi}^{\infty}$ & $\frac{\vec{v}}{\vec{n}}$ & 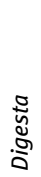 & 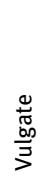 & 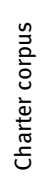 & 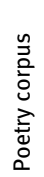 & 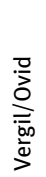 & 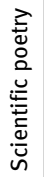 \\
\hline
\end{tabular}




\section{Characterising scientific Latin texts in contrast to other texts}

\$5 Tables 12 and 13 have presented numerical data about PoS and features that could be relevant for scientific Latin in light of what was reviewed (chap. 17) for scientific English. They show some traits rather clearly. In general, the composition of PoS differs very strongly between prose and poetry samples. All values for the poetry samples are outside 1 stdev of the benchmark prose samples: poetry has more ADJ, N, and V, but fewer CONJ, PREP, and PRON. Non-scientific poetry additionally has fewer ADV; scientific poetry is inconclusive in this respect. The charters show some traits typical for substandard Latin: high numbers of CONJ, PREP, but also a very nominal language (low V, high $\mathrm{N}$, high ADJ). The Vulgate shows some similar traits (high CONJ and PRON), as do the Digesta (high CONJ, though not subordinating ones; high V; low ADJ, ADV, PRON), although these two samples clearly represent very different types of Latin. Within scientific prose, the translations from Greek exhibit some common features (although they are translations of very different texts), in particular more CONJ and PRON, fewer N. This may represent general differences between Greek and Latin. The scientific sample has fewer V, N, and PRON, but more ADJ, ADV, and PREP. CONJ are more common than in the benchmark in many texts, but less so in others, producing an average value close to that of the benchmarks.

The more specific parameters in table 13 also show significant differences between the average of the five benchmark corpora on the one hand and poetry or translations from Greek on the other, and, less clearly, also differences between the scientific texts and the benchmark. Verbal and nominal subcategories (the first eight columns) produce less clear differences than the other parameters. Poetry has fewer SUB but more PTC (and to a lesser extent INF), fewer GEN but more NOM and ACC; the translations more NOM, fewer GEN, more IND, and surprisingly fewer PTC (although this depends strongly on the author). The scientific texts show less clear trends for these eight parameters on average: more NOM, fewer ACC and ABL/DAT. Only the amount of NOM differs strongly; this case is much more common. The verbal parameters are on average within 1 stdev of the five benchmark samples. But many of the individual texts are outside the normal range, some above, some below. The next chapter will try to find groups of scientific writing styles to match such cases.

The twelve more specifically chosen parameters differ more clearly. They were selected from a greater number of similar parameters, many of which did not differ markedly from the benchmark (somewhat surprising in the case of subordinating CONJ and word length, which are the only ones printed). Poetry tends to have lower numbers for all of the values shown, except ABL ABS and entropy, for which it has much higher values (ADJ-SUF, PRON:POSS, 1st SG are within normal range); the translations show quite the inverse behaviour (except 
REL). The scientific texts behave more or less like the translations from Greek, except that REL and 3rd PAS are also higher but subordinating CONJ are within range and N-SUF are lower. Parameters that differ strongly in both this scientific corpus and the arithmetic one (described in the next chapter, but average numbers are already included in the tables above) are ESSE, 3rd PAS, ADJ-SUF, and REL (all higher) and 1st SG, PRON:POSS, ABL ABS, and entropy (lower). As a first result, we may consider these values as significantly different in scientific Latin.

Among the three out-group corpora, not very surprisingly the charters often use the first person singular and the indicative as well as the ablative and especially the ablative absolute. If the many 'unknown' place and person names were included, the numbers of ABL might rise even further. They also use PTC more often than usual. The Digesta use SUB and INF conspicuously often; quite the opposite is true for the Vulgate, which on the other hand betrays its Greek origin by the high numbers of modifiers. Interestingly, suffixes are rare.

Among these data, a progression can often be observed: poetry - scientific poetry - general prose (five benchmark corpora) - the scientific texts - the five translations from Greek. Sometimes the arithmetic sample from chapter 20 below follows at the end, as can be seen in table 14 .

Table 14: Progressions for some values. Green marks the maximum value, red the minimum value (not including the arithmetic sample).

\begin{tabular}{|c|c|c|c|c|c|c|}
\hline & Poetry & $\begin{array}{r}\text { Scientific } \\
\text { poetry }\end{array}$ & 5 benchmarks & $\begin{array}{l}\text { Sample of } 40 \\
\text { science texts }\end{array}$ & $\begin{array}{r}\text { Translations } \\
\text { from Greek }\end{array}$ & $\begin{array}{l}\text { Arithmetic } \\
\text { (chap. 20) }\end{array}$ \\
\hline ADV & 7.2 & 8.5 & 9.0 & 9.7 & 13.0 & 8.3 \\
\hline CONJ & 7.3 & 8.0 & 9.9 & 9.9 & 12.0 & 10.2 \\
\hline $\mathrm{N}$ & 30.5 & 29.7 & 25.7 & 24.5 & 20.7 & 19.7 \\
\hline PREP & 4.4 & 5.3 & 8.3 & 9.1 & 8.9 & 11.1 \\
\hline V & 24.6 & 24.3 & 22.9 & 21.9 & 16.2 & 20.8 \\
\hline ESSE & 2.0 & 2.3 & 3.7 & 4.8 & 7.9 & 5.6 \\
\hline REL & 2.2 & 2.4 & 3.5 & 3.9 & 4.1 & 3.4 \\
\hline PRON:POSS & 1.5 & 0.6 & 1.6 & 0.6 & 0.7 & 0.3 \\
\hline 1st SG & 5.2 & 2.1 & 4.4 & 2.5 & 1.0 & 1.9 \\
\hline 3rd PAS & 4.6 & 7.7 & 9.1 & 12.8 & 11.0 & 13.6 \\
\hline ADJ-SUF & 1.4 & 1.2 & 1.3 & 2.0 & 3.0 & 2.0 \\
\hline N-SUF & 0.9 & 0.9 & 4.2 & 4.7 & 4.0 & 4.6 \\
\hline Modifiers & 0.6 & 0.8 & 1.5 & 1.9 & 3.0 & 2.2 \\
\hline
\end{tabular}


Table 14: (continued)

\begin{tabular}{l|r|r|r|r|r|r}
\hline & Poetry & $\begin{array}{r}\text { Scientific } \\
\text { poetry }\end{array}$ & 5 benchmarks & $\begin{array}{r}\text { Sample of 40 } \\
\text { science texts }\end{array}$ & $\begin{array}{r}\text { Translations } \\
\text { from Greek }\end{array}$ & $\begin{array}{r}\text { Arithmetic } \\
\text { (chap. 20) }\end{array}$ \\
\hline ABL ABS & 5.5 & 4.4 & 3.0 & 2.7 & 1.6 & 2.1 \\
\hline Entropy & 9.92 & 9.68 & 9.15 & 8.84 & 7.70 & 7.26 \\
\hline
\end{tabular}

For some parameters the scientific texts show extreme values (which would seem to be especially typical for scientific texts): PREP, PRON:POSS, 3rd PAS, N-SUF; often the arithmetic sample shows even more extreme values in these cases. The scientific poetry text sample tends to behave like other poetry. Only rarely does it behave more like other scientific texts; this is especially the case for PRON:POSS and 1st SG. The next chapter (chap. 19) will try to name subgroups in the general scientific corpus according to characteristic parameters and thus come back to types of Latin encountered in part 2 above.

$\$ 6$ Principal component analysis (PCA) ${ }^{40}$ is now applied to these data in order to plot them and to render these (or other) groups more clearly visible. The closer points are situated on the plot, the more similar these texts are in the parameters considered. The idea of PCA is to project multidimensional data onto two dimensions in the 'best' way, that is, preserving as much of the variation as possible. In order to achieve this, the $n$-dimensional data - for instance consisting of $n$ of the above parameters for each sample - is transformed orthogonally in the direction of the maximal variation within the data, producing the first dimension, the second dimension is the dimension with the highest variation under the constraint of being orthogonal to the first one (and so on, but usually only two dimensions are plotted). 'PC1' in the plots thus shows this first and most significant component; the accompanying value in the graphics the percentage of the entire variation; 'PC2' the second component. In our samples, these two 'best' dimensions together tend to represent between $60 \%$ and $70 \%$ of the entire variation, in other words only about two-thirds of the entire variation. The components themselves are made up of linear combinations of all $n$ parameters and, therefore, do not represent an intuitively meaningful quantity.

40 Clustvis (https://biit.cs.ut.ee/clustvis), a handy free online tool based on R, was used. The tool automatically turns values into standard scores (average 0 , stdev 1), so no prior calculation of these is required. 
The following plots depict the forty scientific texts (numbered chronologically) and those translated from Greek (blue) together with the five benchmark samples (green, boxed); the out-groups poetry (red), Digesta, charters, Vulgate (italics); and the corpora that will be studied in chapter 20 (arithmetic, historiography, scientific poetry, medicine, all in pink). Additionally, there are three groups of texts from within the forty texts named 'classical', 'scholastic', and 'new' science (also pink). ${ }^{41}$ They may represent especially typical subgroups. Despite the fact that Latin is (rightly) seen as a very homogeneous and stable 'fixed' language, clustering separates some major groups fairly well. The PoS work quite well for grouping, the values that were found to be specific for scientific texts even better. What we would expect from a 'good' clustering separating scientific texts from others is that

- the poetic out-group will be separated from the rest (and Vergil/Ovid close by);

- the Vulgate will also be separated from the rest, although it will be expected to influence some of the mediaeval scientific texts;

- the large prose benchmark samples will be reasonably close together, especially on the one hand the three from the Middle Ages and on the other hand possibly the classical and the Neo-Latin ones.

Of course, we also have some preconceptions from the scientific approaches and periods described in part 2 above: for instance, scholastic texts are expected to be grouped together.

First, a simple two-dimensional plot $\mathrm{N}$ against $\mathrm{V}$ is presented (fig. 34, without PCA). Such a simple plot can already separate the metrical texts quite well (top right), and puts the benchmark corpora approximately in the middle, the Greek translations in the bottom left, and the scholastic and arithmetic texts centre-left. The average value for all forty scientific texts in the sample ('avg 1-38') ${ }^{42}$ lies slightly to the left of the benchmark (indicating fewer nouns), close to the medical sample and to the Vulgate. The historiographical sample lies on the other side, toward the poetic texts. Some technical artes authors also group to the right (more N): especially Pliny, Rabanus, Vitruvius, Bede, Isidore. In general, it would seem that 'new' science texts use fewer $\mathrm{V}$, scholastic ones (unexpectedly) fewer $\mathrm{N}$, juridical texts (Digesta, Gaius, Pauli sententiae) more V, and at least Pauli sententiae more N.

A more sophisticated plot (fig. 35) for the same texts uses all seven PoS (ADJ, ADV, CONJ, N, PREP, PRON, V); the seven-dimensional data is optimally fitted to

41 They comprise respectively Varro, Cicero, Seneca; Albertus, Aquinas, Ockham, Suárez; Copernicus, Vesalius, Galileo, Newton.

42 A single number is used for the two Boethius texts and for the two juridical primary texts, so the numbering reaches only 38 , even though there are 40 texts. 


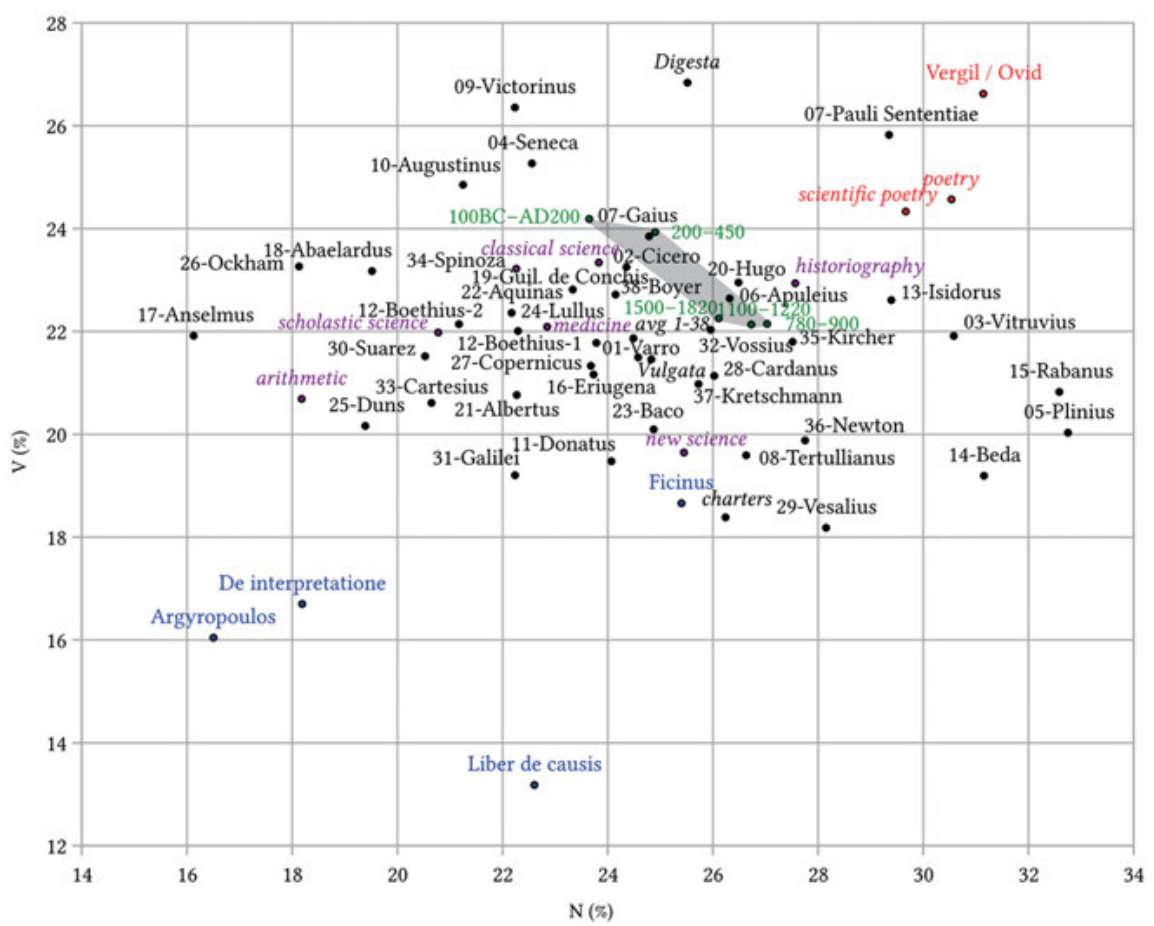

Fig. 34: Simple N vs V plot. Details in the main text.

two dimensions using PCA. Interestingly, the plot is quite similar to the simple $\mathrm{N}$ vs V plot. Again, it sets the poetical texts quite clearly apart from the rest. The translations from Greek, too, are on the other side of the plot. The scientific texts tend to be below ${ }^{43}$ the benchmark samples, though the juridical and the 'classical' ones do not. The scholastic ones are again more to the left, the technical ones and those of the Scientific Revolution to the right. All in all, these PoS results seems interesting, though not very distinctive for scientific Latin in general.

In a further plot (fig. 36), all eleven parameters introduced above as potentially typical for scientific Latin are used: ESSE, REL, CONJ:S, PRON:POSS, 1st SG, 3rd PAS, ADJ-SUF, N-SUF, modifiers, ABL ABS, entropy. The non-scientific texts are now much more clearly separated from most texts of the scientific sample; indeed, none of them are plotted within the greyed-out area enclosing the five non-

43 The coordinates in these plots are relative values without an intuitive meaning, so 'up', 'down', and similar terms are merely relative terms. The plots' orientation was chosen to be such that the translations from Greek were always at the bottom left in order to facilitate comparison. 


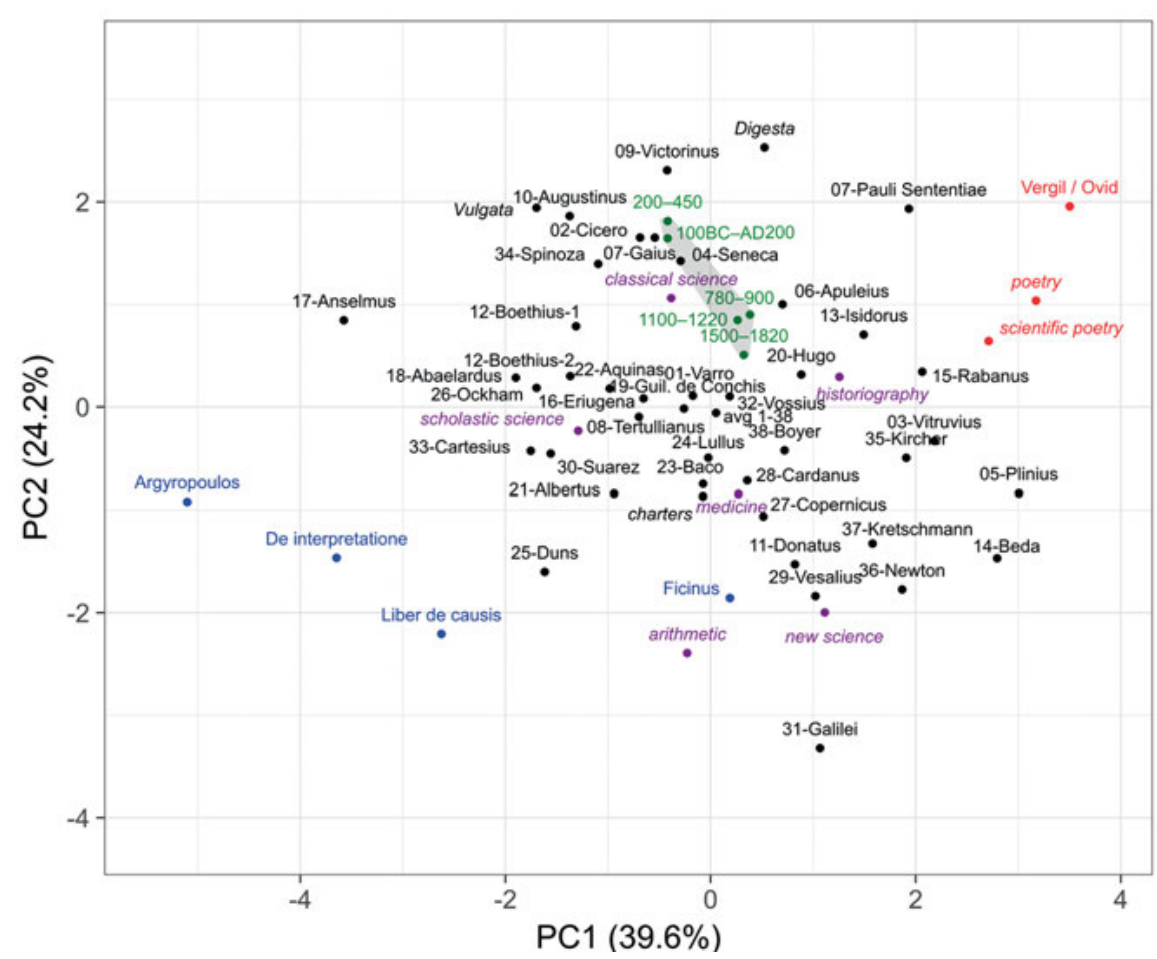

Fig. 35: PCA plot using the amounts of the seven PoS.

scientific benchmark samples. To the far left, the scholastic texts (including their 'father' Boethius) group quite neatly. Neo-scholastic ones (Suárez and Boyer) are between them and the centre of the plot. Many texts from Antiquity have a tendency to remain close to the five benchmark samples; in other words, their language is less distinctive than that of later scientific texts, for instance Varro, Cicero, Augustine, Seneca, Donatus. The two Neo-Latin human science texts (Kircher and Kretschmann), the charters, and historiography are located at the other side of the plot, close to the poetic texts. The translations from Greek are scattered all over the left-hand side of the plot, though the humanist translator Ficino ends up away from the others. Technical/encyclopaedic texts end up at the top centre (Vitruvius, Pliny, Bede, Isidore, Cardano, Vesalius, Galileo, Newton), the Vulgate at the bottom, far apart from the rest. The scientific corpus as a whole and the arithmetic corpus studied in the next chapter are situated in the centre together with some apparently 'inconspicuous' scientific texts: Gaius, Eriugena (surprisingly), Guilelmus de Conchis, Hugh of St Victor, and Roger Bacon, among others. Marius Victorinus, Anselm, and Lullus lean toward the translations from Greek. 


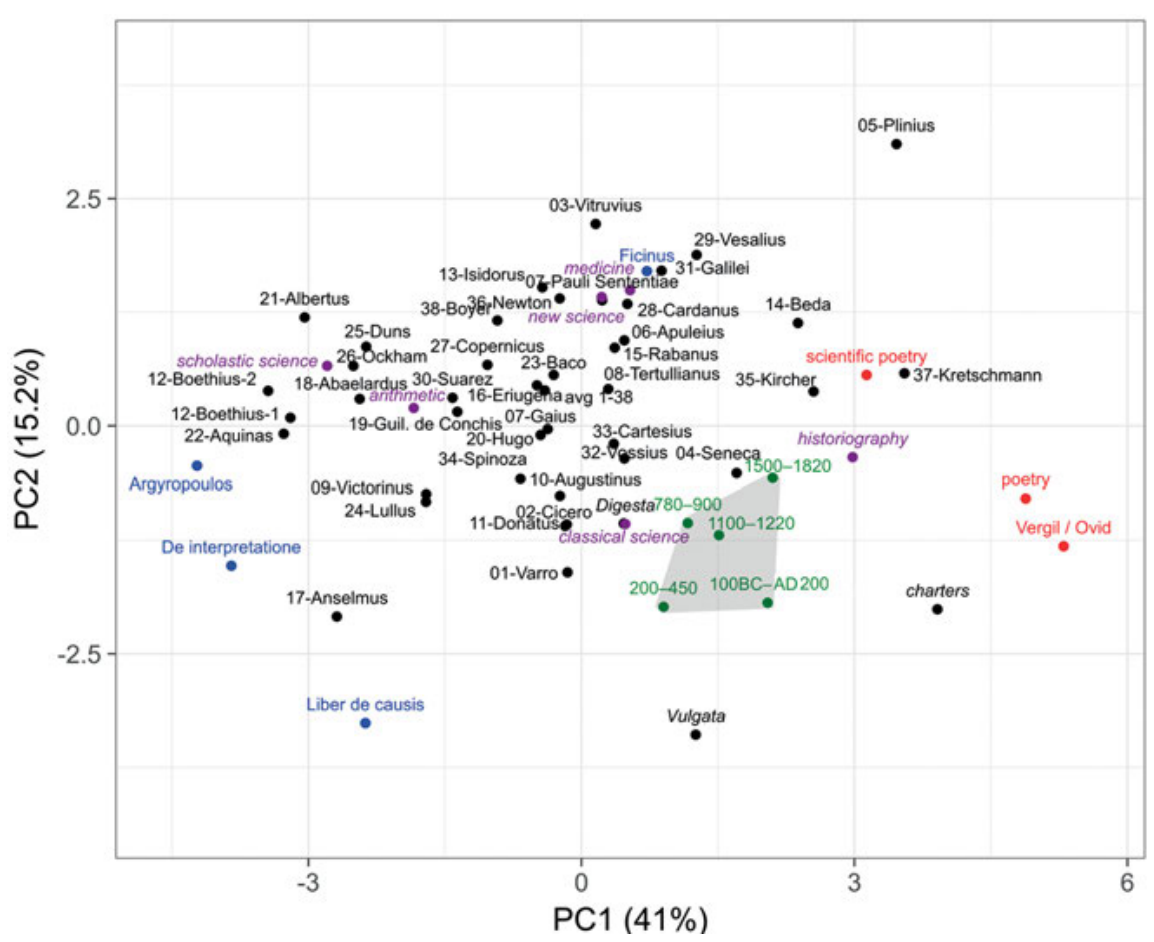

Fig. 36: PCA plot of all eleven parameters from the right half of table 13.

In the hope of getting an even neater result, now (fig. 37) only those parameters are used that differ at least 3 stdevs for the average of the forty scientific texts from the benchmark average. These are NOM, ESSE, PRON:POSS, 1st SG, ${ }^{44}$ 3rd PAS, ADJ-SUF. The five benchmark samples remain together (Varro, Cicero, Seneca, Augustine close to them); the poetry, Vulgate, and charter out-groups are far to the right. Technical texts together with texts from the Scientific Revolution are quite neatly apart from one another and from the benchmark, but it is the scholastic ones that are most clearly separated from the rest. Medicine and arithmetic are in between, medicine closer to the texts of the Scientific Revolution, arithmetic to the technical ones. Thus, the more practical or experimental sciences stay together, in contrast to the more theoretical ones. Again, early modern human

44 Obviously, 1st SG cannot be lower than 3 stdevs of $4.44 \pm 1.93$, which is due to the high stdev in the benchmark samples, which is again due to much higher amounts of 1st SG forms in the classical benchmark sample than the others. The scientific texts are in many cases very much lower (below 1), so including this value seemed warranted. 
scientists and historiographers are close to one another and the benchmark samples; the charters are now somewhat further off.

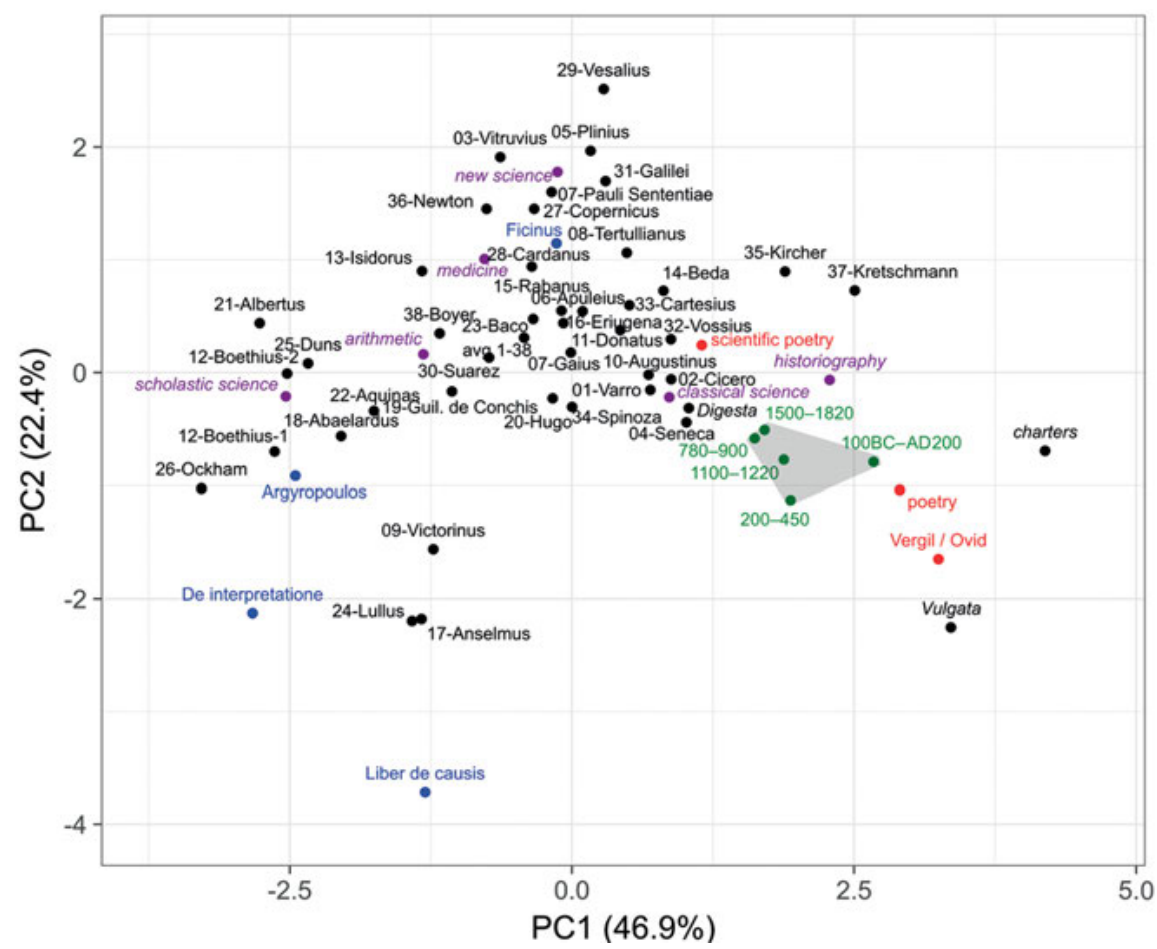

Fig. 37: PCA plot of the parameters for which the average of the scientific sample differs more than 3 stdevs from the benchmark: NOM, ESSE, PRON:POSS, 1st SG, 3rd PAS, ADJ-SUF.

For a final plot, the values whose averages in the forty scientific texts or in the arithmetic sample differ more than 3 stdevs from the benchmark were used (see fig. 38), a set of parameters that will be used again in chapter 20. These are ten parameters: ADJ, N, PREP, NOM, ESSE, PRON:POSS, 1st SG, 3rd PAS, ADJ-SUF, entropy. Many things remain the same as in the previous plot, but the groups of scholastic versus Scientific Revolution vs 'normal' texts become clearer (highlighted by green lines).$^{45}$ The arithmetic sample is now clearly within the scholastic group.

45 Moving the near-vertical line somewhat further right would include Guilelmus de Conches, Suárez, and Spinoza in the scholastic group. These authors do indeed seem to lie somewhere between scholastic and more 'normal' Latin. 
Scientific texts from Antiquity and human science texts tend to remain close to the benchmark samples. The Vulgate and the poetry samples separate clearly from the rest, but in another direction. In all plots, the juridical texts (Digesta, Gaius, Pauli sententiae) do not cluster close together. They belong to quite different genres, and it seems that jurisprudence - in Antiquity the most Roman of all sciences, as has been pointed out (chap. 8 \$12) - used heterogeneous language in different genres, at least more so than other sciences. In this plot, by far the main part of the information (40.6\%) lies on the $x$-axis; the $y$-axis displays only $19.7 \%$, not much more than the third, not depicted, dimension would. The most robust result is, therefore, certainly found on the $x$-axis; the scholastic texts are therefore the most distinct ones in the sample. ${ }^{46}$

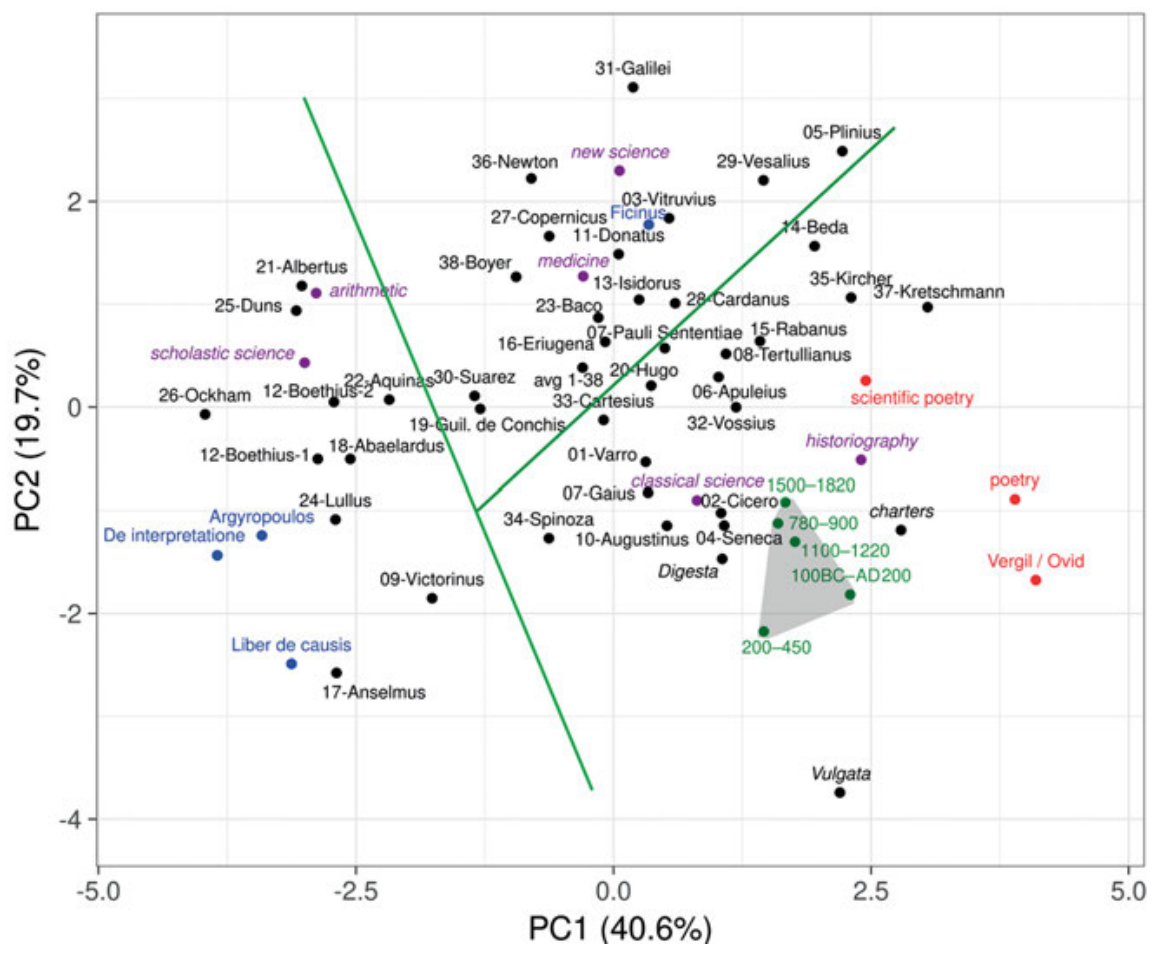

Fig. 38: PCA plot of the ten parameters for which the average of the scientific or the arithmetic sample (treated below, chap. 20) differs more than 3 stdevs from the benchmark: ADJ, N, PREP, NOM, ESSE, PRON:POSS, 1st SG, 3rd PAS, ADJ-SUF, entropy.

46 PC1's vector components: ADJ: 0.03, N: 0.39, PREP: -0.23, NOM: -0.42, ESSE: -0.44, PRON: POSS: 0.26 , 1st SG: 0.28 , 3rd PAS: -0.25 , ADJ-SUF: -0.21 , entropy: 0.43 . Thus, ADJ is the piece of information that contributes least, ESSE, NOM, and entropy the most. 
\$7 Another approach that can be used to group the texts is stylometry. Although it was conceived to solve questions of authorship, this method may also produce meaningful results for distinguishing scientific types of language. The most common $n$ tokens (words or lemmata) in each text are compared. ${ }^{47}$ Their frequencies are stored in a vector, and the distance between the vectors corresponding to the text samples is then calculated. ${ }^{48}$ The result is a clustered tree view based on a distance matrix calculated from the frequencies. This approach thus compares the core vocabulary of different texts. Figures 39 and 40 show a plot for the 100 most common words and lemmata respectively. In order to lessen the impact of specialised disciplines' specific vocabulary, a culling value of $80 \%$ was used, which means that only tokens present at least once in at least $80 \%$ of all samples (i.e. 46 of the 58 ones used) are taken into consideration. Tentative names for groups are superimposed on the plots.

The results are relatively similar to the previous PCA plots (bearing in mind that very different parameters are used). Some groups form more as would be expected in the word-based plot, some in the lemma-based one. The non-scientific metrical texts (red) cluster together in both plots, the benchmark samples only partly: the antique sample is found close to Cicero in both plots, not together with the other benchmark samples. The Vulgate and charters cluster together with the other benchmark samples as non-scientific texts. Scholastic texts tend to separate most neatly from the rest; they cluster together with the translations from Greek (except, again, Ficino). Strangely, Aquinas is found somewhere else in the lemma-based plot, the neo-scholastic author Boyer in both. The three juridical texts cluster together much better than in the PCA plots. In both plots, there are two groups comprising mostly natural-science authors and human-science authors respectively. In the word plot, Augustine and, surprisingly, Eriugena end up with the non-scientific texts. Changes in the sample size (i.e. adding or removing texts) can produce strange changes (not shown). Changing the number of tokens considered, on the other hand, leaves the result quite stable. In general, it would seem that the smaller units are usually relatively stable and tend to correspond well to expectations, but the uppermost bifurcations in the tree are not very

47 I used the stylo package for R (as described in Eder, Rybicki \& Kestemont 2016). 'Unknown' lemmata were, again, removed from the samples.

48 The simple and intuitive 'Classic Delta' distance first proposed by Burrows (2002) was used. To calculate it, values are standardised to standard scores, then the distance between two vectors is defined as the length of the vector of the one minus the other in Euclidean space. Evert et al. (2017) study different distances and find that the cosine distance (defined as the cosine of the angle between the two vectors) tends to outperform the others. Applied to our sample, the differences were minor (results not depicted). 


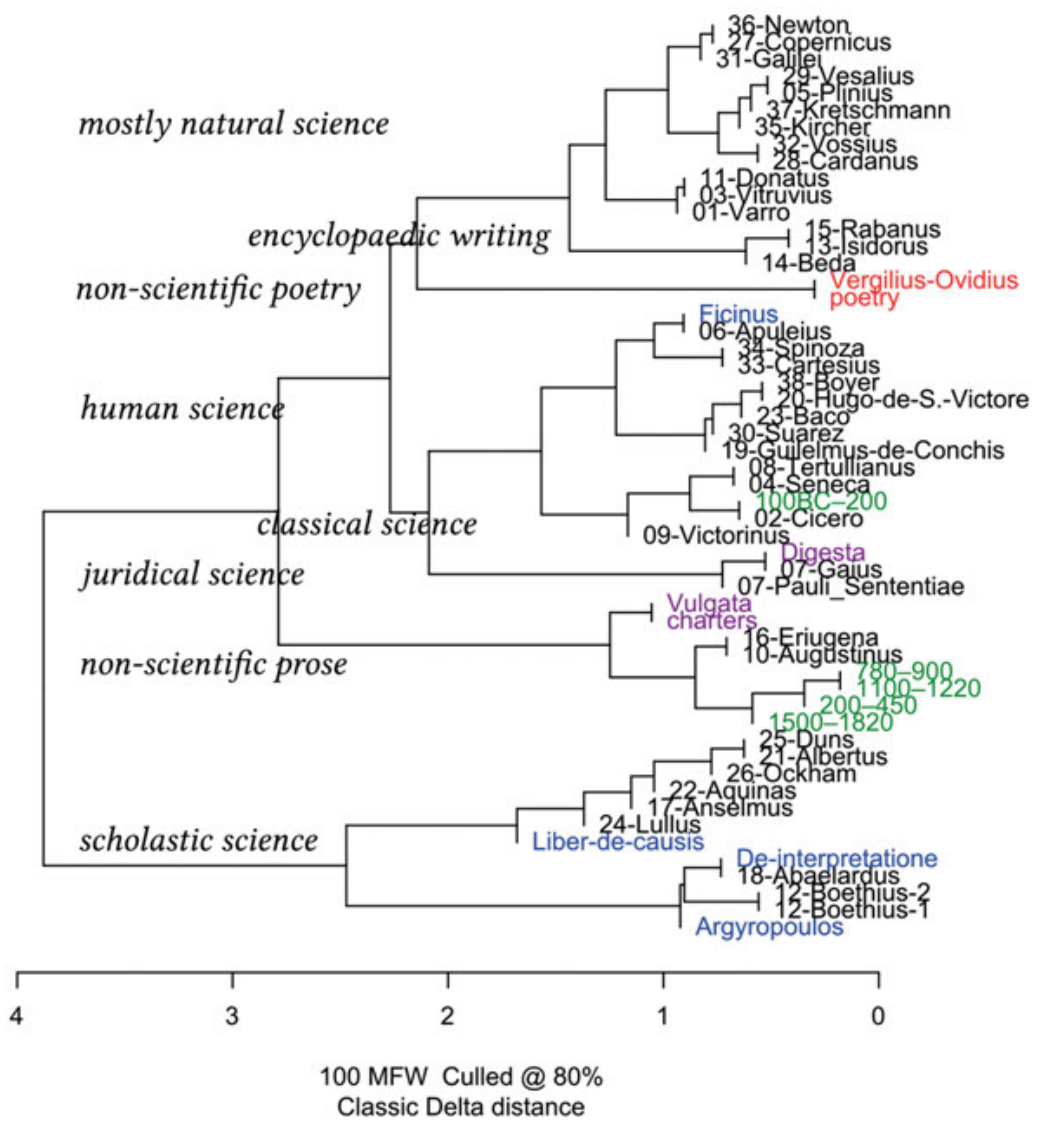

Fig. 39: Stylometric clustering of 100 most frequent words.

stable. In fact, with changing parameters, they tend to shift easily, as do some, apparently not clear, texts. On the whole, these results based on the core vocabulary seem less convincing and stable than those obtained above using grammatical categories and PCA.

A word of caution may be in order: the plots in this chapter are based on very basic values that were determined automatically. They are only as good as the input values. The approach is relatively primitive. If it were possible to automatically generate confident syntactic trees and compare them, the results would become more trustworthy and telling. That said, it is all the more remarkable to see that such basic values as the ones used yield results that are surprisingly close to what a literary critic would assess the Latin types of language of these authors to be. Of course, there are cases that do not square well; they may be interesting in 
making us think about why certain authors end up in different places than expected. Although this approach can quantify differences in scientific (or other) style ${ }^{49}$ it certainly cannot supplant stylistic evaluation by critics, which is based on much more complex 'input' (the reading and understanding of the texts) - but it may enrich it and suggest new ways at looking at it. The next chapter compares the findings with non-computational impressions.

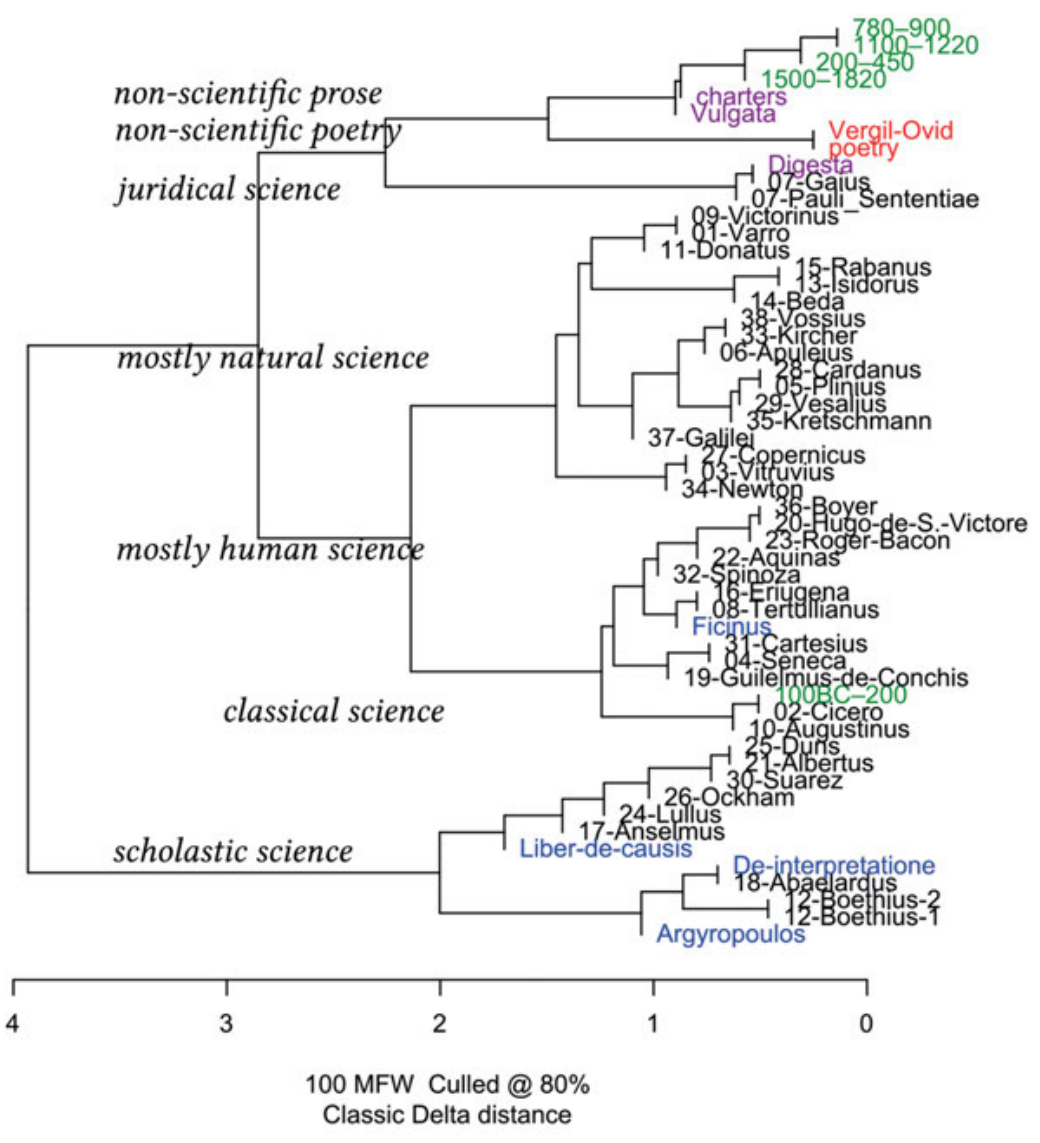

Fig. 40: Stylometric clustering of 100 most frequent lemmata.

49 We use the term 'style' in the following in its most comprehensive sense, as the entire linguistic form not directly related to the content. 